\title{
UNDERSTANDING THE DIGITAL CARDWALL FOR AGILE SOFTWARE DEVELOPMENT
}

\author{
by \\ Stevenson Gossage
}

\author{
A thesis submitted to \\ the Faculty of Graduate Studies and Research \\ in partial fulfillment of \\ the requirements for the degree of \\ MASTER OF COMPUTER SCIENCE
}

School of Computer Science

at

CARLETON UNIVERSITY

Ottawa, Ontario

May, 2014

(C) Copyright by Stevenson Gossage, 2014 


\section{Table of Contents}

List of Tables $\quad$ v

List of Figures

Abstract $\quad$ vii

Acknowledgements viii

$\begin{array}{llr}\text { Chapter } 1 & \text { Introduction } & 1\end{array}$

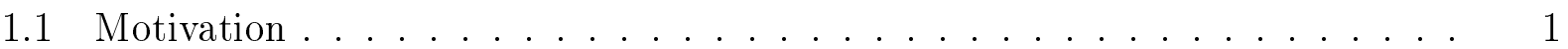

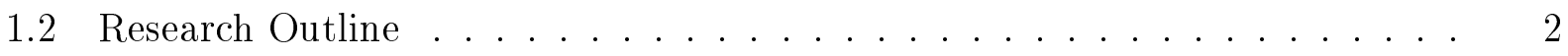

1.3 Thesis Structure . . . . . . . . . . . . . . . 2

$\begin{array}{lll}\text { Chapter } 2 & \text { Background } & 4\end{array}$

2.1 Agile Software Development . . . . . . . . . . . . . . 4

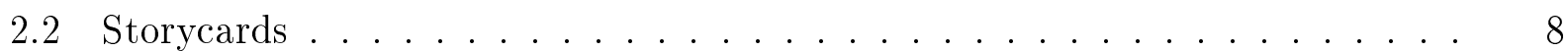

2.3 The Process of Stories . . . . . . . . . . . . . . . 9

2.4 Physical Cardwalls ............................... 11

2.5 Digital Cardwalls and Story Repositories . . . . . . . . . . . 15

2.5.1 Agile Planner for Digital Tabletop . . . . . . . . . . . . 15

2.5.2 Collaborative Multi-touch Agile Planner . . . . . . . . . . 16

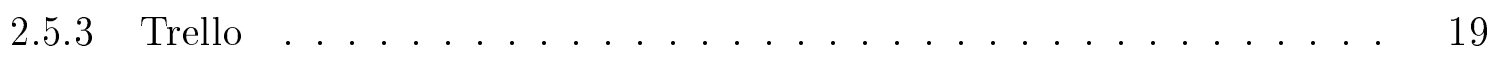

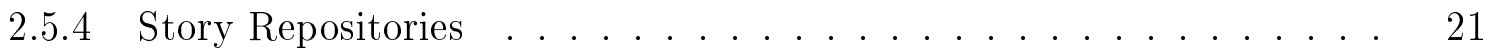

$\begin{array}{lll}\text { Chapter } 3 & \text { The Pilot Study } & 24\end{array}$

$\begin{array}{lll}\text { Chapter } 4 & \text { Field Study of Digital Cardwall Users } & 27\end{array}$

4.1 Motivation . . . . . . . . . . . . . . . . 27

4.2 Research Questions . . . . . . . . . . . . . . . 28

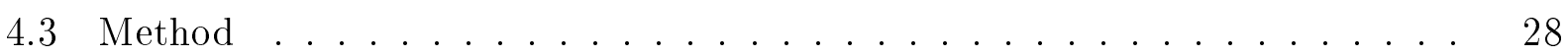


4.3 .1 Participants . . . . . . . . . . . . . . . . . . 28

4.3 .2 Procedure . . . . . . . . . . . . . . . . . . . . . . . . . 29

4.3 .3 Analysis . . . . . . . . . . . . . . . . . . 30

4.3 .4 Ethics Approval . . . . . . . . . . . . . . . . . . . . 31

4.4 Results . . . . . . . . . . . . . . . . . . . . . . 31

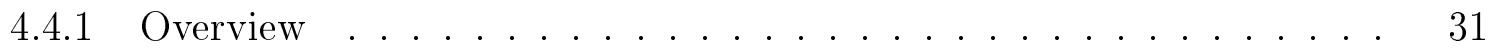

4.4 .2 Themes Identified . . . . . . . . . . . . . . . . 38

4.4 .3 Analysis Bias . . . . . . . . . . . . . . . . 53

4.5 Research Questions Answered . . . . . . . . . . . . . . . . . 53

4.5.1 Distributed Agile Teams . . . . . . . . . . . . . . . 53

4.5.2 Usability of Digital Cardwalls . . . . . . . . . . . . . . 54

4.5 .3 New or Improved Features . . . . . . . . . . . . . . . . . . 54

Chapter $5 \quad$ Implications for Design $\quad 55$

5.1 Digital Cardwalls . . . . . . . . . . . . . . . . . . 55

5.2 Challenges in Presenting Detailed Information . . . . . . . . . . . 57

5.3 Exploring and Filtering Information . . . . . . . . . . . . . . 60

5.4 Managing Backlogs . . . . . . . . . . . . . . . . . . . 61

5.5 Multi-disciplinary use of Stories . . . . . . . . . . . . . . . 64

5.6 Keeping the Cardwall Updated . . . . . . . . . . . . . . . . 66

5.7 Summary . . . . . . . . . . . . . . . . 67

\section{$\begin{array}{lll}\text { Chapter } 6 & \text { Interacting with Surface Technologies } & 72\end{array}$}

6.1 Collaboration for Surface Interactions . . . . . . . . . . . . . . . . 73

6.2 Pointing and selecting $\ldots \ldots \ldots \ldots \ldots \ldots \ldots \ldots$

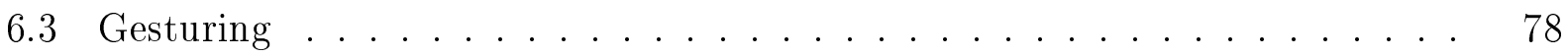

6.4 Hovering . . . . . . . . . . . . . . . . . . . . 83

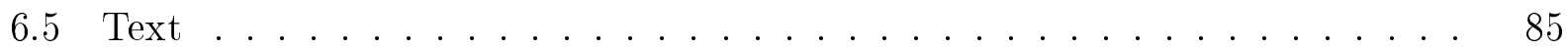

6.6 Identity of the interactor $\ldots \ldots \ldots \ldots \ldots$

6.7 Issues Arising for Digital Cardwalls $\ldots \ldots \ldots . \ldots \ldots$ 
$\begin{array}{lll}\text { Chapter } 7 & \text { Conclusions } & 91\end{array}$

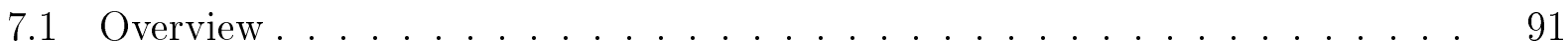

7.2 Discussion . . . . . . . . . . . . . . . . . . . . 91

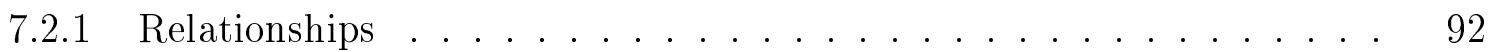

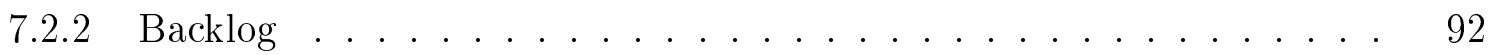

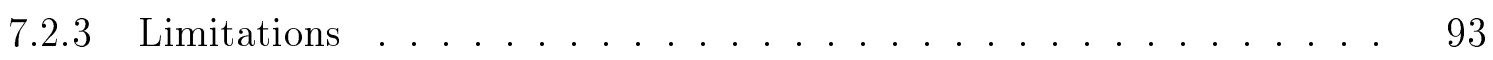

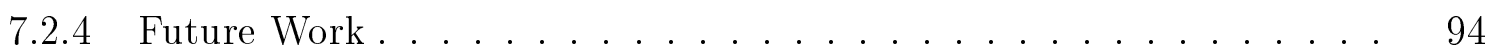

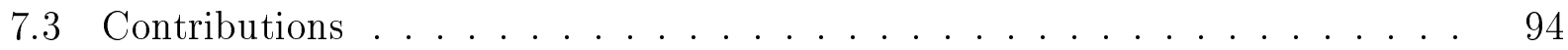

$\begin{array}{ll}\text { Bibliography } & 95\end{array}$ 


\section{List of Tables}

$4.1 \quad$ Organizations Visited . . . . . . . . . . . . . 32

$4.2 \quad$ Project Table . . . . . . . . . . . . . . . 33

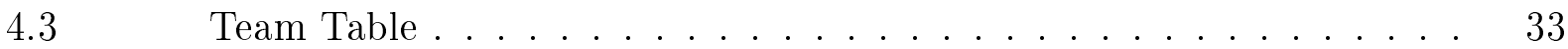

4.4 Demographic Information For Interviewees . . . . . . . . . 36

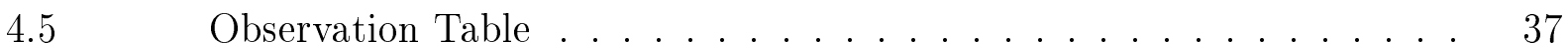

4.6 Interview Table . . . . . . . . . . . . . 37

4.7 Codes used in the analysis of our data grouped by Themes . . . . . 38

5.1 Summary of the guidelines and support from them in Trello and JIRA with JIRA Agile. (F: full support, P: partial support and N: not sup-

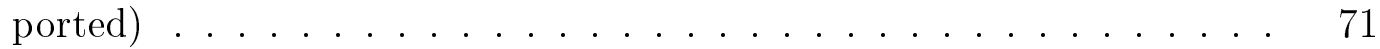




\section{List of Figures}

2.1 Workflow comparison between Waterfall, XP and Scrum [thanks to www.flickr.com user kienntbk1983] . . . . . . . . . . 5

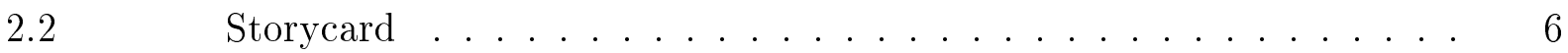

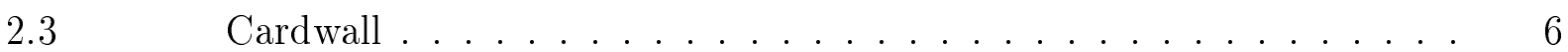

$2.4 \quad$ The Scrum Workflow . . . . . . . . . . . . . . . 11

$2.5 \quad$ Cognitive Dimensions used by Sharp et al. . . . . . . . . . . . 13

$2.6 \quad$ Custom Gestures . . . . . . . . . . . . . . . . . 17

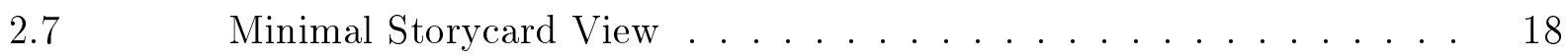

$2.8 \quad$ Full Storycard View . . . . . . . . . . . . . . . 18

$2.9 \quad$ A Trello Cardwall for Household events . . . . . . . . . . . . . 20

$2.10 \quad$ A Trello Agile Cardwall . . . . . . . . . . . . 20

$2.11 \quad$ Greenhopper Cardwall Plugin for JIRA . . . . . . . . . . 22

$2.12 \quad$ A typical JIRA Cardwall . . . . . . . . . . . . . . . 23

$4.1 \quad$ A typical JIRA Cardwall . . . . . . . . . . . . . 34

$4.2 \quad$ A story from the StoryBoard Digital Cardwall . . . . . . . . . . 35

$4.3 \quad$ An example of the StoryBoard Digital Cardwall . . . . . . . . . 35

6.1 Selecting high resolution items with a low resolution pointing device . $\quad 77$

$6.2 \quad$ FingerGlass: a widget for interacting with large displays . . . . . 78

$6.3 \quad$ Wobbrock et al.'s Taxonomy of Surface Gestures . . . . . . . . . 79

$6.4 \quad$ Hovering in web applications . . . . . . . . . . . . . 82

$6.5 \quad$ Hovering and Google Search . . . . . . . . . . . . 82

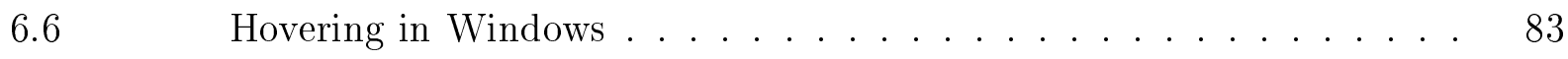

$6.7 \quad$ A solution to hovering on large displays . . . . . . . . 85

$6.8 \quad$ A simple QWERTY virtual keyboard . . . . . . . . . . 86

6.9 Text input on large displays . . . . . . . . . . . 87

$6.10 \quad$ A few examples of how IdLenses can be used . . . . . . . . . . 89 


\begin{abstract}
In Agile software development, key artefacts used to support the process are the User Story (usually recorded on a Storycard) and Story Cardwall (usually a dedicated portion of a wall). These low-fidelity tools work together to help teams stay focused and self-manage their projects. The need to support distributed teams and team members makes the physical Cardwall impractical and teams are therefore migrating towards digital story management tools. We wanted to learn how to design a digital Cardwall that leverages the benefits of the physical Cardwall, while adding more value with features only possible in a software system. We conducted field studies of Agile teams and performed qualitative data analysis to understand the needs for digital Cardwalls. We then used these findings to identify guidelines for future design.
\end{abstract}




\section{Acknowledgements}

I'd like to thank my family for all their support and especially my wife Lucy who has put up with endless late nights and early mornings.

I'd also like to thank my colleagues in the Hotsoft lab who are always available and willing to help.

I'd like to thank Dr. Sonia Chiasson and Dr. Timothy Lethbridge, members of my thesis examination committee. Their insightful comments, fair questions and feedback helped improve the final version of this thesis.

A special thanks to my supervisor, Dr. Robert Biddle who encouraged me to start on this academic path and who never lost faith even when things were not progressing as expected.

Finally, I'd like to thank Dr. Judith Brown, a friend and colleague, with whom I have worked in close collaboration for the past few years. Judith has played a key role in keeping me focused and on track. Without her positive motivation and support I would not have arrived at this point. 


\section{Chapter 1}

\section{Introduction}

\subsection{Motivation}

Agile methodologies promise increased productivity for software development teams by providing proven strategies, guidance and best practices. Agile software development teams work iteratively with frequent software releases, which allows their customers to provide frequent input and feedback, ensuring the direction of the project is in-line with customer needs. The customers' other key role helps to drive product development by selecting key features for developers to implement in the next iteration. At the root of the Agile tool-set, is the User Story (usually recorded on a Storycard) and Story Cardwall (usually a dedicated portion of a wall). These low-fidelity tools work together to help teams stay focused and self-manage their projects. The Cardwall helps to foster awareness and encourages transparency by acting as an information radiator, where the User Stories are on display and stakeholders can easily track the progress of the development work.

User Stories are the fundamental unit of work, they are written from the user's perspective, by the customer and intended to represent some functionality that a specific user would need while interacting with the system. The story is role-based, high-level, and end-user oriented. The story is a small, but important, part of the system under development and is intended to encourage better communication with the customer. To this end, the story should be brief, with just enough detail for the developer to understand the big picture, but with enough information that the remaining details could easily come out via further conversations with the customer. Similar to the constant customer feedback possible with frequent releases, the quality and direction of the current software being implemented is guided by the constant conversations required between developer and client. These conversations help ensure that the current iteration will produce working software that meets the customers' expectations.

Traditionally, the Story Cardwall is on constant display in a public space preferably close to where the developers work. The Cardwall will typically have columns, representing 
the team's workflow and the Storycards are moved from one column to the next as their implementation progresses. In this way, the Cardwall allows the developers and any interested party to quickly understand the state of the current iteration. Physical Storycards and Cardwalls are very low-tech, and often made using sticky-notes for the Storycards; these are ideal since they can be stuck to many surfaces. The physical Cardwall is just as simple, and often, simply occupies empty space on a wall near the developers. The columns and column labels of the Cardwall are made visually distinct using whatever may be available, including masking tape for the columns and larger sticky-notes or simple paper for the labels. Even now, the physical Cardwall is still the recommended tool for Agile teams; however, the need to support distributed teams and team members makes the physical Cardwall impractical and teams are therefore migrating towards digital story management tools. This move toward the digital Cardwall is at the root of the motivation for this thesis. We want to learn how digital Cardwalls are used in practice by professional Agile teams, with a particular focus on understanding user interactions to inform the development of design guidelines for creating better digital Cardwalls.

\subsection{Research Outline}

Our goal was to understand how to design a digital Cardwall. To accomplish this we looked at the advantages of physical Cardwalls, and reviewed the state of the art in story management tools and digital Cardwall applications that leverage multi-touch technology. We then conducted a pilot study followed by field studies, including interviews and observations of software development teams. We adopted a qualitative approach and used thematic analysis to help understand our data and interpret our findings. We used these findings to identify a set of guidelines for the interaction design of digital Cardwalls. Lastly, we reviewed the principles of interaction design for large multi-touch displays, and discussed how multi-touch could be used to further enhance the usability of a digital Cardwall.

\subsection{Thesis Structure}

The remainder of the thesis is structured as follows. 
Chapter 2: We present relevant background work. We begin with an outline of Agile software development and the importance of User Stories. Traditional physical Cardwalls are discussed including their advantages. We then outline recent work on multi-touch digital Cardwalls and story and issue trackers.

Chapter 3: We explain the details of our pilot study of a real world Agile development team and how it informed the design of our field study.

Chapter 4: We present the details of our Digital Cardwall Study including the motivation, and its design. We describe the laborious task of coding the study data and we describe how we leveraged thematic analysis to help us analyse our data with the goal of understanding future design requirements. The results are presented and the chapter finishes with a discussion of each of the identified themes.

Chapter 5: We discuss design implications based on the findings from Chapter 4. We identify a set of design guidelines as a result.

Chapter 6: We present a survey of common interaction issues when using large surfaces and multi-touch technology and how these could be applied to enhance the usability of a digital Cardwall.

Chapter 7: We present a review of our work, identify our contributions and outline future work. 


\section{Chapter 2}

\section{Background}

\subsection{Agile Software Development}

Today, many lightweight Agile processes have emerged, however, for the purpose of this thesis we will consider Extreme Programming (XP) [8] and Scrum [57], the two most common Agile methodologies. Both of these methodologies take an iterative approach where less focus is put on upfront analysis and more on quick consistent deployment of quality working software. XP uses the term 'iteration', and it is common to emphasize that these be short; as little as one week is common. Scrum uses the term 'sprint' which tend to last longer, about a month. In Extreme Programming, the idea is to deliver working software to the customer at the end of each iteration. Similarly with Scrum, the goal is always to have each sprint produce working software; however, it is not always released to the customer. Nevertheless, both terms (iterations and sprints) are commonly used in the Agile community and often used interchangeably. For the remainder of this paper we will use the term iteration broadly to refer to the general idea of a short time-boxed release cycle. Figure 2.1 compares a 2year project using the traditional waterfall approach to development versus XP and Scrum. Notice the biggest difference is the number of deliverables.

There are a variety of distinct roles that are important to the success of an Agile team. The roles vary between XP and and Scrum and will be described separately.

In XP [8], the typical roles are the 'customer', the 'developer', and the 'coach'. The customer is responsible for the creation of User Stories and the functional tests which help define when stories are done. The customer is also responsible for explaining and providing the developers with the details needed to successfully implement a story. The developer role is usually played by three to seven developers and their primary responsibility is to implement and test the functionality that the story describes. They are also responsible for estimating the stories and they should be in contact with the customer throughout the implementation of the stories to ensure that their implementations align with the customer's expectations. 


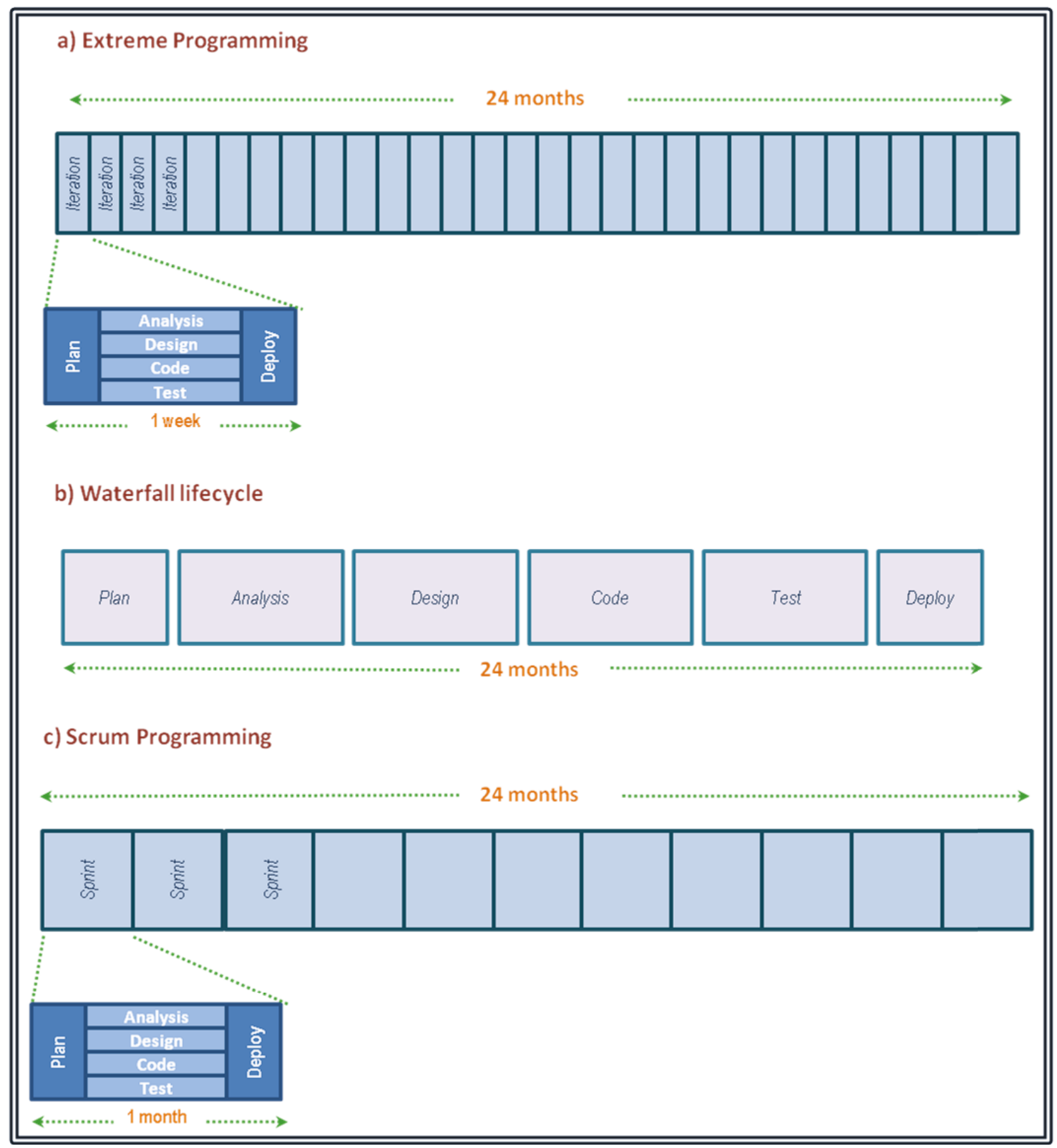

Figure 2.1: Workflow comparison between Waterfall, XP and Scrum [thanks to www.flickr.com user kienntbk1983] 
As a for um user

I can soe posters'

profile pictures as avatars

Figure 2.2: Storycard [thanks to www.flickr.com user J'Roo's]

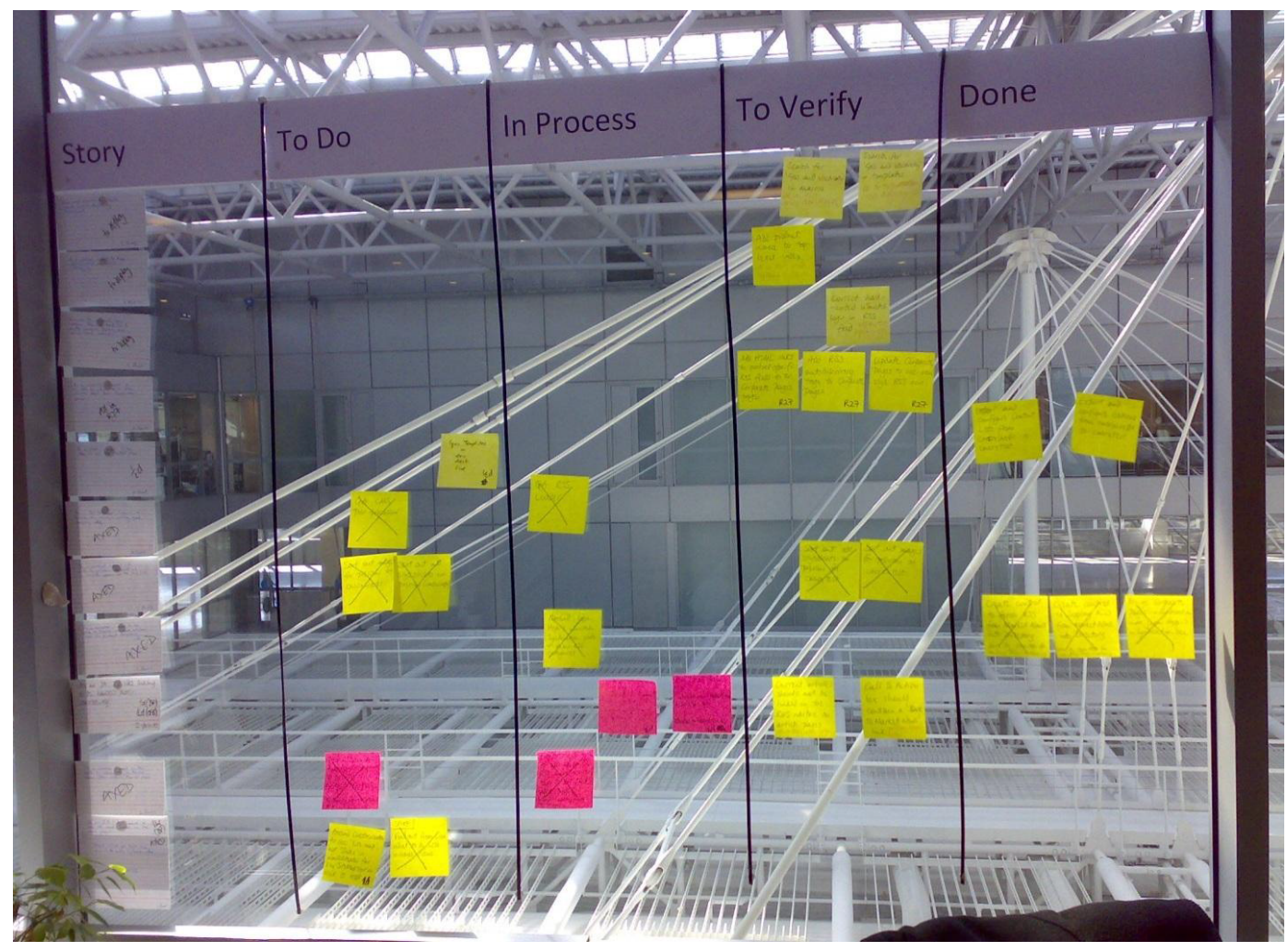

Figure 2.3: Cardwall [thanks to flickr.com user owenblacker] 
The coach is responsible for ensuring that the practices of XP are followed and is available as a mentor to the team to instil the principles and values of XP.

In Scrum [57], the main roles are the 'product owner', the 'developer', and the 'Scrum Master'. The product owner is in charge of the backlog and is responsible for the creation of stories, their prioritization and scheduling. The developer role is basically the same as in $\mathrm{XP}$, although there are fewer technical practices, such as testing, that are specified by Scrum. The Scrum master, like the XP coach, is responsible for helping to manage the process.

The Storycard and Cardwall are physical artefacts that help Agile practitioners especially during planning and the active development phases of a software product's life cycle. User Stories are one of the most common lightweight artefacts used by many Agile methodologies. When used in conjunction with the story Cardwall as recommended in both XP and Scrum, Agile practitioners are able to increase awareness and transparency, engage the customer, and develop better software that meets the expectations of the customer with fewer errors, in a shorter time frame. The Storycard (see Figure 2.2) and the Cardwall (see Figure 2.3) are artefacts that complement each other. The Storycard helps capture requirements in the form of User Stories, and the Cardwall helps track the progress of those stories during a single iteration in a very public and transparent way. An important side-effect of using the Cardwall as recommended is that it acts as a catalyst for communication within the development team members, but also between the team and their customers and other stakeholders. Initial discussions between the customer and the developers begin when stories are first created. The conversations continue as the stories are prioritized and scheduled. Finally it is the discussions that draw out the details of the stories while they are in active development. The Cardwall, on the other hand, acts as an Information Radiator [15] and is invaluable in terms of the information that can be gathered at a glance. The Cardwall quickly reveals whether or not an iteration is on schedule or falling behind. As the Storycards move between the columns of the Cardwall, the team and project stakeholders can easily understand the state of those stories; as the columns align with a team's workflow. The Cardwall is typically organized in a manner to show work completed, work in progress, bugs, and work yet to be scheduled. 


\subsection{Storycards}

The traditional Storycard is a physical artefact usually similar in size to an index card, postcard or even a sticky-note. Customers use Storycards to record User Stories which describe some functionality that needs to be implemented in the software. The language of User Stories is customer-centric, which is ideal since the customer is close to the product and they know the daily routines of how the tasks are currently accomplished. This allows the customer to describe what really needs to happen at a high level since they are not expected to know the language of the developer. Their expertise comes from the customers' domain and their viewpoint is through their interaction with the software via its user interface. Davies explains that the size of the Storycard is deliberately small, preventing unnecessary verbose detail, "The Card may be the most visible manifestation of a User Story, but it is not the most important [20]". Davies continues to explain that cards "represent customer requirements rather than document them." This emphasises that the actual text on the card is simply a reminder or placeholder while "the details are worked out in the Conversation and recorded in the Confirmation [17]." In the process of creating stories, the following advice from Jeffries should be considered.

"Words, especially when written, are a very thin medium through which to express requirements for something as complex as software. With their ability to be misinterpreted we need to replace written words with frequent conversations between developers, customers, and users. User Stories provide us with a way of having just enough written down that we don't forget and that we can estimate and plan while also encouraging this time of communication [17]."

Jeffries describes the procedure as the combination of the three 'C's: Card, Conversation and Confirmation [35]. The process starts with a developer or pair of developers choosing a story to work on; "the physical possession of this card by a developer is a warrant that secures the conversation (and the confirmation process of the acceptance test) with a customer [60]." The subsequent communication between developer and customer explores the details of the story. The confirmation should be mutually agreed upon, such that the story's completion is well understood. 


\subsection{The Process of Stories}

User Stories are the essential artefact in the agile workflow. User Stories are used similarly by Agile teams, although there are many variations in the details of their usage. A project typically starts with the creation of a project backlog (a collection of User Stories), which helps define the scope of the project. Ideally, to get a good 'big picture' overview of the project and to get a sense of its scope, as many stories as possible will be created in an attempt to capture all of the high-level system requirements. Often the initial product backlog is mostly a collection of "epics", which are User Stories with a very high level of abstraction. During planning, the epic User Stories are discussed with the customer and broken down into smaller User Stories which collectively capture the requirements of the epic. Although it is recommended that the backlog be as complete as possible at the start of a project, for business reasons it is often the case that development work must start in parallel to the creation of the backlog. In this case, the backlog might only start with enough stories for an iteration or two. Generally, the physical representation of a backlog is a stack of cards on a table or a collection of sticky-notes on a wall.

Prioritization Once the stories are created, the backlog must be prioritized at least in part. To do this, the customer, in conjunction with the team, decides which stories should be implemented first. The prioritization process is flexible and the extent to which teams prioritize their backlog can differ across distinct teams; however it can range from planning the stories for the next iteration to planning for several iterations. As iterations are planned, if the desired functionality is captured by an epic Storycard, then it will typically be discussed carefully with the customer and an agreement should be made about how to decompose the epic into smaller scoped stories. This is especially critical when planning the next iteration so that epics are not scheduled to be worked on before they are ready.

Estimation The estimation process follows and normally only apply to the stories that have been selected for the next iteration. To estimate, developers discuss the story and decide how long they believe it would take to implement. Often it is during the estimation process where epics are identified and broken down into smaller User Stories. The ideal scope for any individual User Story would be when the estimate for the story is less than or equal 
to the duration of an iteration. If the estimate indicates that the story will be longer then an iteration then the team may choose to break it down into smaller stories which would be estimated individually. Often when this happens, some of the resulting stories can be moved out of scope of the next iteration; other times, the team may choose not to break it up and allow it to span multiple iterations (if other stories do not depend on it being implemented). The higher the level of abstraction of a User Story, the more conversation must be involved to work out the details. At the same time, the flexibility in using higher level User Stories helps to reduce a team's cognitive load by allowing teams to concern themselves with the details at the point when they are needed.

Releases Every iteration results in the release of completed functionality to the customer and this is generally regarded as a release. It is also common for teams to use major and minor releases. A major release would involve a significant incremented in the product version. For example, a major release might mean going from version 1.1.0.3 to 1.2.0.0 or maybe from 1.9 to 2.0, whereas a minor release would be an increment of the right-most digit of their product version. A major release marks a milestone and represents the completion of a significant portion of the product. Each iteration is composed of planning, development, testing and a retrospective. As previously mentioned, this typically lasts from one week to one month.

The planning phase of an iteration includes the prioritization and estimation of stories for the purpose of selecting the ones that are most important for the current stage of the project. In the development and testing phase, the stories are put into active development and are implemented and tested. It is in this phase where the communication between developers and customers is critical so that the details are understood and the implementations are correct according to the customers' expectations. Finally, the retrospective is where the process is gauged and analysed and a general determination as to the overall health of the project is made. The intent of the retrospective is to constructively identify and attempt to correct any problems within the team, process or even with regards to communication within the team or with the customer. It is a chance to learn from mistakes and correct them before they become a permanent part of the process.

Figure 2.4 shows the basic Scrum workflow from a collection of stories in the product backlog, to the selection of the most important stories for the sprint backlog, to the actual development cycle or sprint and finally to the working piece of software which may or may not 


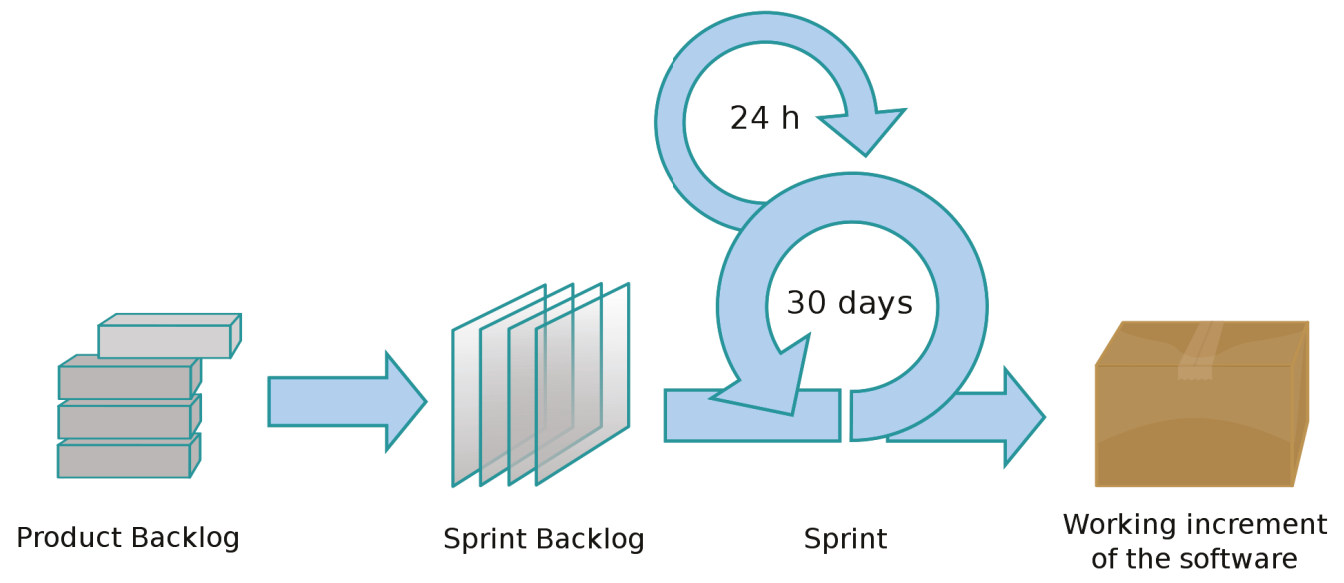

Figure 2.4: The Scrum Workflow [http://commons.wikimedia.org/wiki/File:Scrum_ process.svg]

be delivered to the customer. This process is essentially repeated until the last sprint is done and the product backlog is empty. The workflow in XP is similar, however, the iterations are typically shorter and there is only one backlog.

\subsection{Physical Cardwalls}

The Cardwall is a physical artefact that is typically used as a tool for planning and tracking the progress of an iteration during the active development of a software product.

A natural question might be to wonder how this level of detail and complexity can be captured on a few cue cards pinned to a wall, and, maybe more importantly, how does this Cardwall actually help software development teams to meet their goals and deadlines while producing quality code? Often it is the human component that is the key to the success of the method. Any software trying to support a human task must involve in-depth consideration of this issue. The software must provide an environment which supports and encourages interactions which bring success to the human task. As Sharp et al. say, "Any Agile team looking to move towards digitising the team's support will need to take account of the complex relationships that exist within this social system if they wish to retain key properties of successful teams [60]." 
Sharp et al. conducted an in-depth five-year observational study of XP software development teams using Storycards and physical Cardwalls and the results were published in several papers. The following is a summary of one of those papers, "The role of physical artefacts in Agile software development: Two complementary perspectives" [60] which was in large part inspiration for the work in this thesis. This paper investigates a team's use of Storycards and Cardwalls in terms of physical interactions and social interactions. The findings carry severe implications which need to be considered for the successful design and implementation of a digital Cardwall. The authors looked at the use of both Storycards and Cardwalls from the perspective of notation and applied Green's Cognitive Dimensions framework [28] to understand the importance of each notation and how it supports the intended activities in terms of the different cognitive dimensions. The specific cognitive dimensions used in the analysis of their work are outlined in Figure 2.5. The investigation also focused on social context and how the social nature of the process binds and supports the use of Storycards and Cardwalls. It also addresses how the social context frames the underlying agreements about how these artefacts are used to ultimately support the goal of producing working software.

In general, Sharp et al. found that the Storycard notation generally supported the following cognitive dimensions: abstraction, closeness of mapping, low diffuseness, provisionality, and low viscosity. User Stories capture requirements and are therefore an abstraction of them, which also means they are necessarily close to the domain which supports closeness of mapping. Low diffuseness is supported by the stories being written in the language of the user, that they are brief and terse by design because they are only intended to be a reminder for further discussion. The Storycard medium on which the story is presented, i.e., on an an index card or sticky note, gives the Storycard a feeling of provisionality. This also supports low viscosity because it encourages the engagement of a Storycard by the users of the Cardwall. The Storycard does not have much support for the following dimensions, error proneness, progressive evaluation, premature commitment, hidden dependencies, and hard mental operations.

The Cardwall generally supported the following dimensions: provisionality, low viscosity and process visibility. It is easy to move cards, change labels and, start new iterations which all contribute the Cardwall's high provisionality and low viscosity. Also, the Cardwall's columns help reveal the underlying process and can be easily understood, which makes the 


\begin{tabular}{|c|c|}
\hline $\begin{array}{l}\text { Cognitive } \\
\text { dimension } \\
\end{array}$ & Definition \\
\hline Abstraction & Can elements be encapsulated? If so, to what extent? \\
\hline $\begin{array}{l}\text { Closeness of } \\
\text { mapping }\end{array}$ & $\begin{array}{l}\text { How directly can the entities in the domain be expressed in the } \\
\text { notation? Does the notation include entities that match the } \\
\text { key concepts or components of the domain? }\end{array}$ \\
\hline Consistency & $\begin{array}{l}\text { When some of the language has been learned, how much of } \\
\text { the rest can be inferred? Are similar features of structure and } \\
\text { syntax used in the same way throughout? }\end{array}$ \\
\hline Diffuseness & $\begin{array}{l}\text { How many symbols or graphic entities are required to express } \\
\text { a meaning? }\end{array}$ \\
\hline Error-proneness & Does the design of the notation induce 'careless mistakes'? \\
\hline $\begin{array}{l}\text { Hard mental } \\
\text { operations }\end{array}$ & $\begin{array}{l}\text { Does the notation use mechanisms such as nesting and } \\
\text { indirection that require mental unpacking or 'decoding'? For } \\
\text { example, are there places where the user needs to resort to } \\
\text { fingers or additional annotation to keep track of what's } \\
\text { happening? }\end{array}$ \\
\hline $\begin{array}{l}\text { Hidden } \\
\text { dependencies }\end{array}$ & $\begin{array}{l}\text { Is every dependency overtly indicated in both directions? Is } \\
\text { the indication perceptual or only symbolic? }\end{array}$ \\
\hline $\begin{array}{l}\text { Premature } \\
\text { commitment }\end{array}$ & $\begin{array}{l}\text { Do developers have to make decisions before they have the } \\
\text { information they need? }\end{array}$ \\
\hline $\begin{array}{l}\text { Progressive } \\
\text { evaluation }\end{array}$ & $\begin{array}{l}\text { Can a partially-complete representation be executed or } \\
\text { evaluated to obtain feedback on 'how am I doing?' }\end{array}$ \\
\hline Provisionality & Can indecision or options be expressed? \\
\hline $\begin{array}{l}\text { Role- } \\
\text { expressiveness }\end{array}$ & $\begin{array}{l}\text { Can the reader see how each component relates to the whole, } \\
\text { and what the relationships between notational elements are? }\end{array}$ \\
\hline $\begin{array}{l}\text { Secondary } \\
\text { notation }\end{array}$ & $\begin{array}{l}\text { Can developers use layout, colour and other cues to convey } \\
\text { extra meaning, above and beyond the 'official' semantics of the } \\
\text { language? }\end{array}$ \\
\hline Viscosity & $\begin{array}{l}\text { How much effort is required to perform a single change? How } \\
\text { much effort is required to perform multiple changes of the } \\
\text { same type? Does making one change then have the 'knock on' } \\
\text { effect of requiring other changes? }\end{array}$ \\
\hline Visibility & $\begin{array}{l}\text { Is every part of the notation simultaneously visible - or is it at } \\
\text { least possible to juxtapose any two parts side-by-side at will? } \\
\text { If the notation is dispersed, is it at least possible to know in } \\
\text { what order to read it? }\end{array}$ \\
\hline
\end{tabular}

Figure 2.5: Cognitive Dimensions used by Sharp et al. 
visibility of the process high. There were also dimensions that were not directly supported by Cardwalls: consistency, hidden dependencies, role expressiveness, progressive evaluation and error-proneness.

The Storycard or Cardwall notation alone did not directly support all of the dimensions. Social agreements, discipline and interactions that framed the use of the Storycards and Cardwall and contributed to the support of the missing cognitive dimensions. Without the social interactions the benefits of the Storycard and Cardwall would be significantly reduced.

While a general template for stories usually exists such that key information is presented as follows: "As A $<$ Role >", "I want $<$ Description >", and "So that $<$ Benefit $>$ ". The use of Storycards is extremely flexible and we commonly find differing notations being used across Agile teams. At the same time, within any one team the notation and use of cards is strictly adhered to. Everything on the card has meaning which is not necessarily clear to an observer unfamiliar with the team or their specific notation. The location of the Storycard, its color, the size of the lettering and any other annotations carry significant meaning and thus provide a high level of abstraction. A mature established team might have a well-defined notation while a new team might still be looking for what works best for them, but the beauty of the physical cards is that it doesn't restrict a team to any one notation; and teams are free to find their own ideal solution.

The use of the Cardwall is also an extremely flexible procedure but has its generalities in that teams use walls for the duration of a project and leave them on constant display where they are easily seen - usually in a common space where anyone walking by could get an idea of the progress of the team. The Cardwall is generally regarded as an 'Information Radiator' [15] and helps ensure the transparency of the project. The Cardwall is full of meaning not obvious to an observer who lacks familiarity with Agile methodologies or team specific notation. However, key information that one should be able to gather almost instantaneously from any Cardwall is the general progress of the project. The placement of stories signifies whether or not they are in active development, waiting to be started, finished, etc. Again the Cardwall is generally without structure when considering its use among distinct teams, but it is used in an extremely consistent manner within any one team.

Using Cardwalls requires an active participation between customers and developers and was originally described as 'The Planning Game' [8], where the objective basically would be 
to prioritize the cards, sort them into releases and sprints, assign them to developers and have the developers accept or decline them until the cards were sufficiently sorted into at least the next sprint; unassigned stories are left in the project backlog, to be dealt with at the next iteration or release planning meeting.

The social interactions involved in the whole process enable teams to determine their best use of the Storycard and Cardwall's notation, which includes the organization and layout of the Storycards on the Cardwall. These interactions reveal the importance and meaning of the stories and thus drive their physical placement on the Cardwall, which, in turn drives the story's progress through the system. The physical size of the cards is also of utmost importance since it limits the information it can hold, and therefore encourages a communicative dialog.

\subsection{Digital Cardwalls and Story Repositories}

Large high-resolution displays are now readily available, as is the support for multi-touch capabilities. Leveraging these technologies seems like an obvious place to start when thinking about developing a digital Cardwall. Every day more devices are being produced at a reasonable cost with support for two or more simultaneous touches; a critical feature for the development of truly collaborative tools.

\subsubsection{Agile Planner for Digital Tabletop}

The best example of similar software is the Agile Planner for Digital Tabletop (APDT) [66]. APDT was designed based on a prototype by Weber et al. [67] which was intended for colocated collaboration on a single touch surface. APDT chose to use this as a starting point, but wanted to enhance it with support for multi-touch, the ability to interface their Cardwall with other Agile planning tools, and real-world evaluation based on user studies. It was designed after observing traditional Agile planning meetings, as well as meetings conducted using the Distributed AgilePlanner (DAP) [46]. As the name suggests DAP was designed to support distributed Agile teams in the planning and maintenance of an Agile project through the use of a digital whiteboard and Storycards. DAP had been developed with a traditional

single user interface paradigm (one keyboard, one mouse), to enable users to collaborate remotely; it did not support multi-user interactions in a co-located environment. APDT also 
studied and drew from the literature available on the use of multi-touch tabletops for group collaboration. APDT was developed as a multi-touch enabled tool, specifically for two tables designed by Smart Technologies Ltd. using Smart's proprietary SDK. The first table used DViT (Digital Vision Touch) [22] technology and had support for two concurrent touches. The second table used FTIR (Frustrated Total Internal Reflection) [27] technology and had support for 40 concurrent touches. The two touch capabilities of the DViT table limited the users' ability to work concurrently, while the small form factor of the FTIR table meant that it was difficult to leverage its support for 40 simultaneous touches.

\subsubsection{Collaborative Multi-touch Agile Planner}

Our own previous work includes Collaborative Multi-touch Agile Planner(CMAP). ${ }^{1}$ APDT (as described above) was a highly functional, full featured tool, however, it was dependent on the Smart SDK and Smart's hardware; a limitation that influenced our design. With CMAP hardware and operating system independence was a goal. We also wanted to support multiple concurrent touches. CMAP extends previous work on Agile tool support by addressing key issues outlined by Scott et al. about group collaboration around digital tabletops [58]. We also attempted to address issues found by Sharp et al. about how the physical nature of Storycards and Cardwalls affect their use and have a "reflexive relationship" with the social interactions in which the use of the Cardwall is grounded [60]. Sharp and her colleagues stress that software designed to digitise the Storycard and Cardwall must carefully consider both the notational and social aspects of these artefacts.

The basic goals for the development of CMAP was to design a digital Cardwall (in fact a horizontal card table) with support for multiple concurrent inputs (to support multiple users), and was not limited to any specific hardware. We wanted to explore the use of gestures and how they could be used efficiently to manipulate the stories. At the time, we were working with horizontal surfaces so we needed to understand the implications of user orientation and their effects on the usability of the tool. We also wanted to create a distributed system with a back-end that could be accessed simultaneously from multiple sites.

To support these goals several design decisions were made, including:

\footnotetext{
${ }^{1}$ Honours Project, Bachelor of Computer Science, Carleton University, 2011.
} 
- The use of PyMT and Python meant built-in platform independence, multiple concurrent user interaction and support for multiple inputs. PyMT allowed the use of gestures and rotatable widgets to deal with orientation issues. Furthermore, since both Python and PyMT are open source, we had the access and the freedom to change the code base when we found the need.

- The need to support persistent and shared data was achieved by using a combination of open source projects including Git, GitHub and the Petaapan Google Application Server. Git took care of local and remote storage while the Google App Server took care of change notification through a publish/subscribe mechanism and notification hook between it and the GitHub repository.

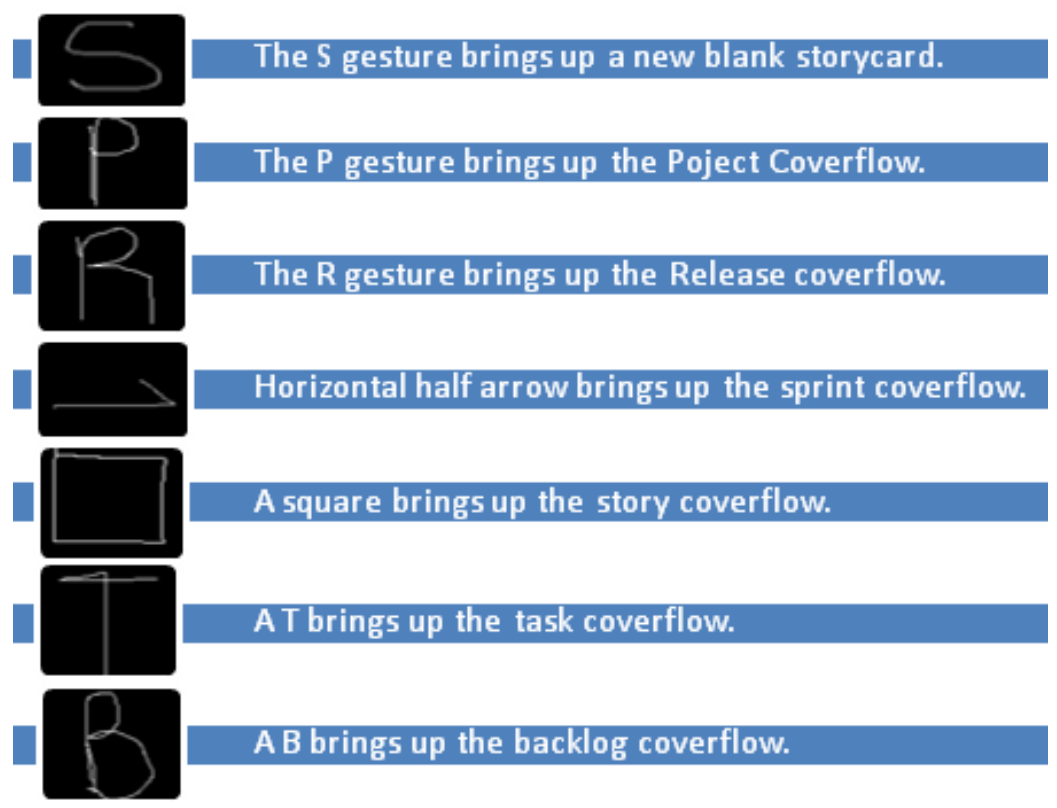

Figure 2.6: Custom Gestures

The use of gestures was an important feature and a unique gesture was provided as a shortcut to each of the most important activities allowed by CMAP. Figure 2.6 displays a list of the custom gestures and a brief description of the functionality of each. A tool had to be developed so that users could customize and create their own gestures much like teams develop and use distinct notations and layouts.

CMAP took a structured approach to the Storycard and Cardwall. This was an attempt to capture all the relevant data that seemed to be critical to the Agile planning process. 
XML was used to define the data of the Storycards and other artefacts and the view was a form-based widget with labels and text fields. Users could use traditional data entry via real or virtual keyboards. In an attempt to limit the amount of information contained by an artefact, a default minimal view was created, which only allowed the entry of a name for the artefact and a description. Figure 2.7 shows the minimal artefact view. A second view was created for stories to allow the user to enter more information. This view was built so that the developer could, in conversation with the customer, elaborate on the information provided by the minimal view. Figure 2.8 shows this more detailed view of a Storycard.

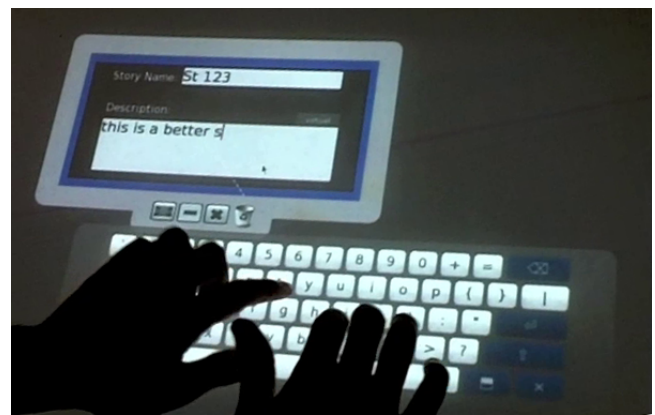

Figure 2.7: Minimal Storycard View

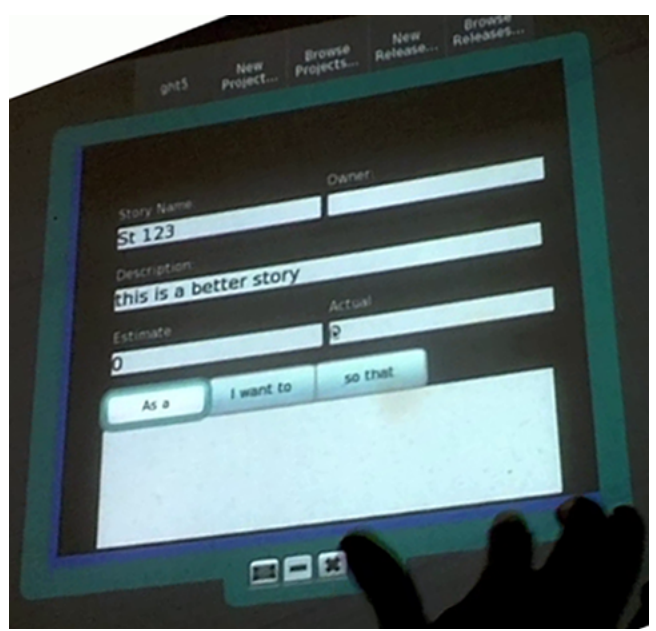

Figure 2.8: Full Storycard View

This structured approach seemed like a good way to capture the data and have it in a format that could easily be stored, shared and adapted to allow it to interface with other software tools like task management systems. This approach could give developers access to the stories directly from their IDE.

To start using CMAP, the user first creates a project. Once created the next step is to create one or more releases, then one or more sprints, then one or more stories and finally, if they choose to, the users could create one or more tasks. The only flexibility in this process is the ability to create stories at any point which are then placed into the backlog. Coverflow widgets were designed to hold artefacts of the same type. These containers are initially empty, except for the ability to create a new artefact of that type. So, to create a project, the user could draw a $\mathrm{P}$ gesture to bring up the project container. From the container, clicking on the New Project button would bring up a new empty project artefact. This process makes the newly created project active such that a new release would automatically become a child of 
this project. This introduces the concept of the current artefact which is intended to control the hierarchical structure of the artefacts. For each artefact type, there could be a current artefact of that type. This allows the system to create the parent-child relationships of the artefacts automatically. For example, once a project is created, it becomes active and will automatically become the parent of all newly created artefacts. If however, a second new project is created, it would then become the new current project, and subsequent artefacts would become the children of this new project. To bring up the releases container, one would use the capital R gesture. Again there is a button to create a new release. In a similar fashion the user can use the gestures outlined in Figure 2.6 to bring up the other containers, and create artefacts of those types. The capital $S$ gesture was reserved as a shortcut to create a new story. As each artefact is created it, becomes active and new artefacts either become siblings or children of the current artefact; in the case of siblings, they inherit their type from the current artefact. For example, a user could go through the process up to the point where they have created a sprint, after which the creation of many stories may be desirable, and as each sibling story is created, it becomes the current artefact, allowing the optional creation of tasks for that story.

In the course of development of CMAP, the direction that was originally taken came into question for various reasons. Two important questions arose as a result of demonstration and consultations with expert practitioners and researchers. The first was a question of flexibility of user interaction with the system and the second was how the rigidity of the design might adversely affect or interfere with the human aspects of the process. These issues ultimately led us to this present thesis topic, the identification of guidelines for digital Cardwalls informed by field studies of software development teams.

\subsubsection{Trello}

Trello [64] was created by Fog Creek Software during the course of our own project. Perhaps more than any other digital tool for story management, Trello captures the simplicity of the traditional physical Cardwall. The simple design allows flexibility in how it can be used. Trello can align with many different workflows, from simple to-do lists as seen in Figure 2.9, to Agile development as seen in Figure 2.10, and also to other personal, business or management applications. 


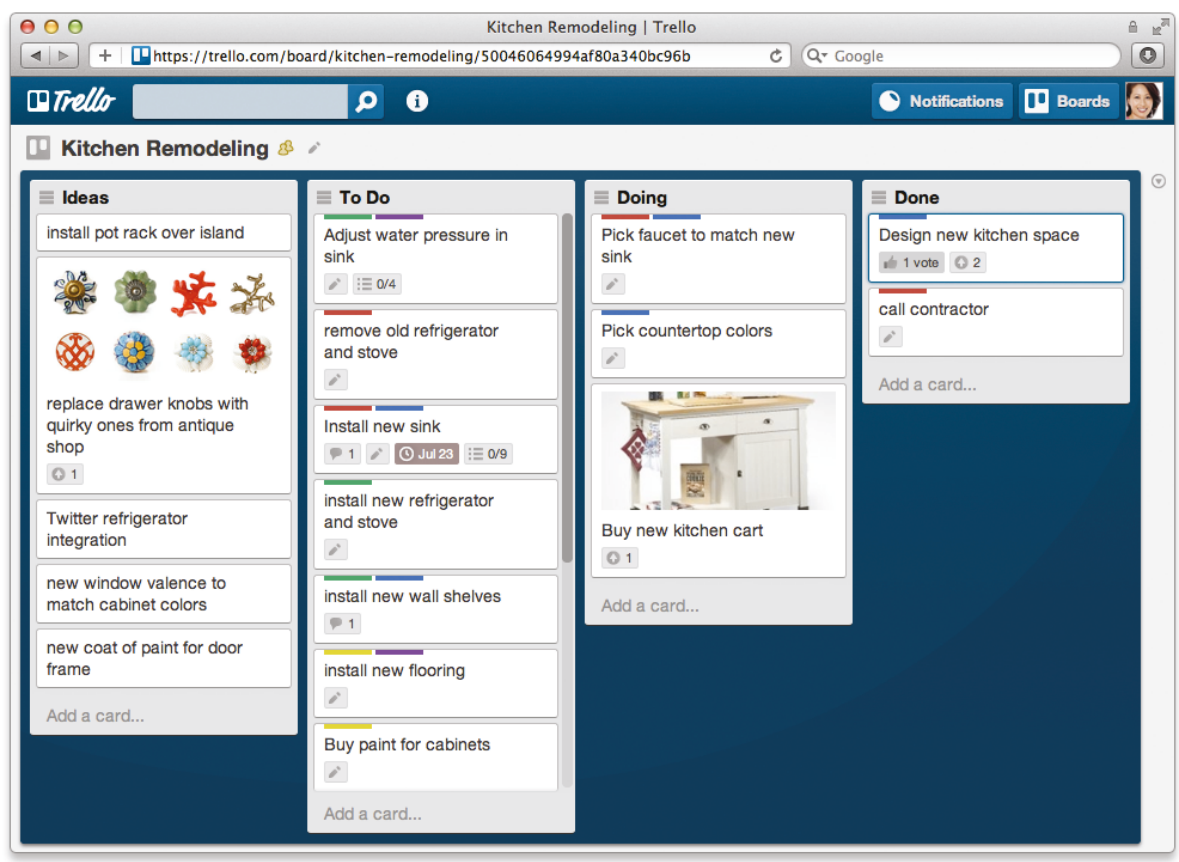

Figure 2.9: An example Trello board for managing household events (http://www.trello.com/tour)

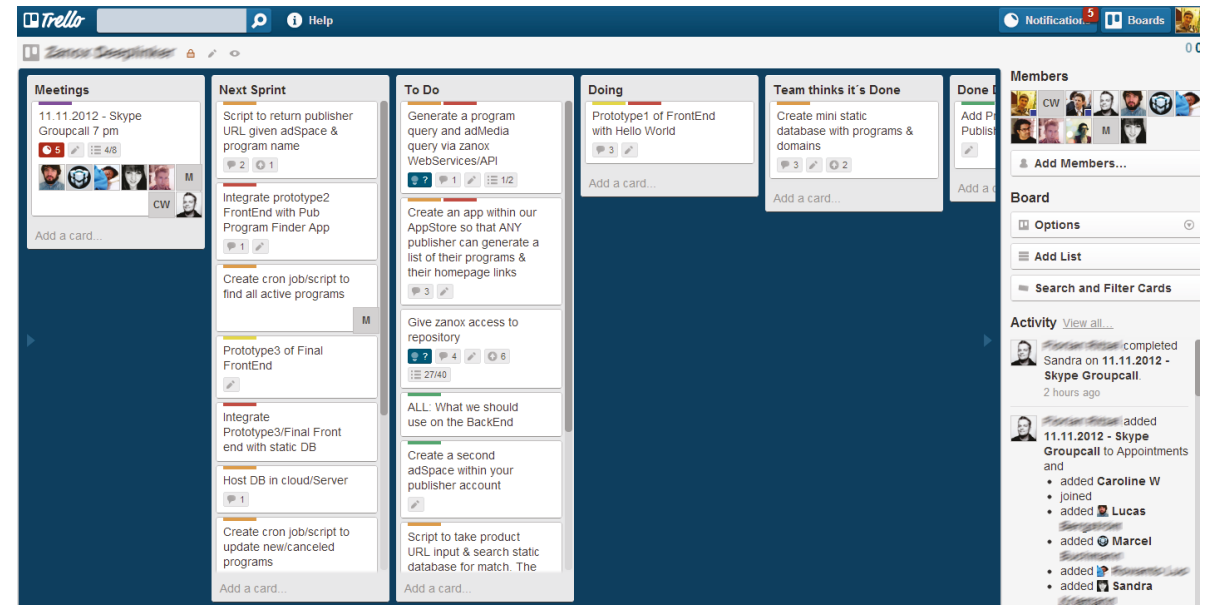

Figure 2.10: A Trello Cardwall for a software development project (http://www.agileinberlin.com/) 
Each new board starts with three empty lists titled: Todo, Doing and Done, but you can add as many lists as you want, each with its own title. Similar to the Storycard, in Trello you add new content to a list by clicking the "add a card" button at the bottom of any list. The cards have two views, a minimal view used while viewing the board at large and a detailed view, where you can see and edit all the extra content that is hidden on the back of the card. Each list can grow arbitrarily and the cards allow the user to easily add rich content, such as images and URLs or even embedded videos. With Trello, everything is saved automatically so there is no need to remember to save or update. On the Trello homepage, they explain that the simplicity of their applications allows users to use it in a flexible manner that can reflect the way they think or the processes they follow.

Trello has been designed very well from a user interaction perspective, but it does have limitations. For example, it is not possible to view the details of a Storycard while still viewing the Cardwall. Similarly, one can only see the details of one particular story which makes it difficult when planning and the discussion involves the details of more then one story. Finally, Trello is not designed to support simultaneous, co-located multi-user interaction which may have an impact on its support for collaboration.

\subsubsection{Story Repositories}

JIRA [5] is an issue tracker developed by Atlassian [3]. JIRA allows software development teams to track and assign issues as well as help to track the activity of teams and their members. Issue Trackers, also known as bug trackers, ticket support systems, or management workflow systems, allow teams to enter and track the progress of whatever the particular system is designed to track. They provide useful search features and reporting capabilities, including graphs to help visualize the progress, and reports for management. Such systems are not really designed to support Agile planning with User Stories, but are widely used for this purpose in practice.

JIRA is probably the most popular issue tracker and has been adopted and re-purposed by Agile software development teams to manage their User Stories [13]. The major impetus for this development was that Agile teams were looking for a solution to the distributed team dilemma where team members working from different locations had no access to the team Cardwall. The use of JIRA by Agile teams for this purpose became so popular that a 


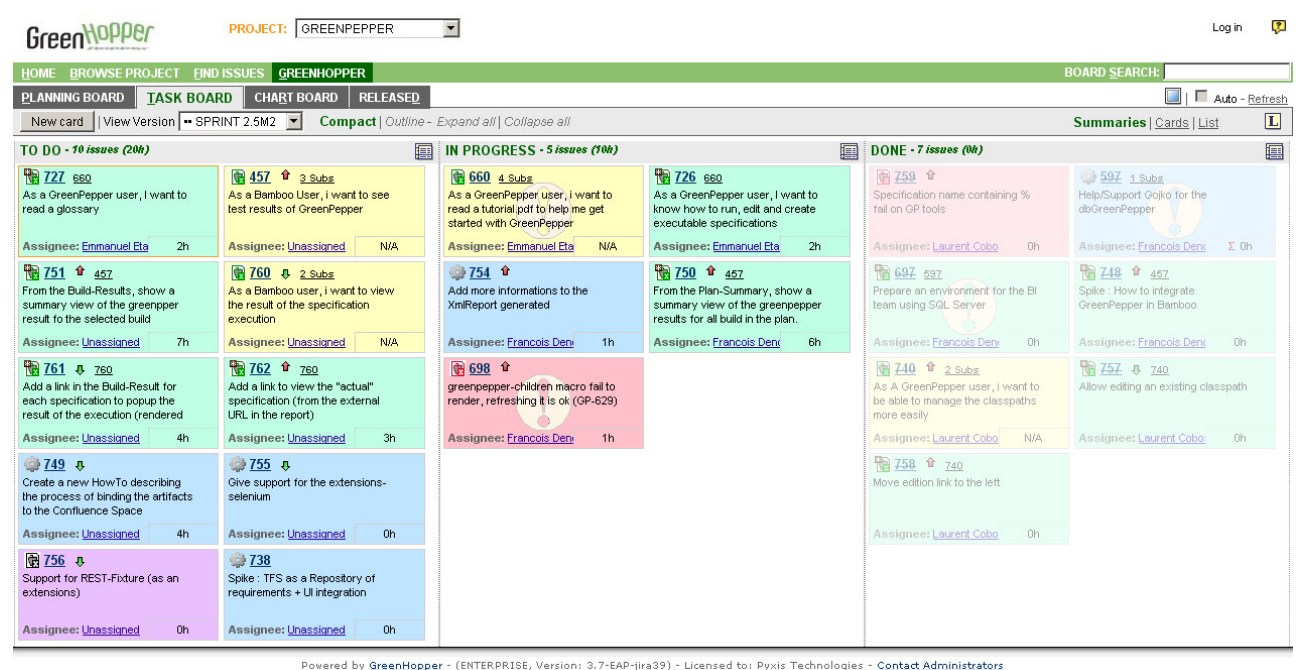

Figure 2.11: A Cardwall using the Greenhopper plugin for JIRA (http://blog.softartisans.com/2012/01/25/better-living-through-user-scripts/)

plugin called Greenhopper was developed to add a Cardwall view on top of JIRA as seen in Figure 4.1. Development for this plugin is on-going and the name has been changed to JIRA AGILE. This new version offers a sleeker view as seen in Figure 2.12.

Several of the teams involved in our field study, which will be described in Chapter 4 used JIRA in combination with the Greenhopper plugin. 


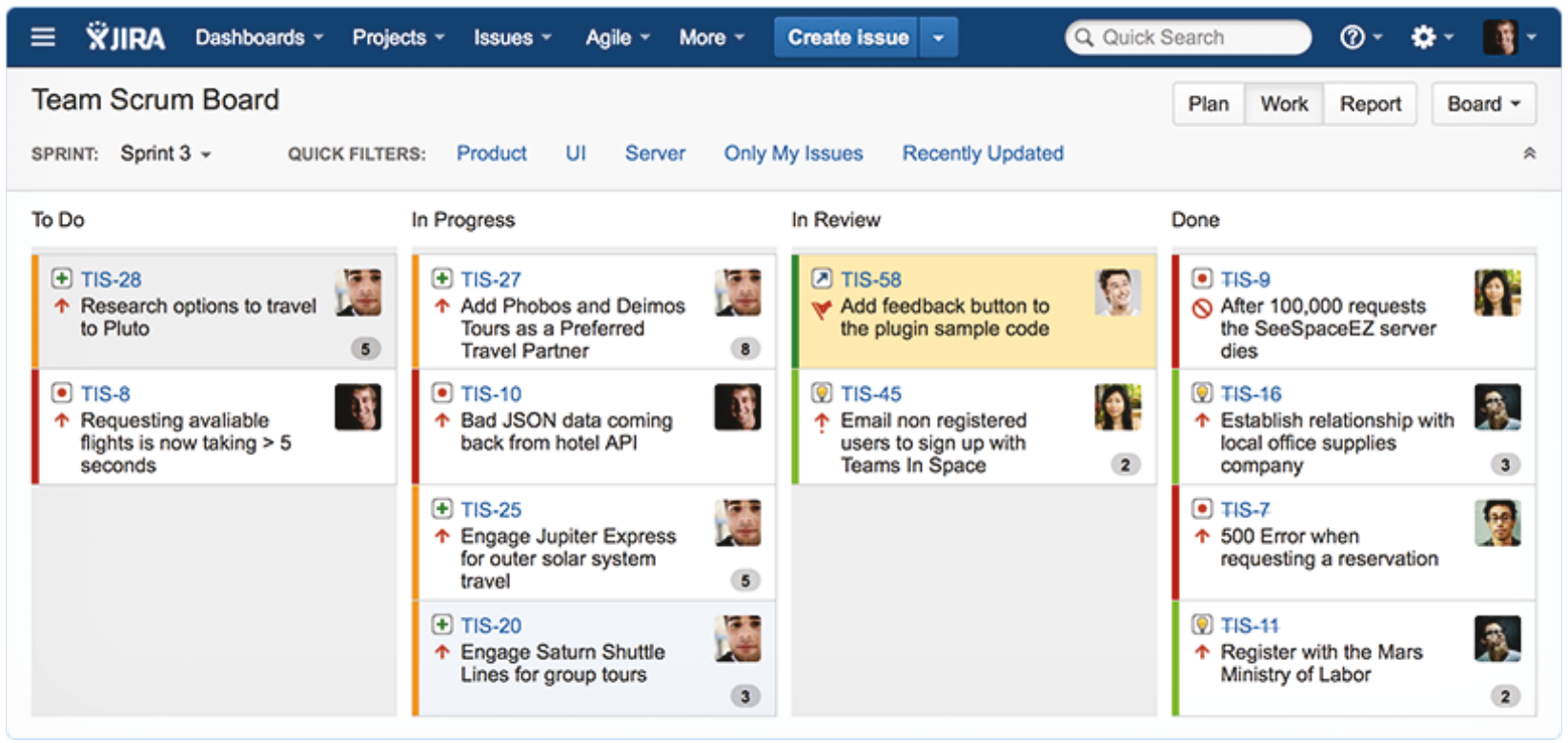

Figure 2.12: A typical Cardwall used by the teams in our study using the JIRA/Grasshopper type of Cardwall. Each card is associated with an issue in JIRA. Such Cardwalls were projected on walls in team meetings. Source: [4] 


\section{Chapter 3}

\section{The Pilot Study}

Since the conception of this project, we wished to explore how a digital Cardwall might leverage most, if not all, of the advantages of the traditional physical Cardwall, while also gaining digital advantages. Based on our review of the literature, we had developed an informed understanding of the subtle features of physical Cardwalls that contribute to their effectiveness, and in turn their popularity, as a highly productive lo-fi, low-cost method for progress tracking and project planning. Among those many advantages, we believe that the interactions fundamental to Cardwall use are in themselves instrumental to the success of physical Cardwalls. The simplicity of these interactions have impressive power; this includes moving stories, annotating them and finally removing them at the end of an iteration. Added benefits of physical Cardwalls are greater organizational transparency, their support for increased communication within the teams, and especially with the customers. The increased project awareness is among the most important reasons that physical Cardwalls have been adopted by so many real-world Agile teams.

Even with this understanding from the literature, we still felt it was critical to observe real Agile teams during planning meetings and status report meetings where the collaboration was focused around the team's physical Cardwall. For the preliminary study, our goals were modest but important for the design of the field study to come. We wanted to get a sense of how real Agile teams use Cardwalls, including how closely this aligned with the literature. We hoped to identify opportunities to further inform the design of a digital Cardwall, but above all, we wanted to inform the design of our larger study, and in particular the design of the semi-structured interview questions.

The pilot study consisted of informally observing an Agile software development team working for the Government of Canada. Later, as part of our larger study, we then followed up by conducting a formal interview of the project manager using the approved protocol of our field study (see Chapter 4). In the interview, the project manager elaborated on the 
details of their process and the artefacts they used. Their process involved daily standup meetings and iteration planning meetings. From the interview we identified many interesting behaviours which helped strengthen our understanding of the use of physical Cardwalls in practice.

This team used three Cardwalls that were simultaneously maintained on adjacent walls. One Cardwall, a backlog called "The Scope Bank" by the team, was organized in rows such that each row contained stories roughly grouped by sprints. The top-most row contained the stories for the next sprint. The next row contained the stories for the sprint after that and so on. The collection of stories in the rows was carefully determined for rows 1-3, but very roughly determined after that. The team's backlog was really a one-column wall.

The second Cardwall was a little more traditional, in that it had the typical multi-column layout. Each column represented some stage in the team's work-flow; each row represented one story, but it had been decomposed into tasks. Colour coding was used to represent a role. Therefore, a glance at a card would identify a task, the role responsible for executing the task, the state of the task and the story it supported.

The third Cardwall was more of a task board and intended for non-software related tasks. For example, they would have tasks to remind the customer that he needed to find out some information or talk with some end user, etc.

The use of Cardwalls by the pilot study team was not traditional, in that they had three distinct Cardwalls on display, one for the prioritization of the backlog, one for the current iteration, and one for non-developer related tasks. They also had a dedicated meeting room where their walls were on constant display, but not close to the team. The ability to support diverse use of the Cardwall was an important point which was also backed by the literature.

The interview revealed some problems with identifying related stories. For example, the prioritization of the backlog involved shuffling stories in and out of each row. Sometimes decisions were made to remove a story from the top row of the backlog; when this happened someone from the team would point out that if that particular story was moved then so should another one because they were dependant and there was no point in working on one story if the other one was not going to be touched. Similarly, when stories were being moved into the top row, sometimes other related stories would also have to be moved. In either case, finding related stories was difficult and involved looking through stacks of stories to 
find the ones of interest. We thought at the time that a well-designed digital Cardwall would certainly help with this task.

In summary, the interview helped our awareness of some important aspects of Cardwall use relevant to our research goal. Firstly, we gained awareness of the importance of the backlog and how a well-designed Cardwall should be able to support flexible content and should facilitate prioritization. Secondly, we found that a digital Cardwall would need to be able to easily identify and have some method of visualizing different possible types of relationships that stories could share. Thirdly, we found that Cardwalls needed to support customization and a flexible design for teams with multi-disciplinary team members.

We developed our field study, and especially the interview questions, to specifically explore these issues. We wanted to frame our future investigations with our preliminary findings, but we also wanted to keep an open mind with respect to our future participants' experiences with Cardwalls, which we expected would reveal other issues. 


\section{Chapter 4}

\section{Field Study of Digital Cardwall Users}

\subsection{Motivation}

As we described in Chapter 2, the Story Cardwall is a central artefact used by many Agile practitioners to support project planning and maintenance. Considering the simplicity of the Cardwall, it may be surprising, and not entirely obvious, how it supports key roles so effectively. However, deeper investigations have identified how this tool supports the planning and maintenance of software projects, how it enables focus and transparency, how it fosters awareness through its role as an 'Information Radiator' [15], and how it tends to inspire ad hoc conversations which help with motivation and innovation.

Through our literature review and our pilot study, we gained an informed understanding of the benefits of physical Cardwalls as well as a clear understanding of why the Agile community in general recommends the use of physical Cardwalls as a best practice tool of choice. However, we now wanted to understand how to design a digital Cardwall which could capture the known benefits of physical Cardwalls and provide additional functionality only possible with a software solution. We imagined many possible features easily implementable with a digital solution including support for distributed teams, backups, traceability and automated reports. However, we wanted our design to be informed by the actual needs of real-world Agile teams. We therefore conducted a field study using observations and interviews to explore the possibilities.

The list of available tools to help manage Agile projects using users stories is growing, however, none are specifically designed for multi-touch technology nor are they designed specifically for large high resolution displays. We envisioned a Cardwall that would leverage both those technologies to help support the known advantages of the physical Cardwall, while allowing the introduction of new functionality only possible with a computerized system. In particular, the multi-touch-capable design should consider the benefits of physical touch, and 
the use of gestures, and the way that multi-touch affords opportunities to support collaboration in terms of real-time, simultaneous, co-located use. Designing specifically for large high resolution displays should allow us to replicate physical Cardwalls and the way they are being used, but still potentially innovate through the use of new kinds visualizations and interactions.

To achieve these goals, we designed a study to investigate how modern Agile teams use digital Cardwalls to understand more of the reasons leading to the adoption of digital story Cardwalls and the frustations experienced by teams using digital Cardwalls.

\subsection{Research Questions}

The goal of our study was to increase our understanding of how Agile teams use digital Cardwalls and stories to support their software development activities. In particular, we were interested in answering the following research questions.

Q1: In what ways do current implementations of digital Cardwalls and stories meet the needs of distributed Agile teams?

Q2: What usability issues exists with the use of digital Cardwalls and stories?

Q3: Can we identify features that are difficult, inadequate or missing from existing digital Cardwalls and story implementations?

\subsection{Method}

\subsubsection{Participants}

The target participants of this study were established professional Agile teams that used digital story Cardwalls. We wanted to understand how these teams collaborated around and used their Cardwalls. To achieve this awareness we decided to conduct a field study where we would be able to simply observe Agile teams in their place of work collaborating around their walls during planning or evaluation meetings. 
Because observations can capture behaviours, but not motivation, to gain even deeper understanding, we also wanted to interview a handful of key team members who had participated in the observations. This would give us the opportunity to further understand the observed interactions, helping us to develop insight, as well as to collect some distinct personal opinions about the use of story Cardwalls. Our goal here was to interview one to three team members per project. Ideally we aimed to interview the project manager, one novice and one senior team member, but this was mediated by opportunities and constraints.

We decided to conduct a week-long field study that would consist primarily of observations, but that would also include a day or two of interviews. The participants (Agile Teams) of this study were selected opportunistically, mostly through connections made while attending Agile 2011 conference in Salt Lake City, Utah. During the conference, we made many potential contacts that we followed up with via email. We sent the potential teams all the information on our project, including the informed consent forms for both the interviews and the observations. We clearly explained that participation was completely voluntary and anyone (team or individual) could decide to opt-out at any point, during or after study and if this happened they would not be reprimanded in any way. We explained that we were not interested in the actual software they were developing, but rather, the Agile process they were using and in particular their use of Stories and Cardwalls.

\subsubsection{Procedure}

The study was conducted in two parts, the first part was an observational study of professional Agile software teams as they collaborated around Cardwalls during planning or evaluation meetings. We used ethnographic methods [10,41,53]. We were interested in observing daily stand-ups, Iteration Planning Meetings (IPMs) and retrospectives. We were also curious about the role of the digital Cardwall in short daily stand-up meetings. The observations were to be conducted in the place of work of the different teams so as to try and maintain the highest level of ecological validity possible.

The second part of the study consisted of individual interviews. Similar to the observations, the interviews would be conducted in the place of work of the participants where it would be less likely that participants would experience undue anxiety or stress. Our goal was to secure an available office at each organization we visited in order to conduct the interviews 
in private. The informal, semi-structured nature of the interviews would allow us to explore the personal experiences with Cardwalls and stories of Cardwall use from each participant. For these, we also used semi-structured interview methods [26,41,51,63]. We also aimed to understand our participants' overall impressions about the tools they used, including points of frustration and their insights on how things could be better.

\section{Materials and Equipment}

We decided to conduct our field study in the regular workplaces of our participants, therefore all the materials and equipment needed were already an integral part of their activities. We expected that the teams would have some subset of the following standard equipment at team meetings: laptops or personal devices, such as smart phones or tablets, some form of shared display (monitor, tv, laptop or projector) and a high-speed Internet connection. The latter we expected would be useful for facilitating the participation of remote users, for conducting quick searches, for accessing team resources, or for demonstrating live software. We also expected the Internet connection would be useful for finding and comparing features which may have been implemented in other existing software. Most importantly, we expected, at the very least, that each meeting would have one large shared display for their Cardwall, as this was a requirement of participating in the study.

The only additional materials needed to conduct the study were a note pad, pen and an audio recording device for data collection. The materials for the observation session (we thought we might audio record some) and interview were the same.

We also used a typical informed consent form and debriefing form (See Appendix B).

\subsubsection{Analysis}

The observational and interview data were to be collected, summarized and typed up (in the case of the observational notes) or transcribed (in the case of audio recordings). The typed notes and transcribed audio recordings would be imported into ATLAS.ti, a tool designed to support qualitative research in the data-analysis phase.

We decided to use thematic analysis $[25,29]$ to analyse our data; this would allow us to identify common themes across the interviews and observations through the creation of codes. To code, we would highlight interesting bits of information and tag them with an appropriate 
label, such as "Cardwall Enhancements", or "Story Grouping". The process of coding is manual and laborious, requiring a significant amount of attention to the identification of significant details, as well as the development of concrete codes and abstract themes, which would refer to collections of concrete codes that address a similar issue.

Once manually coded, ATLAS.ti has a number of features which can help researchers identify 'saturated' themes. In particular, the number of times or frequency of a code, i.e., the number of quotes identified and tagged with a particular code, is a good indication of the richness of the data with regard to that code. We planned to combine this information with the co-existence of other high-frequency codes to identify themes. While this type of coding is often used to develop new theories, via a process called Grounded Theory, our goal in this analysis was to inform the design of a multi-touch digital Cardwall.

\subsubsection{Ethics Approval}

We described the study and applied for approval from the Carleton University Research Ethics Board. The full protocol is shown in Appendix B, and was approved with clearance number 12-0416.

\subsection{Results}

In this section we present results from our analysis of the observational and interview data and speculate in general terms about the design consequences of these results. This section is organized by themes. These themes emerged as the result of the coding process and are topics commonly raised during the semi-structured interviews or incidents commonly observed across projects.

\subsubsection{Overview}

The field work spanned two cities, one in Canada and the other in the United States of America.

Including our pilot study (which we include here because it was instrumental in confirming our understandings of physical Cardwall use), we observed at four different organizations/employers. Table 4.1 provides demographic information about the four organizations 
Table 4.1: Organizations Visited

\begin{tabular}{|l|l|l|l|}
\hline Organization & Client Organization & Location & $\begin{array}{l}\# \text { of Projects } \\
\text { Observed }\end{array}$ \\
\hline Octagon & CareCo, & Chicago & see next row \\
& NetClinic, & & 1 \\
& St.Mary's HS, & & 1 \\
& MichealetBot, & & 1 \\
& 8 to 18 & & 1 \\
\hline CareCo & Internal & Chicago & 2 \\
\hline Pilot Study & Internal & Ottawa & 1 \\
\hline Rand & Internal & Ottawa & 1 \\
\hline Total Organizations: 4 & & & Total Projects: 8 \\
\hline
\end{tabular}

we visited. (Note that here and throughout the thesis we use pseudonyms for all proper nouns.) At two organizations, we studied multiple teams.

All of the teams participating in our study used Agile-like processes for development work. In particular, they all used Scrum to manage their projects, although the language they used (e.g. to describe a sprint) varied slightly from team to team. Two of the organizations were closely connected and had very similar Agile practices (Octagon and CareCo); these included: pair-programming, daily standup meetings, sprint planning meetings and retrospectives. These two organizations also practised Software Craftsmanship [19], which, although Agile-like in many respects, distinguishes itself through its strong emphasis on mentoring for the purposes of accelerated skills development and general understanding, and a constant exposure to best practices through case studies, workshops, invited speakers and the like. The particular practitioners of software craftsmanship we observed reserved Fridays as a day for personal development, by allowing their team members to work on open source projects of their choice. This allocated time for personal development was aimed to keep teams motivated and allowed their developers to gain exposure to other real-world projects.

Our participants were organized into eight teams, each one responsible to a client organization (See Table 4.3). The majority of client organizations were based in Chicago and were introduced to us through Octagon, an Agile development firm we met while participating in the Agile Alliance 2011 conference in Salt Lake City, Utah [1]. Octagon is a Chicago-based development firm whose main income stream is through the acquisition of contracts for development work. For these contracts they would either establish a predominantly in-house team 
Table 4.2: Project Table

\begin{tabular}{|l|l|l|l|}
\hline Project & Client Organization & $\begin{array}{l}\text { Agile } \\
\text { Process }\end{array}$ & Type of Cardwall \\
\hline Babysitter WebApp & CareCo & Scrum, XP & JIRA \\
\hline Nanny WebApp & CareCo & Scrum, XP & JIRA \\
\hline Online Insurance Claims & NetClinic & Scrum & StoryBoard \\
\hline Athletics Camp & St. Mary's HS & Scrum & StoryBoard \\
\hline Online Survey Creation & MichealetBot & Scrum & StoryBoard \\
\hline Event Scheduling WebApp & 8 to 18 & Scrum & StoryBoard \\
\hline Auditing Software & Pilot Study & Scrum, XP & Physical \\
\hline WebApp & Rand & Scrum, XP & JIRA \\
\hline Total Projects: 8 & & & \\
\hline
\end{tabular}

Table 4.3: Team Table

\begin{tabular}{|l|l|l|c|}
\hline Client Organization & Team's Composition & Team's Project & Team Size \\
\hline CareCo & 5 client, 2 Octagon & Babysitter WebApp & 7 \\
\hline CareCo & 5 client, 2 Octagon & Nanny WebApp & 7 \\
\hline NetClinic & 3 client, 7 Octagon & Online Insurance Claims & 10 \\
\hline St.Mary's HS & 2 client, 6 Octagon & Athletics Camp & 8 \\
\hline MichealetBot & 2 client, 6 Octagon & Online Survey Creation & 8 \\
\hline 8 to 18 & 2 client, 5 Octagon & Event Scheduling WebApp & 7 \\
\hline Pilot Study & 7 client & Auditing Software & 7 \\
\hline Rand & WebApp & 10 \\
\hline Total Participants & & & $\mathbf{6 4}$ \\
\hline
\end{tabular}

to do $100 \%$ of the development work, or they would provide developers and sometimes project leads to work directly with the client's own development teams. Either way, the teams were always composed of employees from both Octagon and the client organization. For example, CareCo (an organization we visited for two days to observe and conduct interviews) had contracted Octagon to help two distinct in-house development teams with their work on two different projects. For both these teams, out of seven total members, two members per team were employed by Octagon. The remaining Octagon clients, listed in Table 4.3, consisted of teams that were mostly composed of Octagon employees and for which most of the work was conducted at the Octagon head office, and Table 4.3 shows the client organizations, team compositions and team sizes.

In all, we observed teams at work on eight different projects. These projects used one 


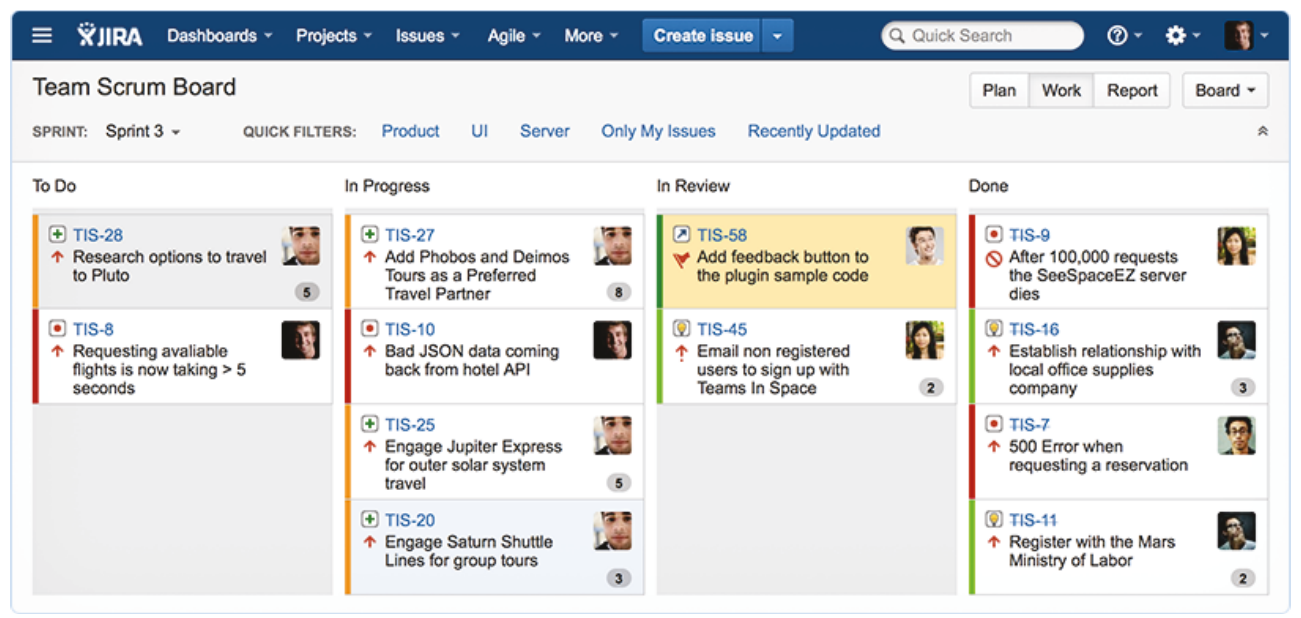

Figure 4.1: A typical JIRA/Greenhopper type of Cardwall. Each card is associated with an issue in JIRA. Such Cardwalls were projected on walls in team meetings. Source: [4]

of three following tools as their Cardwall: JIRA/Greenhopper digital Cardwalls, an in-house digital Cardwall called StoryBoard, and a physical Cardwall created with post-it notes and tape. See Table 4.2 for details on the projects and Figures 4.1,4.2, \& 4.3 for Cardwalls like the ones that we observed. Table 4.2 also shows the Agile process and type of Cardwall employed for each client organization.

We observed 13 meetings where stories and Cardwalls were used, including daily standup meetings, iteration planning meetings (IPM), and sprint retrospective meetings. Table 4.5 provides key information about each team we observed as well as the types of meeting we observed. The observations gave us the opportunity to see the real work-flow around Cardwalls, specific behaviours with respect to Cardwalls, and to see problems that might arise due to the type of Cardwall in use.

There were sixty-four participants in our study. From these participants, we opportunistically selected seven for in-depth, private interviews. The selection was based on their role, availability, and willingness to participate. Due to time constraints demographic data was only collected for the interviewed participants and can be seen in Table 4.4, which identifies the participant's, role, experience, organization and the project on which they worked. Note that the organization column (from Table 4.4), refers to the actual employer of the participant. The participants varied substantially with respect to their role on the team. Two were novice developers and five were experienced (including three project managers). Table 4.6 


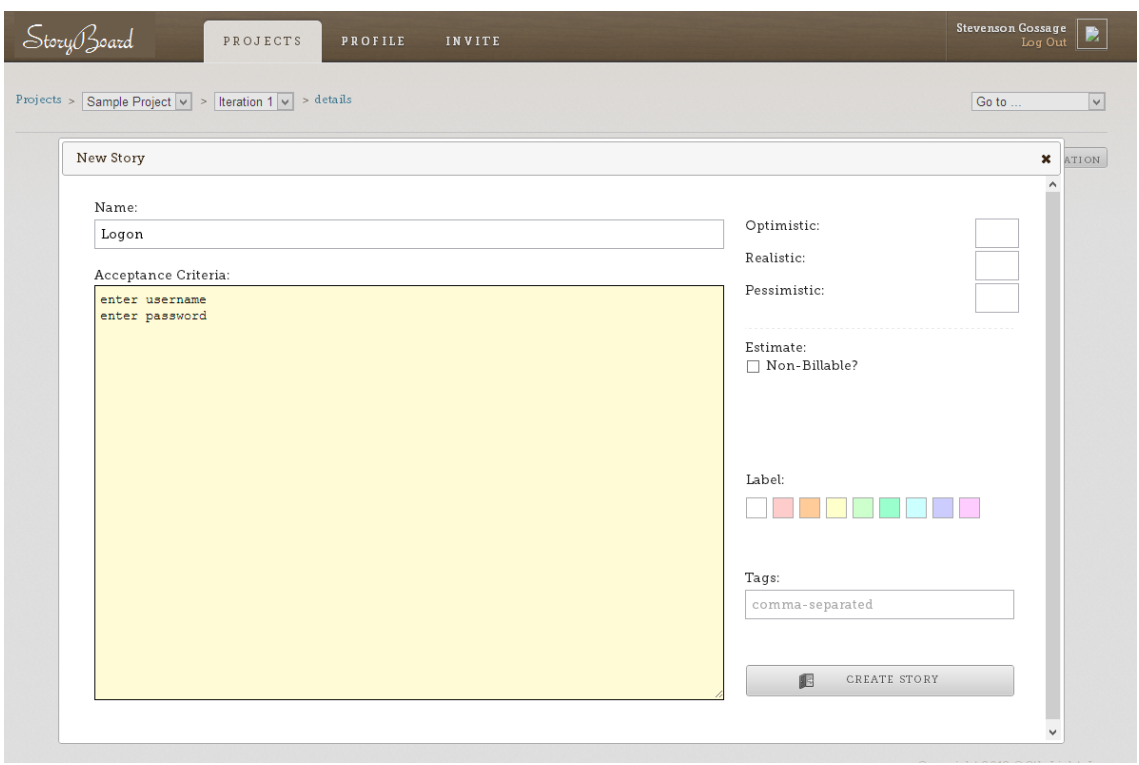

Figure 4.2: A story from the StoryBoard Digital Cardwall. This tool was developed inhouse.

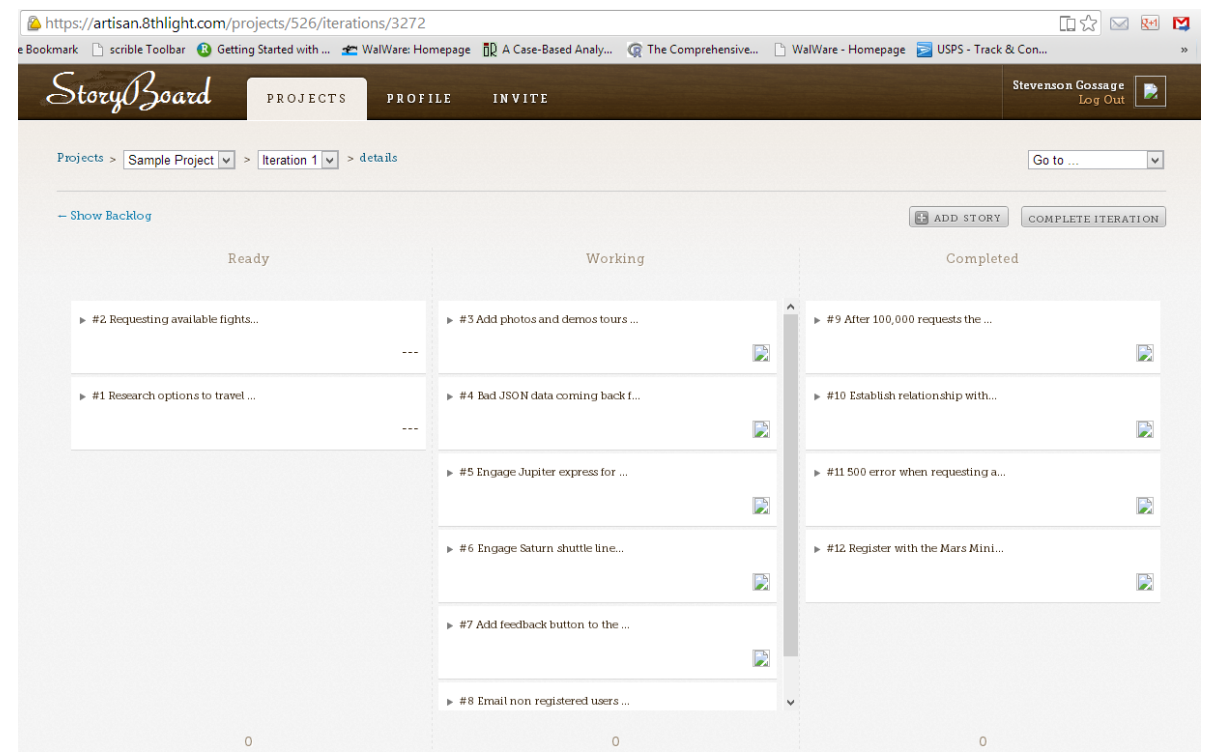

Figure 4.3: An example of the StoryBoard Digital Cardwall. There are 3 fixed columns: Ready, Working and Completed. This simple layout was typical of the StoryBoard Cardwalls we observed. 
Table 4.4: Demographic Information For Interviewees

\begin{tabular}{|l|l|c|l|l|}
\hline Name & Role & Experience Level & Employer & Project \\
\hline Mitchel & $\begin{array}{l}\text { Software } \\
\text { craftsman }\end{array}$ & Inexperienced & Octagon & $\begin{array}{l}\text { Babysitter WebApp, } \\
\text { other web sites }\end{array}$ \\
\hline John & Developer & Experienced & CareCo & $\begin{array}{l}\text { Nanny WebApp, } \\
\text { other web sites and }\end{array}$ \\
\hline Wayne & $\begin{array}{l}\text { Software } \\
\text { craftsman }\end{array}$ & Experienced & Octagon & $\begin{array}{l}\text { Babysitter Nanny WebApps, } \\
\text { other web sites and }\end{array}$ \\
\hline Michael & $\begin{array}{l}\text { Project Man- } \\
\text { ager }\end{array}$ & Experienced & CareCo & $\begin{array}{l}\text { Nabysitter Wanny WebApps, } \\
\text { others, various } \\
\text { platforms }\end{array}$ \\
\hline Dennis & $\begin{array}{l}\text { Project Man- } \\
\text { ager/CEO }\end{array}$ & Experienced & Rand & various WebApps \\
\hline Keith & $\begin{array}{l}\text { Software } \\
\text { craftsman }\end{array}$ & Inexperienced & Octagon & Sitter WebApp \\
\hline Donnie & $\begin{array}{l}\text { Project Man- } \\
\text { ager }\end{array}$ & Experienced & Octagon & $\begin{array}{l}\text { Multiple projects, } \\
\text { various platforms }\end{array}$ \\
\hline Jack & $\begin{array}{l}\text { Project Man- } \\
\text { ager }\end{array}$ & Experienced & Pilot Study & Auditing Software \\
\hline Total: 8 & & & & \\
\hline
\end{tabular}


Table 4.5: Observation Table

\begin{tabular}{|l|l|l|l|}
\hline Events & Client & Location & Project \\
\hline Daily Standup & CareCo & Chicago & Babysitter WebApp \\
\hline Daily Standup & CareCo & Chicago & Nanny WebApp \\
\hline Daily Standup & Rand & Ottawa & WebApp \\
\hline Iteration Planning Meeting (IPM) & CareCo & Chicago & Babysitter WebApp \\
\hline Iteration Planning Meeting (IPM) & CareCo & Chicago & Nanny WebApp \\
\hline Iteration Planning Meeting (IPM) & NetClinic & Chicago & Online Insurance Claims \\
\hline Iteration Planning Meeting (IPM) & Pilot Study & Ottawa & Auditing Software \\
\hline Iteration Planning Meeting (IPM) & St.Mary's HS & Chicago & Athletics Camp \\
\hline Iteration Planning Meeting (IPM) & MichealetBot & Chicago & Online Survey Creation \\
\hline Iteration Planning Meeting (IPM) & 8 to 18 & Chicago & Event Scheduling WebApp \\
\hline Retrospective & CareCo & Chicago & Babysitter WebApp \\
\hline Retrospective & CareCo & Chicago & Nanny WebApp \\
\hline Retrospective & NetClinic & Chicago & Online Insurance Claims \\
\hline Total Events: 13 & & & \\
\hline
\end{tabular}

provides details about each interviewed participant. Experienced interviewees had more than five years experience in software design and development, a figure which correlated closely with their experience with Cardwalls and stories. Inexperienced participants had five years or less experience on software teams. All participants had experience on previous projects because most of the current projects in were newly established.

Table 4.6: Interview Table

\begin{tabular}{|l|l|l|l|}
\hline Participant Name & $\begin{array}{l}\text { Duration of } \\
\text { Interview }\end{array}$ & Employer & Project \\
\hline Mitchel & $35 \mathrm{~min}$ & Octagon & Babysitter Webapp \\
\hline John & $43 \mathrm{~min}$ & CareCo & Nanny WebApp \\
\hline Wayne & $34 \mathrm{~min}$ & Octagon & Sitter Web Site, Other Web Sites \\
\hline Michael & $55 \mathrm{~min}$ & CareCo & Babysitter \& Nanny Web Apps \\
\hline Dennis & $45 \mathrm{~min}$ & Rand & Web app \\
\hline Keith & $37 \mathrm{~min}$ & Octagon & Babysitter WebApp \\
\hline Donnie & $43 \mathrm{~min}$ & Octagon & Babysitter, Nanny, NetClinic \\
\hline Jack & $30 \mathrm{~min}$ & Pilot Study & Windows App \\
\hline Total Interviews: 8 & & & \\
\hline
\end{tabular}

We asked interviewees about their experiences with Cardwalls, both current and past. We asked questions about behaviours we had seen while observing Cardwall use in the pilot 
study and asked 'why' questions about their particular preferences and tendencies when using stories and Cardwalls. In this way we were better able to understand the behaviours we had observed and also the historical and organizational context of Cardwall usage.

\subsubsection{Themes Identified}

In all we developed 57 different codes, however, many of these were not saturated [18] and we eliminated these from our analysis, leaving 15 codes which we grouped thematically (See Table 4.7), which lists the most used codes per identified theme. The following sub-sections describe the six themes that we identified based on the thematic analysis, our data and the application of our codes. Throughout, we used quintessential quotations from our participants that effectively expressed the message we received from them as a whole.

Table 4.7: Codes used in the analysis of our data grouped by Themes

\begin{tabular}{|l|l|}
\hline $\begin{array}{l}\text { Corresponding } \\
\text { Themes }\end{array}$ & Codes \\
\hline $\begin{array}{l}\text { Flexibility Advantages } \\
\text { with Digital Cardwalls }\end{array}$ & $\begin{array}{l}\text { big picture, distributed, resources, simple, Cardwall_digital, Card- } \\
\text { wall_enhancements, Cardwall_location, Cardwall_meetings, Card- } \\
\text { wall_backlogs, Cardwall_planning, Cardwall_process, physical vs } \\
\text { digital, Negatives, Positives }\end{array}$ \\
\hline $\begin{array}{l}\text { Challenges in Present- } \\
\text { ing Detailed Informa- } \\
\text { tion }\end{array}$ & Negatives, Positives, physical vs digital \\
\hline $\begin{array}{l}\text { Exploring and Filter- } \\
\text { ing Information }\end{array}$ & $\begin{array}{l}\text { story_acceptance criteria, story_alignment, story_annotating, } \\
\text { story_components, story_grouping, story_traceability }\end{array}$ \\
\hline $\begin{array}{l}\text { Managing Backlogs } \\
\text { story_activities, story_creation, } \\
\text { story_expectations, story_prioritization, software delivery, sprints }\end{array}$ \\
\hline $\begin{array}{l}\text { Multi-disciplinary use } \\
\text { of Stories }\end{array}$ & $\begin{array}{l}\text { roles_Cardwall user, roles_customer, roles_designer roles_developer, } \\
\text { roles_end user, roles_product owner, roles_project manager, } \\
\text { roles_QA, roles_stakeholder, roles_various }\end{array}$ \\
\hline $\begin{array}{l}\text { Keeping the Cardwall } \\
\text { Updated }\end{array}$ & $\begin{array}{l}\text { foster_awareness, foster_comunication, Cardwall_visibility, collabo- } \\
\text { ration, story_views, story_testing, story_states, story_ownership }\end{array}$ \\
\hline
\end{tabular}

\section{Perceived Advantages and Disadvantages with Digital Cardwalls}

This first theme is an important one, but at the same time it is quite general and many ideas that come out in this theme also emerge in some of the themes that follow. As with other 
themes, the content of this theme is based on our notes of team meetings and our interview transcripts. With respect to this theme, in the transcripts, our participants expressed both negative and positive views about both digital and physical Cardwalls, and we capture these sentiments in this theme. In general the advantages and disadvantages covered in this theme all touch on the idea of flexibility and how the digital Cardwall provides more ways to do things, but also how this can over complicate the usability of the tool. The remainder of this section organises the perceived advantages and disadvantages with the following categories: wall and user location, complexity, relationship visualisation and traversal.

Wall and User Locations: The teams we observed were mostly using projectors to display their Cardwalls. This was especially true of the Cardwalls used for external customer projects (most of the projects), but not so true for Cardwalls that were created for internal projects.

Projected Cardwalls have the advantage that they are portable and can be used in any available meeting space where there is a projector. When used in this way, we observed that one member of the team (usually the project lead) was responsible for updating the Cardwall as the team worked.

Keith, a novice developer had used a physical Cardwall on a previous project. Keith liked the presence of the Cardwall, but also admitted to forgetting to update it at times. Keith said:

"Yeah, so with the physical one [Cardwall], its just a lot more visible, I guess. Even though the virtual one, the (online one ) is easily accessible. But the one in the workplace is just easier (you can sit ) back, and ... have a full view of what's in progress".

Another disadvantage of physical Cardwalls is the poor fit between physical Cardwalls and the distributed work of teams, which is an increasingly common arrangement for software projects. One or more team members could be located off-site, working from home or travelling. In fact, there was at least one distributed (remote) participant at each of the iteration planning meetings as well as in some of the daily stand-up meetings. Remote users communicated via Skype [45], GotoMeeting [14] and teams 
even used telephones when the other two technologies were not co-operating (something that we witnessed in two meetings).

A disadvantage of physical Cardwalls is the limited physical space teams have for display purposes. In the environments we observed, there were many small projects, and they shared common meeting rooms for their team meetings/team events. In fact, the requirement for wall space outgrew the physical resource.

Because customers attended these meetings it was not appropriate to display Cardwalls from other projects; the projector system allowed the team to have only one Cardwall visible at a time, and made efficient use of wall space.

A slight point of frustration that was expressed was all the extra steps that were necessary for a digital Cardwall to work. Among others, Michael expressed this point, but also indicated that because of the distributed nature of their teams a digital Cardwall was necessary:

“... in general I do like the card wall we have[JIRA/Greenhopper]. I've used just physical card walls and they're more effective in terms of being able to rapidly capture thoughts and easily move things around. ... That said, we work remotely with Developers and Resources so a physical card wall just isn't possible in our situation."

Complexity: Wayne speculated about problems with maintaining either physical or digital Cardwalls, especially when projects were large. For digital Cardwalls, he saw the problem of page refreshing as an annoyance, but with physical Cardwalls he saw clutter being a challenge because of space limitations.

"If there were maybe a larger team with a longer sprint, there would be so many cards that it might help to physically rearrange them [to reduce clutter]. But we're not in that situation."

Michael, talked about how he would love to see even higher level overviews of the project and not just concentrate on the current iteration. He explained:

"I think one of the biggest shortcomings currently with our card wall solution is, within an iteration it works pretty well, but if we want to zoom out 
and see a story map of the entire backlog, there's not a view available. So, I think, in terms of understanding the larger context and being able to visualize that, I think that would be a great benefit for everyone involved in the project. But it's not something that we can do with our current tool. [JIRA/Greenhopper]"

Interestingly, the added benefits afforded by the digital environment can be viewed as either a blessing or a deterrent. Deterrents that can have a negative impact on the team's willingness to use a feature. For example, Michael, one of our project managers, loved how you could link JIRA with other tools and described this feature as important and easy to use. Michael said,

"One of the things that JIRA does really well is when developers commit code, the first part of the comment is the key of the issue in JIRA, and as they commit, there's an integration between GitHub, which is our source code management and JIRA. So, Commits are linked to JIRA issues. And then within JIRA you see all of the activity related to that particular issue. So I think that simple linking mechanism has incentivized people to use it more. Because now the team knows we have a complete traceability from: here were the requirements; all of the information about the requirements, the status, history is captured; and then a link to the actual changes in source code."

At the same time Wayne, one of the developers working with Michael recounted his frustrations relating to the same issue of code change notifications, he said:

"I get emails when stuff changes ... it's like, [if] my updates happening 10 times, I get 10 emails", which "is completely meaningless to me because there's too much information."

Wayne recounted, "My inbox is sacred. I don't want to use email to notify me that stuff is happening. I feel it's the wrong medium". He did not want to be flooded with meaningless code change emails. We concluded from this and other similar stories that it is extremely important to consider how changes and relationships, dependencies etc. 
can be visualized so that we accomplish the goal of increasing team and individual awareness without creating unnecessary distractions and annoyances.

Relationship Visualisation and Traversal: In our data, participants mostly described how digital stories enhanced their work and had the potential to enable it further. Wayne used JIRA to track stories and said stories acted as a helpful guide for his development work. "All I need is a few sentences telling me what to do, and a name for those few sentences." He sees stories as "buckets of criteria [requirements]", but would like to easily see relationships between stories.

In the physical Cardwall we considered, in our pilot study, the visualization of relationships between stories was supported through the use of simple methods like their use of colors, annotations, and rows. The rows (commonly referred to as swimlanes) enabled the team to visualize the flow of stories that had been been grouped together based on their relationship to a particular role. In general, swimlanes are used to visualize the flow of related stories, where the stories from each row can be worked on independently from the stories in the other rows. The grouping can be based on virtually any relationship useful to the team and examples may include the role of the end user, the role of the team, the software component or feature that the stories are part of. In the electronic Cardwalls we observed, we saw support for some of these same simple methods, but we did not see teams attempt to visualize any more sophisticated types of story relationships. We later learned from the interviews that the ability to see these relations was either difficult to do, or not supported by the tool. When discussing how a digital Cardwall could be used to visualize different types of relationships, Michael said:

"I think the key to success there is the linking mechanism. In JIRA they do have the concept of a link. But you have to click more actions and then link, and then you have to pick the type of link, and then you have to search, and then you have to try to find it, and then you click it, and then you click another thing, and it's too much. It's way to hard to do, so nobody does it."

Dennis and his team used JIRA and would have liked to see relationships between stories made more evident. When they worked, Dennis explained that his team kept 
track of dependencies between cards "in their heads" because not only could it not be done via the Cardwall, but in general "we don't have good dependency tracking tools." Dennis also believed it was useful to look at the evolution or history of stories because he wanted to see a hierarchical understanding of stories and how they evolve from large epics to smaller implementable stories. "I think it's very, very important to be able to look at the evolution." Dennis' interests then were in linking dependent stories and, like Wayne, linking stories in a hierarchy from epic stories on down to the type of stories seen on a typical Cardwall, i.e., stories that can be completed within one iteration. However, Dennis was frustrated with performance issues in general, "the performance of the tools is just terrible! I mean humans are very fast relative to most software," and he believed that any tool he would want to use would have to perform better than the tools they were currently using.

Another related issue that was also mentioned by various team members was the inability to navigate quickly between connections. John, one of the more experienced developers, recognized the benefits of being able to decompose and reconstruct epics into smaller, more manageable stories, but also thought it was important to be able to leverage these relationships as a way to quickly jump from one story to another,

"Ideally, if you can split an epic [into multiple stories] and keep that tied back to the epic ... that would be really nice to be able to just go-to-it [the story or epic] depending on which direction you wanted to go".

\section{Challenges in Presenting Detailed Information}

Large higher resolution displays are another way to display more content; but the physical size of Cardwall elements may be a more important driving force towards favouring larger, rather than higher resolution displays. Extremely high resolution may be more important for personal displays and portable devices as high resolution text is easier to read and causes less eye strain. Small touch-enabled portable devices like smartphones may have extremely high resolution, but the user experience is of utmost importance and the designers of these systems balance the added higher resolution with the knowledge that the interactive components and widgets of the applications must still be a size that is sufficiently large so that the fat finger problem is avoided. This same issue also applies to text, the added resolution makes a 
font-size appear much smaller than on a device with less resolution, so application designers must also find a sweet spot that works for the majority of the target audience. Similarly, collaborative applications specifically designed for either high-resolution displays or tiled displays must also consider how text and other artefacts will be viewed and at what distance.

For the interactive touch enabled Cardwall, the addition of a touch interface may further complicate this because, like the fat finger problem found on smartphones, the interactive application components on a high-resolution display must not be rendered so small that they become difficult to use. The trade-off between the size and resolution of displayed objects is a key for Cardwalls, and this point was expressed by our participants who were concerned that individual objects (such as Storycards) need to be big enough to be understood, but not so big that a big picture can't also be discerned. Dennis described the problem by saying,

"So I mean I could easily be biased to use a card wall if the cards were fairly small if I could see them, if I had a mirror, if I could slide over the card and [see a balloon view] so I could see the full description so I can press a lot of cards into the screen [itself] you know...I don't think I'm really that biased against Cardwalls it's just that the current ones the cards are either too big or too small."

Dennis raised concerns about inflexibility in the size of physical or digital cards, especially when there were many cards. "If they're ... too big [you can have problems] if you want to actually manipulate a whole lot of cards. And if they're too small, you put them all up there and you can't read them.". Denis complained that with existing Cardwalls "the cards are either too big or too small." Dennis said that we had to find the right balance "between the manipulation of many cards or reading a single card. Need to do both." When asked about about his experience with the JIRA Cardwall, another participant, Keith, replied, "It's OK. It's a little cluttered."

\section{Exploring and Filtering Information}

Wayne, one of the experienced team members, described his desire to visualize the relationships between stories. He envisioned "a robust framework for indicating that this story came out of this other story" and something to indicate "here's what its original form was" and something that would tell him "here's the spawn of that story." He'd like to see stories linked 
in various meaningful ways, especially closely-related stories, but also previous versions of a story or even a story's "history".

The difference between novice and experienced Agile team members is most evident when participants were asked about their perception about how stories and Cardwalls facilitated awareness. For example, one experienced Agile practitioner, Michael, describes how Cardwalls help him keep track of the status and progress of multiple projects of which he was involved, "To get an idea of where things are from a status perspective. So, I have this team, but I also have five other projects that are going on." Appendix A contains a table with quotes from both experienced and novice team members.

In contrast, less experienced Agile practitioners like Keith would focus more on the physical and visual details of the Cardwall, which he felt helped increase the team's overall awareness. For example, he described the use of colors to indicate the likeliness a story would be completed. In essence, the more experienced team members were more concerned with the big picture than the less experienced team member.

Opinions about the importance of grouping, filtering, tagging and linking stories, as well as how and to what extent their current electronic Cardwall supported these features was another point where the experience of the team members made an evident difference. The more experienced participants envisioned how added functionality could help increase awareness about important, but currently invisible, aspects of their projects. For example, Dennis explains that he would like to see the software move beyond simple grouping and allow him to retrieve vital information about a certain grouping of stories, he says he would like to be able to ask the Cardwall questions like: "how long will this [group of stories] take,..., how many defects are [associated with this group], who's working on [this group], [and] are we behind?" the less experienced team members focused more on the rudimentary ways they currently were able to group stories like Keith mentioned how they would use colour to identify bugs and tests: "tests were yellow, and bugs were (pink or red)."

Dennis suggests that one useful method of grouping stories would be by software component, so that the team could select different components and see the associated stories, the progress of those stories etc.,

"We'd like to be able to put the stories into different places so we can see who's impacted and so on, and again, most systems don't allow you to, for instance, 
design and tag the things by what component [the stories are affecting]."

More experienced team members, like Michael, recognized how the Cardwall and the use of stories were actually themselves catalysts for many of those same conversations,

"I think that people need to see that overall context and need to be able to go up to the Cardwall and have conversations around it, because if you're just in the weeds you lose the [big] picture."

By comparison, less experienced team members also found the Cardwall useful for keeping them up to speed on the progress of other team members, "We usually met around the Cardwall. You'd take a look at what's up and what we have started or finished", but they were not necessarily convinced that the Cardwall was the best medium to help increase their individual awareness about the progress of their team's efforts. In fact, Keith says that he feels he can achieve similar levels of awareness solely through the ongoing conversations between team members, "Usually everyone was talking and knows [as a result, everyone] what everyone's doing..."

Michael also described features that he would like to see available in his digital Cardwall (he is using a JIRA Cardwall) that would help him remain aware of business goals. He said,

"It might make sense to do things differently from a technical perspective. And that process of reworking the stories is painful. So I don't know exactly the solution, but it would be great to be able to rapidly create new things that represent the technical and tie them together with the business requirements."

As a manager, he recognized the importance of being able to easily drill down from higherlevel, more abstract ideas to lower-level, more detailed oriented issues. For example he felt that the ability to visualize features on a Cardwall would be ideal for meetings with high-level executives but, at the same time, the ability to drill down and see the epics that could be associated with each feature, and in turn see how each epic was broken down into stories, would help increase the overall awareness of the projects' real progress and help provide a more realistic understanding of the work involved in the implementation of a feature. In contrast, inexperienced developers did not discuss business goals.

The opinions of our participants about the value of Cardwalls and user stories differed based on the years of experience with software design and development. In general, we 
observed that experienced team members envisioned more sophisticated, yet simple features that would help increase the visibility of connections between stories, their history, how they relate to higher level requirements, components or even business objectives and goals, all of which could be used to justify the existence and even the prioritization of stories. Less experienced team members were generally less likely to recognize the importance of such features because they tended to be less focused on the big picture and more concerned with the day to day progress of the current sprint.

Dennis complains about using Greenhopper with JIRA when working on large projects with huge backlogs. "Rand does not [generally] use Greenhopper. There's too many stories and too many cards. You have to have a useful UI rendering. [In JIRA we] can't allow ranges for dates or hierarchies of stories. It's also slow. Some people do use it though." Dennis continues to explain that the underlying databases used by systems such as JIRA do not have a useful data model for the purposes of searching and filtering data relevant to stories and iterations. He says, "Well, the problem is they're fast but, again, you're representing hierarchical information you need a different data model than they do - most of these most of these things are really designed about, you know, basically storing a story as a key." In this way, issue trackers like JIRA, which have been re-purposed by Agile teams to manage their stories are not able to efficiently search and filter since often stories are stored as blobdata using a single key. The use of this model means that queries are extremely inefficient since key terms of interest to Agile teams and key components from story artefacts are not indexed or directly searchable from the underlying database.

\section{Managing Backlogs}

Opinions of the significance and management of backlogs differed based on the size of the project the team was working on. The backlog is a significant element for large projects; the ability to see the backlog's stories, and to sort and prioritize the stories becomes increasingly important as the size of the project increased. However, larger projects tended to have larger backlogs, which themselves can be displayed on a Cardwall, as happened with several of the project teams that used StoryBoard and JIRA/Greenhopper, both of which allowed developers to drag stories from their backlog onto a Cardwall. This evolution of the use of traditional Cardwalls is an ideal example of how a digital implementation can enhance the 
traditional physical Cardwall.

The interviews helped us understand how the teams were truly self organizing, and helped us understand the process that teams followed when choosing stories to work on. In particular, we found team members were very interested in the prioritization of the backlog and how the stories were selected for each new iteration. We also learned how stories were chosen by individual team members during an iteration, as well as how teams would manage the exceptional case when a developer was left without an available story to work on.

When we asked about how stories are assigned, the general answer was that they were not assigned, that each team member would simply pick a story from the ready column and start working on it. Wayne explained as follows:

"So, if there's something in progress, and a person wants to pair, I'll do that. Like, I'll just ask, see if he wants to pair. And if he didn't, so I pulled the thing out of the Ready column. Now, as soon as I finished that, I'm gonna help my other teammate on a story that's in progress. So, ... I guess, its not complicated."

Wayne continued to explain that noone forces a story on anyone and that he liked this because he felt it meant you would work on parts that you might not otherwise get to see. He said, "Yeah, it's really good to have responsibility and to get exposure to parts of the app which I might not touch otherwise, if I was specialized in one component." Wayne explained that the stories in the current sprint all shared the similar priorities and that it was before the start of an iteration, during its planning (the IPM), when prioritization was critical. When asked about the priority of the stories ready for development Wayne replied:

"No, I think that once we commit, we should try and get them all done. And if it's a week, one week sprint, like, it's not terribly high overhead. Like just do it all, and if one thing came out, it was as important as the other ones. For a one-month sprint maybe there's a disparity in the priority. But for one-week, if we're actually doing the highest priority stories, there's not much difference between the top and the bottom. I think the top prioritization is in IPM."

Mitchel explained how the prioritization would actually occur in the backlog: "So there's the concept of the In Progress list, and those are not sorted by priority. Those are just a bucket of things to be done. The backlog is the only thing that's 
really sort of high priority. So the backlog, whatever is first is most important, that goes into the bucket first."

So if a developer was done and no one wanted to pair, then the stories would come from the backlog. Keith explained how his team decide to use colours during IPMs to identify extra stories, not included in the iteration but that could serve as a mini-backlog from which developers could pull from if needed. Keith said:

"We'd usually get the iteration done, and if we didn't then we'd - or if we were done super-early we would grab from the backlog anyway, ... Yeah, we would ask [the product owner], it's like, we had, we ended up having like a red, yellow or a green, yellow, red sort-of-section. So, green: what we are pretty sure that we're gonna get done this week. Yellow: we think we still can, but it's possible it won't get done. And then, red. So we had enough [stories] so that we should have extras."

In our study we saw how teams sometimes used different backlogs for infrastructure type work that needed to be done, but that did not come from the stories created by the customer. Keith explained how the team started this process after a retrospective, which identified a need for lower-level components. He explained:

"We ended up creating, like, a Dev backlog for stories that developers wanted to do. Like clean up something, or, you know, look into some new technology or something else. So we put that on a separate wall nearby that just had things that we wanted to do, and whether you're working on it. You would just remove it if it was done."

Sometimes, teams wanted to use the backlog to store information that would be useful for planning, estimating and reporting purposes. Dennis explained:

"Bugs are also put into the backlog. Vacations are also put into it. This lets managers understand what is going on if the velocity is reduced or if they're on vacation and training. Everything is thrown into the backlog. This makes for visibility." 
Dennis continues to explain how this is important for overall team awareness and planning.

"If I'm gonna be completely lean and agile, I want to use a single mechanism for doing that. So we like to throw everything in there [the Backlog], um, or encourage people to, not everybody does this, right, 'cause then I can see, right, I know that this - that there are really only three people there 'cause everyone else is off on vacation, otherwise we'd get bogus reporting systems and metric systems that trigger and say 'look the velocity [...]' velocity of team mates gone down, and so on, and then you go in and find out it's because, ye know, people are working on three projects, or they're on vacation or training or something. So we like to have complete backlog."

Dennis' team was also generally less concerned with the priority of stories, but as deadlines approach and and a large large release was close, he admits that, "When we're down to the end of the final time boxes for the - the release, we're - we're gonna be a lot - a lot more concerned about prioritizing what's on the backlog."

\section{Multi-disciplinary use of Stories}

The interviews made it abundantly clear that stories are vital to the designers and project managers and that they enjoyed and valued working with them. Digital stories in particular were a key artefact for all the participants.

In our study, the process surrounding the use of Cardwalls was basically the same across teams. Teams tended to use basic Cardwalls comprised of three columns: to do, in-progress and done. However, some teams extended this simple arrangement with the addition of extra columns thereby increasing the number of states and providing those teams with a better representation of their work-flow and their more sophisticated processes.

In most meetings, we also observed that individuals in roles other than the developer role also interacted with Cardwalls through stories. Although initially designed for the developer community, Cardwalls are becoming a multi-disciplinary team tool. For example, we observed

designers and testers organizing their work around stories. However, at the same time we also noticed that non-developers had different requirements for Cardwalls, i.e., the useful information radiated from the Cardwall was different for non-developers. 
We noticed that the effectiveness of the information radiator aspect of a wall depended on the role of the team member using the wall. For example, for developers and designers, the constant display of the current sprint is very useful for increasing the awareness of the status of the project, and it can even serve a motivational function. However, for the team's testers, their interest in the project is probably limited to the list of stories that require testing. For example, if the wall has a testing column, the tester may only need to have access to that part of the developers' Cardwall as a single column to develop an awareness of the size of their task. This suggests that filtering of Cardwalls would be a useful operation. However, for testers, it would also be important to understand the role(s) each story has in terms of the overall system under development. Further, for proper regression testing, and some other more complete whole system testing it would be to their benefit to be able to visualize what components are touched by each story. This could help identify the interfaces that need testing.

One new possible feature that was discussed was different ways of linking external but related artefacts. For example Michael describes how he would like to link their automated acceptance test with their Cardwall. They use an automated testing suite called Cucumber, where each cucumber represents an acceptance test, Michael explains the process:

"So initially Product is writing the Cucumbers. There's a team collaboration to do refinements. Once work is committed then developers are actually linking the Cucumbers into the code and making them pass. QA is also collaborating with developers to make sure that it's done properly, and owns the overall organization of the entire test suite. So there is some manipulation that happens after Product originally writes the Cucumbers. But that's all done between DEV and QA, and Product's out of the loop at that point because it's within GitHub. So we haven't found a good way to maintain that collaboration. And for me that would be through JIRA, since everybody's using that tool and understands that tool and is used to collaborating there."

\section{Keeping the Cardwall Updated}

It is sometimes easy to forget to update a digital Cardwall, especially if it is more of an issue tracker and not an actual physical Cardwall. A physical Cardwall is updated at least once 
a day during the daily stand-up. Also, physical Cardwalls act as information radiators and are generally in a shared space where the team can easily see them. It can be satisfying to touch the wall, grab a story and move it to the "Done" column. The walk to the wall can be enjoyable as can the visibility of the action. Whereas with digital Cardwalls this same action is not as visible and sometimes even forgotten as describe by Keith,

"Sometimes I forget to move the cards, and that does cause problems. I've had a time where I started working on something where someone else had already started as well, but they forgot to move the card. So, that was a problem. It ended up being not a ton of time down the drain, but it was a little bit."

Keith also explained, that sometimes forgetting to update the digital Cardwall could go completely unnoticed, "That's caused problems before. Other times, I mean, you just get lucky, or people just know what you're working on."

Michael describes the updating process in general.

"There's multiple ways that we're updating the Cardwall. One is during the IPMs where we're scheduling items into a sprint. And that's done through a laptop. Usually one person is driving, and making sure that all the requirements are in place, updating the estimates from development. And then once we kind of triangulate on how much work the team can commit to in an iteration then we'll start the sprint. So that's done in the IPM as a team with one person driving. After we start the sprint, then developers are self-selecting their work and they're updating the Cardwall themselves as it progresses [through] the various statuses."

Michael continues to describe how the flow is interrupted by the need to constantly do extra steps, "There are some inefficiencies when you go to a digital solution. You have to click on things, you have to type things, you have to save things, it's just slower all around." John also found the electronic process slower explained his issues:

"So, I mean, collaborating should be multiple, right? All directions, not just one. So I mean, if I want to hand off to the customer, [or] go ahead and write the story, you can't do that. You have to update it and then refresh. Right? That's the only way to sort of collaborate. It slows things down. And it's just not ideal. 
I mean, if you were right there on a Cardwall, a physical one I mean, if you go back to the physical idea of where this comes from, you're just sitting there and you're just doing it right there. You're both there and you can do it. And that's natural. That's why we used to use [physical] Cardwalls."

\subsubsection{Analysis Bias}

Because the analysis was conducted by an individual we need to explain how this could introduce bias into our analysis. The computer science background of the individual as well as the years of experience working as a developer for different companies influences the way the researcher sees and interprets the data including the codes used and even the quotes selected to highlight interesting detail. Regardless of the bias, we strongly believe that the general findings and themes identified through the analysis of the data collected in this study would be similar if analysed by other researchers, since the presence of the identified themes was so strong within our data.

\subsection{Research Questions Answered}

\subsubsection{Distributed Agile Teams}

Our first research question was, "Do current implementations of digital Cardwalls and stories meet the needs of distributed Agile teams?"

As projects increase in size, the need for supporting distributed team members collaborating around a Cardwall is increasing and the need is becoming unavoidable. Current digital Cardwall solutions support distributed teams in that their systems generally allow multiple simultaneous access. Remote users can login and see the same view as their co-located team members. In general this strategy requires that local Cardwall updates be saved to the server or cloud and sometimes remote clients may even require a refresh to force synchronization between clients so that changes can be seen. In addition, there is no digital Cardwall solution that supports all aspects of a collaborative distributed Agile team meeting using the Cardwall as the central artefact. To support this type of team meeting, the system might benefit from integrated support for both voice and video, although the meetings we observed always used some other software like Skype for voice and/or video communication. 
While distributed teams members can now login and see the same view, it is not clear how effective current solutions are at drawing the user's attention such that changes are easily seen even by remote participants. Creating this type of team awareness, where the attention of all participants can be drawn to notice changes is critically important and remains an open opportunity for further investigation and research.

\subsubsection{Usability of Digital Cardwalls}

Our second research question was "What usability issues exists with the use of digital Cardwalls and stories?" It was clear that current digital solutions were not necessarily easy to use, personalize or adapt to a teams specific needs. For example, filtering was difficult, so much so, that we did not observe a single occurrence, the issue only came out during the one-on-one interviews. Other issues, like the lack of control over the granularity of email alerts, where users would complain that when they are bombarded with updates the value is lost and eventually the 'update' emails are simply ignored. It was apparent that updating and remembering to save changes was also an issue. Usability is one of the most important aspects that directly correlates with product adoption. This is why these usability issues need to be understood and addressed in the design of our Cardwall.

\subsubsection{New or Improved Features}

Our third research question was "Can we identify features that are difficult, inadequate or missing from existing digital Cardwalls and story implementations?

There were several opportunities identified for new functionality whose implementation could have real implications on awareness, not just at the project or iteration level, but also at higher organizational levels. Among these features, the ability to easily visualize hierarchical relationships and dependencies between stories appears to be critical in terms of fostering awareness at different levels of the organization. Also important and related to the first two points is the ability to decompose epics into stories and re-construct those epics from the stories. The ability to easily link or create associations between stories with their history (including the reasons for the stories and why a story may have changed or been split). Linking business goals and software components was also a requested feature that was currently unsupported. 


\section{Chapter 5}

\section{Implications for Design}

This chapter considers the findings of our field study in terms of implications for design, which we express as a series of guidelines for the design of a digital Cardwall. We structure the remainder of this chapter and frame the guidelines based on the insights we developed in the analysis work of the previous chapter, theme by theme.

\subsection{Digital Cardwalls}

In this section, we start by addressing some important issues that should be considered by the Agile teams wishing to convert to a digital Cardwall, but also to teams already using one. We offer our recommendations for how best to leverage the advantages of the digital Cardwall so that the team gains all the benefits of this medium while retaining the advantages of the physical one. Our recommendations are based in part on the work of Sharp et al. [60] and their findings about the social context and how it serves as the glue which binds the lightweight Cardwall process to its successful use in practice.

One advantage of the digital Cardwall is the flexibility in terms of where it can be displayed. This advantage is important because it allows teams to conduct meetings in shared locations like meeting rooms, which provide a degree of privacy and confidentiality (a need we frequently observed). This means that teams working on sensitive projects can reap the benefits of a Cardwall, while their customers can rest assured that their sensitive data is not compromised. This also has the added benefit of reducing the requirement for wall space and allows meetings to be conducted in any office or boardroom using existing equipment.

A down-side to this practice is that teams lose the benefits incurred from the always on and constant display of the physical Cardwall. There is evidence in the literature for the value of large physical Cardwalls and their role as "Information Radiators" and their ability to draw attention, increase awareness and support spontaneous conversations which in turn

enhance the overall understanding and communication of the team and their customer. The 
fact that the traditional physical Cardwall is large, and situated close to the team enables rich and effective communication. Teams must not overlook these benefits and should also consider using a dedicated display, located in a shared space close to the team. In this way, teams can benefit both from the flexibility of this 'display anywhere' solution, but also from having the Cardwall available in a central dedicated location.

Overall, we have found that the issue is not whether a physical or digital Cardwall is better, it's about how to design the advantages of a physical Cardwall into the digital Cardwall. The following guidelines have been identified to help with this challenge.

Big Picture: Large displays facilitate team planning work and are ideal for capturing a big picture view of the project. In our field study, all of the iteration planning meetings and retrospectives used projected images of the team's Cardwall which was large enough for it to be easily seen at a distance by all participants. We also observed that during IPMs, each story being considered as a candidate for the next iteration was viewed and discussed in detail. Fortunately, large displays have enough screen real-estate such that a group of co-located collaborators can easily view the details of one or more stories. From our observations, the short stand-up meetings were generally not held in meeting rooms and they did not generally have access to a large view of their Cardwall. For these meetings, the big picture Cardwall was used more as a point of reference similar to a cue card, and was ideal for keeping the meeting focused and on point. Guideline: Always support a large format overview of the entire project to facilitate team and stakeholder discussions.

Concurrent Multi-User: Although the teams we observed were using digital story Cardwalls, these tools were limited in their support for concurrent multi-users. Their Cardwalls allowed distributed users to access and view the Cardwall from their remote location however, there was no support for real-time concurrent use in terms of editing and viewing the details of more then one story simultaneously. This sometimes caused inconveniences, where the details of distinct stories of interest had to be remembered instead of simultaneously viewed. Guideline: Allow simultaneous multi-user access.

Flexibility: The appearance and layout of the digital Cardwall will change based on the display format. An ideal design would also consider how user interactions change 
depending on any combination of the following factors:

- the number of concurrent users

- the physical location of the users (co-located or distributed)

- the medium used to display the Cardwall (smart personal device, desktop monitor, large shared display)

We observed Cardwalls displayed on large shared surfaces for the meetings which were held in meeting rooms, but also on laptops or smaller desktop displays. The meetings were composed of mostly co-located participants, but often there was as at least one remote user. For each scenario the answers to the questions above change, this creates a challenge for detailed upfront design. Furthermore, although the teams we observed were not using smaller personal devices like smart phones or tablets, these devices may be an important part of the interaction story and a mobile version should be considered and designed for. In general, with a web-based Cardwall, there could be any number of distinct form factors used for display, ranging from smaller personal devices to larger shared displays. Guideline: Support diverse screen sizes with appropriate design.

Direct Interaction: In our field study, we consistently saw one member of the team (usually the project manager) interacting with the Cardwall on behalf of the rest of the team. This kept the work tightly coordinated, but could also slow it down. The ability to interact directly with the Cardwall through physical touch would be ideal, especially to help the team maintain awareness about the changes. The added ability to have multiple team members interact simultaneously would also help for collaboration. Guideline: Support direct multi-touch where possible.

\subsection{Challenges in Presenting Detailed Information}

One advantage of digital Cardwalls is directly related to the size and resolution of the potential display area. As seen in our study, the use of projectors provide adequate support for simply enlarging what normally would be displayed on the a user's laptop or regular desktop monitor. This solution does not leverage specific design principles for use with large, high-resolution displays, nor does it derive any benefit from the increased screen real-estate 
because the same number of pixels are being displayed. The cost of large screen displays has dropped significantly, especially for displays that support a resolution of 1080P or less. Newer displays with $4 \mathrm{~K}$ and $8 \mathrm{~K}$ display resolutions are now available but, for the most part, the cost of theses displays is still quite high. That said, if your large display has the same resolution as your single user laptop or personal desktop display, then there may be no real design guidelines to follow to help leverage the increased size. For example, 1080P is a popular resolution which can be found as the native resolution on many larger TV displays including ones that are over sixty inches on the diagonal. Similarly, many modern desktop displays also support 1080P as do the more expensive laptops. For our design, we consider $1080 \mathrm{P}$ as the default resolution, but we recognize that this resolution can be found natively on displays ranging from smaller hand-help devices to massive displays used in stadiums. For displays with truly high resolutions like the $4 \mathrm{~K}$ and $8 \mathrm{~K}$ displays or tiled displays, guidelines can be gathered from the literature for creating visualizations and usable software on these types of high-resolution surfaces [21,24,32-34,71].

Arguably the most important role of the Cardwall is its ability to communicate the status of a project to team members and stakeholders. This means that the big picture requirement described above is critical, but we are also often concerned with the details. With the digital Cardwall it is possible to hide details, but allow users to display them when needed. However, the ability to view the details when planning and discussing stories and iterations has been identified as a general requirement based on our study. Also, because we saw how important a role the Cardwall played during collaborative planning meetings, and because of the way the traditional, physical Cardwall is used, we have spent a considerable amount of effort on the design guidelines specific to the interaction of co-located users collaborating around the Cardwall. We believe this is the most important scenario and we believe that it will also be the most common in practice. With this in mind, the following guidelines are also based on Shneiderman's Visual lnformation-Seeking Mantra: overview first, zoom and filter, then details on demand [61].

Overview First: We previously argued for the need to support diverse screen sizes, but for a Cardwall the ability for users to quickly assimilate the information from a reasonable distance is critical. There is a delicate balance between the amount of information that can be displayed and the amount of information that should be displayed. Regardless of 
the nature of the currently displayed information, whether we are currently visualizing the big picture or the details of a story, the displayed information must be easily discerned from a distance. It is impossible to know exactly what is too big or too small and at what distance users will be standing from the Cardwall while collaborating. None the less, since our goal is to try and leverage the benefits of the physical Cardwall, we will make the assumption that whatever is on display should be understandable from a distance of approximately five to ten feet from the Cardwall. Guideline: Always begin with and default to a general overview display.

Zoom and Pan: As identified in the previous section a Digital Cardwall can be viewed on virtually any display; it can be difficult to know the actual size of the display and the resolution. To aid the user, it should be possible to re-size the current view of the Cardwall to accommodate the display being used.

When zoomed in sufficiently such that parts of the current view are no longer visible, panning allows the user to easily access the parts of the view that are hidden. Guideline: Support zoom and pan to allow users to leverage diverse displays.

Details on Demand: As described above, the details of any particular artefact are not always important and therefore should not always be present. However, a Digital Cardwall needs a mechanism which allows users to show and hide details whenever they are available. Guideline: Allow optional display of details that would otherwise clutter the current view.

Awareness via Visual Cues: It is important that users can easily recognize when there are details that could potentially be viewed. This type of awareness helps create a more intuitive interface and reduces user frustration. The details of relevance are the kinds of extra information that could be included in a story. Details may include acceptance criteria, graphs, multimedia, documentation, web links, history, dependencies and more. Also when zooming in, users should be made aware of the ability to pan and in which directions they can pan to reveal the hidden content. Guideline: Always visually indicate the presence of hidden details that can be optionally displayed. 


\subsection{Exploring and Filtering Information}

When using a traditional physical Cardwall, there is very little that can actually be explored since the whole idea is to present a big picture of a work in progress. The digital Cardwall can be much richer and it can allow users to explore the project in several ways including the ability to view previous iterations, the backlog, and even other projects. When we further enhance the digital Cardwall with the ability to filter, we open the door for a query language capability. In our field study, the participants expressed their frustration with regards to difficulty of searching, filtering, and tagging using their existing digital Cardwalls. They also expected to be able see dependencies and relationships between stories, break down epics into smaller stories, easily be aware that a particular story came from the decomposition of an epic. Furthermore, the participants wanted to create sets that could be used as the data for a query. Based on this, the following guidelines have been identified.

Working Sets and Queries: The concept of a working set helps the designer to know where results can be pulled from when dealing with users who want to drill down and focus or find some piece of information. An appropriate starting point for the scope of the working set is to default to the contents of the current view. For example, when viewing the current iteration, the stories on the Cardwall together form the default working set. The main point of the working set is that it can be iteratively reduced, such that the result of a query becomes the input for subsequent queries. In this way, the query operations are composed semi-automatically. The ability to reduce the working set was identified as an important enhancement that the digital Cardwall could implement, but we are also aware that users will only take full advantage of this feature if it is easy to use. The point of reducing the working set is to help users focus and quickly find information that may not be obvious or visible. Finally, the users should be able to save and label the current working set so that it can be re-visited at a later point. Guideline: Allow users to create sets based on story details and further explore them via the composition of queries.

Query: The operations we need for reduction are a simple query language based on predicate calculus, with basic support for the unary "NOT" operator, the binary "AND" and "OR" operators, the for-all and for-each operators as well as parenthesis for changing the order 
of precedence. Queries can be used to ask complex questions like "Find all the stories that have been completed, but for which there were reported bugs.". Guideline: Make simple queries easy, but also support a rich set of query operators.

Custom Attributes and Annotations: The ability to apply attributes to artefacts allows users to filter the working set by including or excluding artefacts that share a particular attribute. Filtering can be performed before or after a query and should be dynamic such that the addition or removal of categories should automatically refresh the results of the working set to reflect the change. It should be possible to use named attributes directly in user queries.

The ability to annotate is really a specific instance of the more general case of applying attributes explained above. The literature review of physical Cardwalls found that teams often marked up their Storycards with meaningful symbols, however, we did not see this behaviour in our study because this feature was not supported by the digital Cardwalls being used. In our data there was evidence to support the use of custom attributes to mark different components, business goals, roles, people, epics, etc. However, since Agile teams have the flexibility to customise their individual processes and through the benefits of retrospectives their own process can actually evolve, it is reasonable to expect the need for other types of attributes, not currently envisioned by us or expressed in our data. Furthermore, our data does indicate that all attributes should be searchable and their use should be valid in queries. Guideline: Allow users to mark-up their Cardwall and the stories within it. Optionally, let them assign meaning to their annotations.

\subsection{Managing Backlogs}

Digital Cardwalls could be designed to easily switch between different views or different walls. A large display in a boardroom could be used to bring up walls from different teams and or projects. In our pilot study we observed how the team managed their backlog using a physical Cardwall that had stories separated by rows, where the top row contained the highest priority stories, the ones which were most likely to make it into the next iteration. The 2nd row had other high-priority stories, but ones that were of slightly less value than those in the first 
row. The rest of the backlog was found under the second row, and it was considerably larger then the other two rows and had all the remaining stories in no particular order. Similarly in JIRA, you can prioritize the stories in the backlog by moving them to the top or bottom of the view, you can also pre-assign version numbers as a way of ordering stories.

Just another View: You can think of the backlog as the starting point for planning sessions. All of the elements described in the guidelines found in this chapter also apply to the backlog. Guideline: Generally support all Cardwall view operations on the backlog as well on the current iteration.

Backlog View: The backlog is special and there are certain interactions which are specific to it. The backlog is often not very visible in projects but we found that the backlog is the starting point for every iteration planning meeting. Planning iterations involves many tasks including looking at and discussing the stories in the backlog. Guideline: The backlog needs to have its own view which can be easily shown or hidden on demand.

Prioritize and Estimate: The most important stories in the backlog are selected for the next iteration. Teams need to be able to prioritize the stories and clearly see the most important ones.

The ability to group stories in the backlog makes it possible for teams to loosely plan further into the future, including the ability to define the set of stories with the next highest priority which can be used if the team finishes an iteration early. In our pilot study, rows were used to group the stories of similar priorities. In our field study, some teams used colours to indicated stories in the backlog that could potentially be pulled if needed. Similarly, columns could be used instead of rows or colours.

Once the highest priority stories have been identified, teams need to estimate the amount of work each story will take. The estimation process is critical and serves as a way to scope the next iteration. When the highest priority stories have been estimated an agreement has been reached as to the scope of the next iteration, it should be easy to officially activate or start the iteration. Guideline: Support flexible prioritization of stories.

Not just Stories: We saw in our study that sometimes teams put more than stories in 
the backlog. They also include information like the availability of the team members and their schedules and vacations. They can also include artefacts that represent work needed to address technical debt ("Gold cards"). This information helped to keep their estimates and expectations grounded and accurate. To satisfy this need, it may suffice to consider using a more general artefact with a designated "type". Stories may be the most common "type" of artefact and should be the default type, however, the ability to give an artefact a type provides flexibility and modularity. Type assignation should be available on creation of the artefact but also after the fact. New artefacts should automatically be added to the backlog. Guideline: Support the addition of diverse artefacts in the backlog, not just stories.

Relationships: There is a natural, hierarchical structure that can be captured by thinking of a project as the composition of releases. Often, apart from the continuous releases that occur after each iteration there is also the concept of an official 'release', which is more like a milestone. A release signifies the completion of a significant portion of work. In this way, projects are composed of releases, and each release is composed of iterations.

Another kind of relationship is one that represents epics. While planning iterations and discussing stories, it is often the case that stories are identified as being too general, or too large in scope for one iteration. When this happens the story may be considered an epic and the team may wish to break it up into new, smaller, more manageable stories, which the team should be able to complete in one iteration. A hierarchical structure is also created when epics are decomposed into smaller stories. The ability visualize the hierarchical structure and the ability to traverse the hierarchy in both directions is important and increases the user's ability to explore.

Stories can also have an associative relationship. Stories can be associated with other stories, for a wide range of reason, often particular to the domain or the specific project. We saw in our study that it would be useful to allow users to create links between artefacts. Users should be able to use association to quickly jump from one artefact to another.

Dependencies exist when the functionality of one story requires that the functionality 
of another story be already implemented. The ability to mark a story as dependent on another is important, as is the visualization of the dependencies. The satisfaction of this requirement will help ensure that the stories being added to an iteration do not depend on the functionality of stories yet to be implemented. Guideline: Support traversal of the hierarchical nature of the releases and iterations to provide automatic relationships, and also support user customizable relationships.

\subsection{Multi-disciplinary use of Stories}

One advantage of the physical Cardwall is its simplicity. The Cardwall can be easily customized to meet the needs of the team using it. One particular customization we found important involves the various roles of different team members. We speculate that individuals in non-developer roles (such as interaction designers or testers) will see the value in digital Cardwalls if their work is made distinct on the Cardwall. We foresee that such a development will add new linking and customization requirements. Cardwalls can be customized to align with virtually any linear process or workflow, which is why they are ideal for supporting different types of teams, not just developer teams or multidisciplinary teams.

The following scenarios help to frame our guidelines for implementing a digital Cardwall that supports traditional developer teams, but also multi-disciplinary teams and nondeveloper teams. In the first scenario, a team of interaction designers might work on stories from a variety of projects simultaneously. In contrast to the situational awareness needs of developers, designers need to see whether or not the stories have been 'designed', i.e., whether or not there is a corresponding UI, story, sketch, or wireframe, whether they have tested the stories with a variety of scenarios, or whether they have tested the stories with their customers. This suggests very different columns for Cardwalls used by teams of interaction designers. Also, the artefacts on a Cardwall of this nature would come from multiple projects and therefore exists on distinct Cardwalls.

Another scenario might be a team of testers whose job is to convert a story's acceptance criteria into executable tests. A team like this might also be responsible for creating tests across many projects, so their backlog would pull stories from the backlogs of other projects and the stories they complete could be the source (the backlog) of stories for the next iteration.

Cardwalls can also be valuable tools at a personal level. In this situation, the story 
someone is working on could be broken up into tasks. Each task could be treated as a story, including estimates, and as tasks are completed the overall progress of that story on the team Cardwall can be updated to show the progress.

Role Swimlanes: One common way of visualizing the distinct roles in a project is through swimlanes. Swimlanes help visually separate the flow of stories across the columns of a Cardwall, where rows can represent the lanes and the stories in each lane are independent and can therefore be worked on in parallel to the stories in other lanes. This way, a multidisciplinary team of web-developers, designers, and back-end or infrastructure developers could share the same wall and the stories for each of these teams could be visually separated into swimlanes. Guideline: Stories associated with various roles should be visually identifiable and the use of swimlanes is ideal for this purpose.

Process Customization: One important reason that Cardwalls can be easily aligned with so many distinct processes is that teams can customize the columns to match their process. To seamlessly support virtually any team, the digital Cardwall must allow teams to add, remove and rename columns. This way each column on their Cardwall aligns with a distinct step in their process.

Another important customization option is the ability to customize and create new annotations, which would be re-usable and would allow teams to quickly identify artefacts that share the same annotations. This can be further enhanced by designing the system to differentiate between distinct annotations so that they can be used for querying, searching and filtering. Guideline: Allow teams to customize the Cardwall so that it aligns with their particular process.

Cross-Project Links: A Cardwall where the stories could be pulled from a variety of sources would help teams in a support role adopt the Cardwall. From the scenarios mentioned above, teams like QA, testing and designers would potentially fit this role and benefit from this ability. It is common for team members occupying roles of this nature to work simultaneously on different projects. From the perspective of Management it would also be useful to allow them to get an even bigger picture which might include multiple projects separated into swimlanes. Guideline: Support roles where team members' responsibilities extent beyond a single project. 


\subsection{Keeping the Cardwall Updated}

One important complaint that users had with their existing digital Cardwalls was that there were too many steps to accomplish what they felt should be seamless or at least easier than it was. At the top of their list was saving and refreshing their Cardwalls. Our participants felt that it was an annoyance to have to manually remember to save updates and to manually have to refresh their client to see changes made by others, which may or may not have even been saved. This was a recurring theme through our observations and our interviews, but really in terms of design guidelines this theme can be expressed succinctly.

Automatic Save and Refresh: Every action performed by users on the Cardwall should automatically be saved. There is no need to force users to do extra work just to confirm that they want to save their changes. A distributed system of this nature is necessarily backed by a database, and local changes should automatically be pushed in real-time. Of course, in a distributed environment, the possibility of conflicting changes must be dealt with and default behaviours defined. In general, a conflicting change should be sent back to the originating source (or sources) for manual resolution. Similarly, remote changes should automatically be sent to all clients. There is an important decision to be made regarding exactly when changes are pushed to clients. Changes could be handled by the database and successfully stored changes could be pushed to clients or the client from where the change originates could be responsible to communicate changes to all peers. This kind of synchronization is a well-explored issue in the field of computer supported collaborative work (CSCW). For example, see the work of Borghoff and Schlichter [12]. Guideline: Support automatic synchronization of Cardwalls; extra steps to save and refresh should be avoided.

Reduce Change Blindness: When a client receives an update, there needs to be some visual cue that draws the attention of the users so that the received changes become visible. In this way, the instances of change blindness will be reduced when teams are collaborating remotely.

The goal of such a system should be to support situational awareness. Support for situation awareness will also help to reduce the potential for conflicts between actions initiated by distributed users. Careful consideration must be paid to the issues of 
latency, network flooding and how they relate to the system's ability to support a high level of awareness across all users. A balanced approach should be taken so that there are sufficient cues about what other remote users are doing, such that it would be hard to concurrently create changes which would result in a conflict at the database. In this way, an ideal solution should create an experience that balances these somewhat conflicting issues. Again, this topic is well explored in CSCW [12]. Guideline: Create awareness of remote changes to reduce errors and increase usability.

\subsection{Summary}

This chapter has presented some important guidelines for the design of a digital Cardwall, as well as, some best practice advice for end users. Table 5.1 lists the guidelines and outlines to what extent Trello and JIRA (with JIRA Agile plugin) support the features outlined in our guidelines. The first column contains the guidelines, organised by our themes. The second and third columns represents the extend to which a guideline is supported by Trello and JIRA respectively. We use ' $\mathrm{F}$ ', for fully supported, ' $\mathrm{P}$ ' for partially supported and ' $\mathrm{N}$ ', for not supported. To conclude the chapter we will briefly discuss some of the more interesting results found in Table 5.1.

In the first theme, 'Digital Cardwalls', we say the both Trello and JRA have partial support for simultaneous multi-user access, design for diverse screen sizes and direct touch. We say partial support for the following reasons, 1.2) multi-user access: they both support multi-user simultaneous access from different machines and or physical locations, but not from one co-located location, where multiple users may wish interact with with a shared Cardwall driven by a single machine. 1.3) They have partial support for diverse screen sizes because they both have mobile version, optimized for smaller devices, however, neither has an optimized design for large high-resolution displays. 1.4) They support direct-multi-touch in that, they have mobile version, however, as far we can tell they have no specific design to leverage advantages possible for co-located collaboration on larger touch-enabled surfaces.

In the 'Detailed Information' theme we indicate partial support for 2.2) Support zoom and pan because their mobile versions support the zoom feature, since the functionality is built into the mobile browser. Partial support for 2.4) indicate the presence of hidden details was given to both because they do provide visual cues indicating extra detail is available, 
however not all types of hidden information is supported. For example, there are no visual cues in JIRA to indicate the presence of hidden dependencies.

In the third theme, 'Exploring and Filtering', Trello does not support guideline 3.1 since it only supports basic searches. However, guideline 3.1) Allow users to create sets based on story details and further explore them via the composition of queries was partially supported at the time of the observations, however newer versions of JIRA now support this feature and they have even introduced JIRA Query Language (JQL) [6] which is similar to SQL and supports advanced querying. Guideline 3.2) Make simple queries easy, but also support a rich set of query operators is partially supported by JIRA, since users complained about this being difficult to use. New versions of JIRA have improved this with the introduction of JQL, however usability of this feature needs investigation. Trello partially supports this guideline since it supports simple searches, but not the rich queries that we envisioned when developing this guideline. For guideline 3.3) Allow users to mark-up their Cardwall and the stories within it both Trello and Jira partially support this. However with Trello, you are confined to a small set of options like adding coloured labels with specific names, unfortunately these labels are not searchable. In JIRA there is more sophisticated support for this but again, our participants complained about the usability of these features. Improvements have been made in JIRA that deserve further investigation.

For the 'Managing Backlogs' theme, the guideline 4.1) Generally support all Cardwall view operations on the backlog is partially supported by Trello, because it does not distinguish between boards or the artefacts with the boards. In Trello you can have a board dedicated for the backlog, or you could have the backlog be part of any board, the capabilities are the same, regardless. In JIRA the backlog is available as part of a project and supports a rich set of features which allow teams to prioritize, estimate, break down stories or epics and filter to help select the appropriate stories for an iteration. Trello partially supports 4.2) The backlog needs to have its own view which can be easily shown or hidden on demand, since the backlog could simply be a board all on its own. For JIRA, the backlog is a view that can be on display or not, giving JIRA full support for this guideline. Trello partially supports 4.3) Support flexible prioritization of stories since it allows stories to be moved around within a column, such that the highest priority stories could be found at the top of the backlog. In JIRA, there more sophisticated support for this guideline, and it even allows for planning any 
number of iterations in advance. For guideline 4.4) Support the addition of diverse artefacts in the backlog, not just stories Trello is extremely flexible in terms of the contents of a card and this means you could attach almost anything to a card. JIRA is more limited with what you can see in the backlog and only supports a subset of issues (stories). For the last guideline 4.5) Support traversal of the hierarchical nature of the releases and iterations to provide automatic relationships, and also support user customizable relationships Trello allows you to get links to each card and include those links in other cards, however they can not be used to quickly move from one card to another. In JIRA, there was partial support for this guideline, however newer versions have improved support for this and should be investigated further.

In the 'Multi-disciplinary use of Stories' theme, Trello only partially supports 5.1) Stories associated with various roles should be visually identifiable because you can use coloured labels for this purpose. In JIRA, users can use swimlanes, colours and labels to support this guideline. For guideline 5.2) Allow teams to customize the Cardwall so that it aligns with their particular process, the simplicity of Trello allows it to easily align with virtually any process which is why it has full support. At the time of the observations, JIRA did not support this but now, the ability to add and customize columns has been introduced and it can now align with different process. The usability of this new feature should be investigated further. By default Trello searches will search across all Cardwalls and thus partially supports guideline 5.3) Support roles where team members' responsibilities extent beyond a single project. Support for this feature in JIRA is now available with JQL, but may not have been available at the time of our observations or simply difficult to use.

Finally, for the sixth theme 'Updating the Cardwall' Trello partially supports 6.1) Support automatic synchronization, however there are many interactions that still require the user to confirm before the action is made available to other remote views (like the creation of a new card). In JIRA, there is a much more complicated full featured system, there are still requirements to manually save, and requirements to refresh in order to view remote updates. For the last guideline, 6.2) Create awareness of remote changes to reduce errors and increase usability both Trello and JIRA partially support this guideline by allowing user to subscribe to changes to stories. Trello also has a notification area where changes are posted. Similar features are available in JIRA, however some of our participants found the email notifications 
overwhelming. 
Table 5.1: Summary of the guidelines and support from them in Trello and JIRA with JIRA Agile. (F: full support, P: partial support and N: not supported)

\begin{tabular}{|l|c|c|}
\hline Guidelines by Themes & Trello & JIRA Agile \\
\hline 1) Digital Cardwalls & & $\mathrm{F}$ \\
1.1) Always support a large format overview of the entire project to facilitate & $\mathrm{F}$ & $\mathrm{P}$ \\
team and stakeholder discussions. & $\mathrm{P}$ & $\mathrm{P}$ \\
1.2) Allow simultaneous multi-user access. & $\mathrm{P}$ & $\mathrm{P}$ \\
1.3) Support diverse screen sizes with appropriate design. & $\mathrm{F}$ \\
1.4) Support direct multi-touch where possible. & $\mathrm{F}$ & $\mathrm{P}$ \\
\hline \hline 2) Detailed Information & $\mathrm{P}$ & $\mathrm{F}$ \\
2.1) Always begin with and default to a general overview display. & $\mathrm{F}$ & $\mathrm{P}$ \\
2.2) Support zoom and pan to allow users to leverage diverse displays. & \\
2.3) Allow optional display of details that would otherwise clutter the current \\
view. \\
2.4) Always visually indicate the presence of hidden details that can be op- \\
tionally displayed. & $\mathrm{P}$ & \\
\hline \hline 3) Exploring and Filtering & & $\mathrm{F}$ \\
3.1) Allow users to create sets based on story details and further explore them & $\mathrm{P}$ & $\mathrm{P}$ \\
via the composition of queries. & $\mathrm{N}$ & $\mathrm{P}$ \\
3.2) Make simple queries easy, but also support a rich set of query operators. \\
3.3) Allow users to mark-up their Cardwall and the stories within it. Option- \\
ally, let them assign meaning to their annotations. & $\mathrm{P}$ & \\
\hline
\end{tabular}

4) Managing Backlogs

4.1) Generally support all Cardwall view operations on the backlog as well on the current iteration.

4.2) The backlog needs to have its own view which can be easily shown or hidden on demand.

4.3) Support flexible prioritization of stories.

4.4) Support the addition of diverse artefacts in the backlog, not just stories.

4.5) Support traversal of the hierarchical nature of the releases and iterations to provide automatic relationships, and also support user customizable relationships.

5) Multi-disciplinary use of Stories

5.1) Stories associated with various roles should be visually identifiable and the use of swimlanes is ideal for this purpose.

5.2) Allow teams to customize the Cardwall so that it aligns with their particular process.

5.3) Support roles where team members' responsibilities extent beyond a single project.

6) Updating the Cardwall

6.1) Support automatic synchronization; extra steps to save and refresh should be avoided.

6.2) Create awareness of remote changes to reduce errors and increase usability.

\begin{tabular}{|c|c|}
$\mathrm{P}$ & $\mathrm{F}$ \\
$\mathrm{P}$ & $\mathrm{F}$ \\
$\mathrm{P}$ & $\mathrm{F}$ \\
$\mathrm{F}$ & $\mathrm{N}$ \\
$\mathrm{P}$ & $\mathrm{P}$ \\
& \\
$\mathrm{P}$ & $\mathrm{F}$ \\
$\mathrm{F}$ & $\mathrm{P}$ \\
$\mathrm{P}$ & $\mathrm{P}$ \\
\hline
\end{tabular}




\section{Chapter 6}

\section{Interacting with Surface Technologies}

Given the importance of physical interaction with Cardwalls, we were excited about the new interaction possibilities afforded by the use of multi-touch capable surfaces. The cost of large, high-definition displays continues to fall, and plug-and-play multi-touch frame-overlays have become available. These frames can convert standard flat screen televisions into fully capable multi-touch displays. We believe that a large digital multi-touch enabled Cardwall would have a clear advantage over existing digital walls and re-purposed issue trackers. However, interaction design for such displays is in its infancy.

The remainder of this chapter presents the results of an extensive literature review. We focused on general interaction issues that arise when interacting with multi-touch enabled surfaces and, in particular, large displays that support multi-touch.

We identified six interaction issues which we will briefly introduce in terms of their importance to potential user interactions with digital Cardwalls on a large direct-touch displays. In the final section entitled 'Issues Arising for Digital Cardwalls' we describe how the identified issues can be addressed in the interaction design of a digital Cardwall.

Collaboration for Surface Interactions: Communicating the state of the current iteration is one of the key roles played by the Cardwall. When the Cardwall is used as recommended, it is on constant display and situated close to the team. It becomes a gathering place where impromptu conversations about the project and the stories being worked on are discussed. Everything about the Cardwall and the supporting environment is highly collaborative. Multi-touch technology allows us to step away from the single user interaction paradigm and opens the door for supporting truly rich collaborative interactions.

Pointing and selecting: Pointing is one of the most common forms of non-verbal communication. Its use is inevitably present in most discussions, but particularly when using the Cardwall. It is often the case that attention should be drawn to a particular story 
and pointing is a natural way to communicate this. Selecting is also an important task, especially when planning iterations. The ability to select multiple stories for the purpose of planning an iteration is an important recurring event.

Gesturing: Similar to the previous two issues, gestures are a naturally occurring non-verbal form of communication. Creating natural gestures for the exploration of the backlog or relationships between stories seems like a good use of gestures for the Cardwall.

Hovering: In traditional GUI systems, hovering is a common form of interaction that allows users to preview without committing to a particular action. For Cardwalls, the idea of a gesture which allows users a sneak peek of some information is intriguing and could potentially be used in a 'what if' scenario when planning iterations and selecting the stories for those iterations.

Text: Virtual keyboards require time to get used to, but they are ideal for the amount of text needed for creating a typical story. However we need to understand the limitations and opportunities for text input.

Identity of the interactor: In co-located meetings using physical Cardwall, it is easy to know which team member is responsible for changes. With a digital Cardwall we can try and create that same awareness even when meetings involve distributed team members.

\subsection{Collaboration for Surface Interactions}

Buxton has emphasized that, at a minimum, the primary task of collaboration should not be impeded by technologies designed to support them [38]. Ideally, collaborative technologies should enable workflow.

Scott [59] was also concerned about workflow when she created the following guidelines for designing collaborative systems for tabletops. Her guidelines assert that technology should:

- support interpersonal interaction

- support fluid transitions between activities

- support transitions between personal and group work 
- support transitions between tabletop collaboration and external work

- support the use of physical objects

- provide shared access to physical and digital objects

- consider the appropriate arrangements of users

- support simultaneous user actions

Workflow can be achieved through well-designed applications that support collaborative tasks, but workflow is also impacted by the minutiae of interactions with surfaces because these can distract from the primary purpose of collaboration. Therefore designing for collaborative tasks demands a lot of attention to the mechanisms of interaction because these have an enormous impact on flow. A vast amount of research has been directed toward enabling workflow by developing, testing and comparing interactive techniques. These are the primary challenges within the field at the moment because a gap remains between existing and ideal mechanisms for interaction.

Enabling workflow was the strongest argument made in Lee et al.'s recent paper on the importance of providing interactivity when working with visualizations [40]. Lee et al. claim that the information visualization community has made limited use of novel input devices and the field of human-computer interaction to advance the work of information seekers. They argue that visualizations are an aid to making sense of data and to thinking, that interactions allow us to arrive at the right visualization that enables exploration and refinement of views and understanding, and that interaction is essential to enabling analytic discourse through which knowledge is constructed. Linking this to the topic of interaction design, the question then becomes, which interaction model is most effective? The authors draw us towards considering models that impose the least cognitive load on users, allowing more focus on exploratory tasks and less on interface elements.

Moving away from the direct manipulation paradigm, which includes models like WIMP (windows, icons, mouse and pointer), instrumental interaction, and proxemic interaction, Lee et al. point to other broad paradigms as potentially more beneficial to users of visualizations including reality-based interfaces, surrogate interaction techniques and NUIs (natural user interfaces), all of which have been carefully defined in their article [40]. We pursue the 
latter model of interaction in this chapter, while also drawing on some good ideas from the direct manipulation paradigm. The NUI model of interface design for surfaces is described by Wigdor and Wixon and made possible by pen, touch, gesture, speech and sketching input mechanisms [68]. For very good descriptions of the other interface models, refer to Lee's paper [40].

Collaborative information exploration is not just about exploring or sharing visualizations, but can involve using raw data and many other types of information such as text, statistics, video, photos and maps. We feel that an exploration of the NUI model, that aims for an interface that is effectively invisible to its users and is based on nature or natural elements would help. It could direct design efforts towards creating interfaces for surface technologies that would allow users to manipulate digital artefacts on surfaces as they would manipulate tangible objects, i.e., in natural ways. We feel this would support collaborative work across a broad spectrum of tasks.

This chapter looks closely at advances in some of the interaction mechanisms that tend to emphasize a more natural form of interaction and allow users to focus on the task at hand and not on manipulating icons, menus, or instruments that are essential elements of other interaction models. In the language of Lee et al. [40], NUIs provide more "degrees of freedom" so that there is a greater link between user intent and execution (i.e., exploration of data) which in turn enables workflow.

This section describes how the state of the art in interaction techniques for surface technologies is advancing.

\subsection{Pointing and selecting}

Indexical communication involves pointing, which is ubiquitous in collaborative work. Sometimes pointing is used to draw the attention of others; however, sometimes pointing is a means of interacting with the system. When pointing on regular displays (with your cursor or finger), no interactive event is triggered. However, when using a touch display, an innocent indexical event can cause unintended system responses (e.g. pointing could be misinterpreted as a click by the system if the user touches the surface). In this subsection, we discuss this and other issues that relate to pointing and how pointing can be supported. 
When interacting with large displays, the question of reach often arises. Due to the physically large nature of the display, artefacts may be out of reach. Sometimes in a collaborative scenario, this problem might be solved by increased interaction between the collaborators (i.e., asking for something to be passed to you), which could lead to better shared awareness and better engagement, but sometimes this solution falls short or is simply not feasible. For example, imagine a 10-foot high, wall-sized display where no one can reach an artefact near the top of the screen. The ability to pan the screen could be useful for accessing out-of-reach artefacts, but this would disrupt other users working on different areas of the display. The solution to this problem is the subject of ongoing research that focuses on methods to extend a user's reach beyond their physical limits. We present a variety of solutions.

Shoemaker et al. used the embodiment of the user in the form of a translucent virtual shadow that is cast upon the surface using a virtual light source. This shadow follows the user's movements. The angle of the shadow can be adjusted to allow the user to interact with the interface using their shadow, without every having to actually touch the screen [62].

Banerjee introduces Pointable, a cursor-based solution that is controlled using in-air bimanual gestures while wearing fiducial gloves. In this system, the dominant hand controls the selection and movement of a remote object, while the other hand can execute tasks, such as rotating the object [7].

Marquardt et al. unified the space between surface gestures and mid-air gestures (also employing fiducial gloves), so that they can easily transition between direct-touch actions on displays and in-air movements. As part of this work, they introduced raycasting gestures, a technique that seamlessly integrates pointing above the table and direct-touch manipulation of a selected object. Used in the opposite sense objects can be moved out of reach [42].

McAdam and Brewster designed an experiment to gauge the usability of interacting with a remote tabletop surface using a mobile phone [44]. They designed a system to test how well users could select and manipulate a dial and set it to a specified position. In one of three conditions the mobile phone duplicated the interface on the tabletop, and allowed the users to manipulate the tabletop interface indirectly using their phone. Their results were promising and they concluded that the phone as an input device for pointing and selecting had the best accuracy of all the techniques they studied.

A problem with pointing is that it is difficult to be precise. This problem regularly 


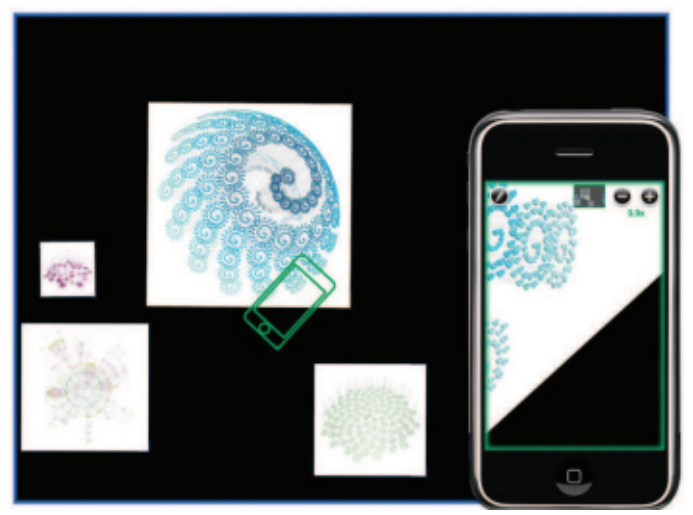

Figure 6.1: Selecting high resolution items with a low resolution pointing device. A solution to the "fat fingers" problem encountered when using tabletop displays. A smart phone displays an enlarged image of a part of the tabletop display. The user selects a part of the image by touching the smart phone display. Source: [65]

arises with applications designed for multi-touch surfaces. For high-resolution visualizations and collaborative work, one could imagine that fingers could lack the necessary precision required for exploring data. In fact, they could hinder exploration by obscuring information. Addressing the problem of "fat fingers", the disparity between object images on high resolution displays, and using fingers as a pointing device for selecting parts of an image, Voida and colleagues developed i-Loupe and iPodLoupe, which are lenses used on tabletop displays. These lenses (a rectangular green lens is shown in Figure 6.1) create a focus area where a magnified image of an object appears on a person's smartphone display, making it possible to select parts of it [65]. An alternative solution to the precision problem for multi-touch systems would be a surface system that could also process input from multiple styluses.

Sometimes what the user wants to indicate is an area or a collection. This type of selecting is typically a prerequisite to another action, such as a move.

Bier et al. introduced the idea of using Toolglasses and Magic Lenses [11] for surface applications. These are transparent widgets that are dragged around the surface to reveal more detail about the objects underneath. This idea has been used to solve a variety of interaction issues including selection [16] [2] [56] [55].

In a similar vein, Appert introduced three techniques for focus + context lenses that combined the ability to zoom and precisely select small targets in the focus area [2]; and Kaser created FingerGlass [37] a bi-manual lens that allows precise selection within a zoomed 


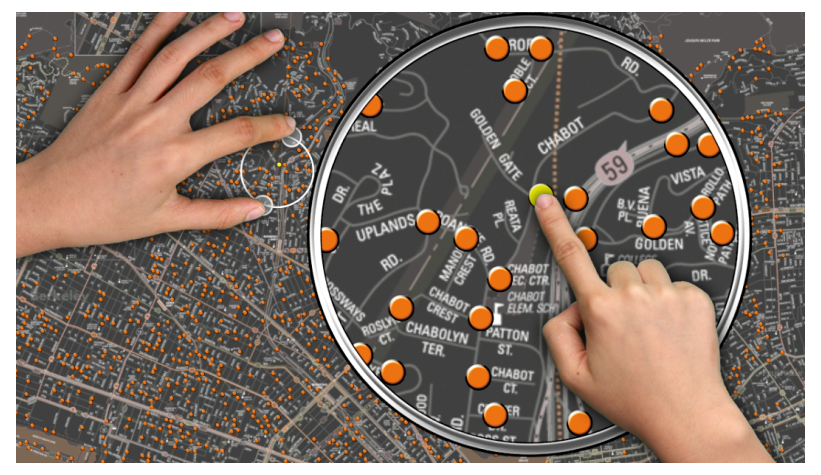

Figure 6.2: FingerGlass a widget for interacting with large displays. The FingerGlass widget helps users see more detail and helps with precise selection. Source: [37]

area while maintaining the context of the focus area. Figure 6.2 illustrates how one hand activates the widget and maintains context while the other hand is free to interact with the area of interest.

Other solutions to selecting on a direct-touch device involve reducing problems of occlusion. Sometimes the objects users are trying to interact with are small and may be hidden completely or in part by fingers or hands which are in contact with the screen. If a single touch could trigger an enlarged view of the small items in a specialized area, then users could interact with those items there.

\subsection{Gesturing}

Gestures introduce a novel method of interaction with touch-enabled surfaces. An understanding of designing gestures is at the heart of the NUI interaction model and so we spend some time describing some useful taxonomies and theories that could be used to design gestures.

Wobbrock et al. identified form, nature, binding, and flow as dimensions in their taxonomy of surface gestures shown in Figure 6.3 [70].

The form of a gesture describes its shape, at any point in time, and its path. If the shape of a gesture remains the same throughout movement over the display then it is static. If the shape changes then it is dynamic. The gesture is considered to have a path if there is movement other than simply changing the shape. For example, the dragging gesture shape is simply a finger blob; the gesture has a path because you move your hand across the screen, 


\begin{tabular}{|l|l|l|}
\hline \multicolumn{3}{|c|}{ TAXONOMY OF SURFACE GESTURES } \\
\hline \multirow{4}{*}{ Form } & static pose & Hand pose is held in one location. \\
\cline { 2 - 3 } & dynamic pose & Hand pose changes in one location. \\
\cline { 2 - 3 } & static pose and path & Hand pose is held as hand moves. \\
\cline { 2 - 3 } & dynamic pose and path & Hand pose changes as hand moves. \\
\cline { 2 - 3 } & one-point touch & Static pose with one finger. \\
\cline { 2 - 3 } & one-point path & Static pose \& path with one finger. \\
\hline \multirow{4}{*}{ Nature } & symbolic & Gesture visually depicts a symbol. \\
\cline { 2 - 3 } & physical & Gesture acts physically on objects. \\
\cline { 2 - 3 } & metaphorical & Gesture indicates a metaphor. \\
\cline { 2 - 3 } & abstract & Gesture-referent mapping is arbitrary. \\
\hline \multirow{5}{*}{ Binding } & object-centric & Location defined w.r.t. object features. \\
\cline { 2 - 3 } & world-dependent & Location defined w.r.t. world features. \\
\cline { 2 - 3 } & world-independent & Location can ignore world features. \\
\cline { 2 - 3 } & mixed dependencies & World-independent plus another. \\
\hline Flow & discrete & Response occurs after the user acts. \\
\cline { 2 - 3 } & continuous & Response occurs while the user acts. \\
\hline
\end{tabular}

Figure 6.3: Wobbrock et al.'s Taxonomy of Surface Gestures. Source: [70]

but while this happens the shape remains the same, it is always a finger blob. The pinching gesture's shape is dynamic because the two finger blobs are increasingly closer together, but the pinching gesture has no path because your hand does not move as you pinch.

The nature of a gesture is captured by four subcategories: symbolic, physical, metaphorical and abstract.

- Symbolic gestures are representations of things users can draw to trigger an action, such as drawing a question mark to activate help.

- Physical gestures are used to manipulate objects on the display.

- Metaphorical gestures are understood in terms of a user's mental model, and often the gesture represents an action. For example, walking your fingers across the screen could be the walking gesture in a game.

- Abstract gestures encapsulate all other gestures that do not fall into the three categories listed above. An example would be using a four finger tap to return to a starting point. 
Abstract gestures can be particularly difficult to design and they can be hard for users to learn and remember [36].

The binding dimension is primarily about the location of the gesture and its context or environment. This dimension of a gesture is categorized as: object-centric, world-dependant, world-independent and mixed.

- Object-centric gestures operate in a localized context, and they usually interact directly with or create objects. For example, the pinch gesture re-sizes an object to create a visualization of the same object only differently sized.

- World-dependent gestures are defined with respect to the real-world display environment. For example, dragging an object off-screen could move it into an imagined trash can in the real world.

- World-independent gestures require no information about the display environment, and generally can occur anywhere, except that the initial part of the gesture must not be performed on an object that can be manipulated by other gestures. A gesture to return to the desktop from any application is a good example.

- Mixed dependency gestures are simultaneously world-independent and either worlddependent or object-centric. A good example of this would be a rotation gesture where one finger from each hand is used. The first finger touches the desired object and acts as the point around which the object will rotate, while the other finger can be put anywhere else and are used to control the speed and rotation of the object.

The final dimension is flow. It describes a gesture in terms of what happens while it is being executed. A discrete gesture is one that must be executed entirely before anything happens on the display. An example would be a tap gesture to select an artefact, where the artefact is not selected until the gesture has completed. A gesture's flow is continuous if it requires constant recognition by the software. Continuous flow is required for the re-sizing gesture. As the user's fingers move closer together, the object becomes smaller, and as the user's fingers move further apart, the object gets bigger.

This taxonomy helps designers to understand the intricacies of gestures and can be used as an aid to software developers to structure gesture-recognition software. However, gesture 
design is far from a science and the above advice, directed towards designing a single gesture, is not clear enough to enable designers to easily associate a desired gesture with a suitable gesture type, nor does it provide guidelines for designing entire gesture sets.

Designing natural gestures is an open area where ongoing research is continuously producing new and better guidelines. Recently, many papers have found value in involving both users and experienced designers in the design of new gestures [52,70]. They have found that gestures designed using this user-centric technique produces gestures that are more natural and easier to remember.

A gestural interface for applications that support information seeking and exploration will necessarily have many abstract gestures to perform tasks where there is no adequate symbolic, physical or metaphoric representation. Examples of such tasks include, sorting, filtering, expressing a new hypothesis, and creating new paths of investigation. The design of gestures for these and other abstract concepts is even more challenging.

Hurtienne et al. introduced an important technique for designing abstract gestures that relies heavily on theories of cognition [31]. In their work they leverage the theory of image schemas and primary metaphors from cognitive psychology. Image schemes are knowledge about the world that is expected of a typical user. Everyone develops image schemas starting from their earliest interactions with the world as babies. Image schemas are developed based on experiences with the physical world, and they allow people to apply general concepts to multiple scenarios.

Up-down is an excellent example of an image schema. This is intuitively applied to many concepts like quantity or volume. For example, we understand without being told that as water is poured into a glass, the volume of water goes up or increases. Primary metaphors are the relationships between the image schema and the abstract concepts. When designing natural gestures, Hurtienne advises leveraging these primary metaphors whenever possible to express abstract concepts within the application. For example, a gesture based on an up-down image scheme could be created to increase or decrease the amount of information on the display. 


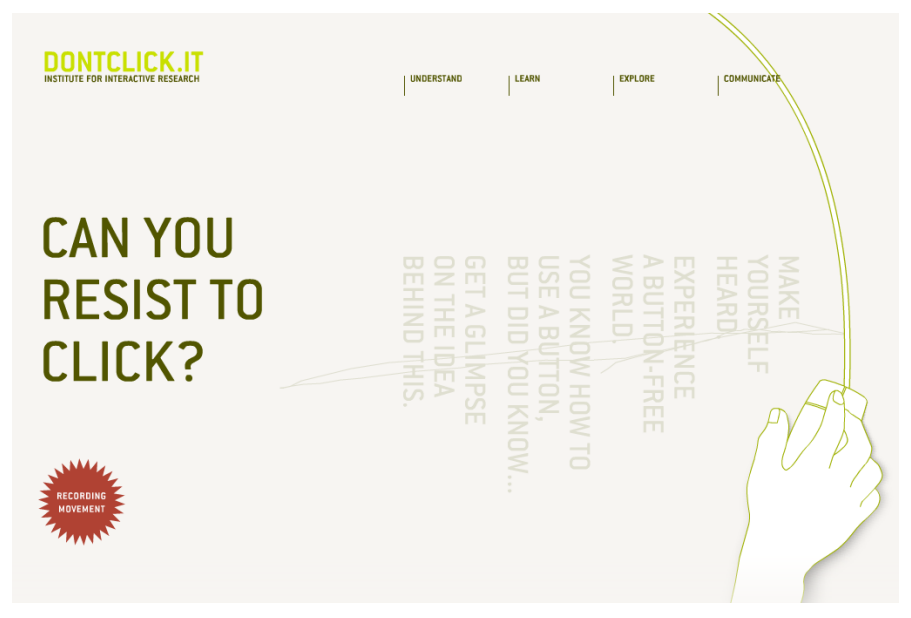

Figure 6.4: Hovering in web applications. A Website designed for clickless mouse interaction that could be used for surfaces. Try it at: dontclick.it

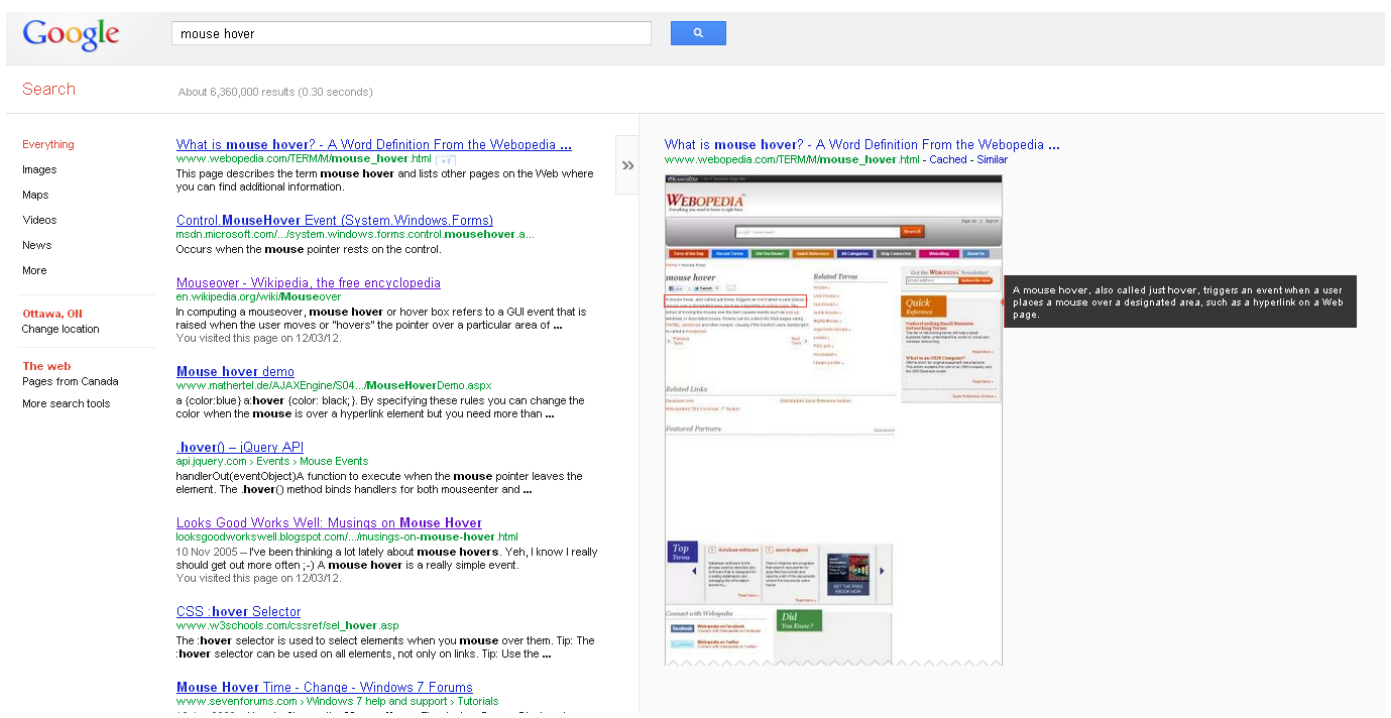

Figure 6.5: Hovering and Google Search. Hovering reveals a preview of search results. Source: www.Google.com 


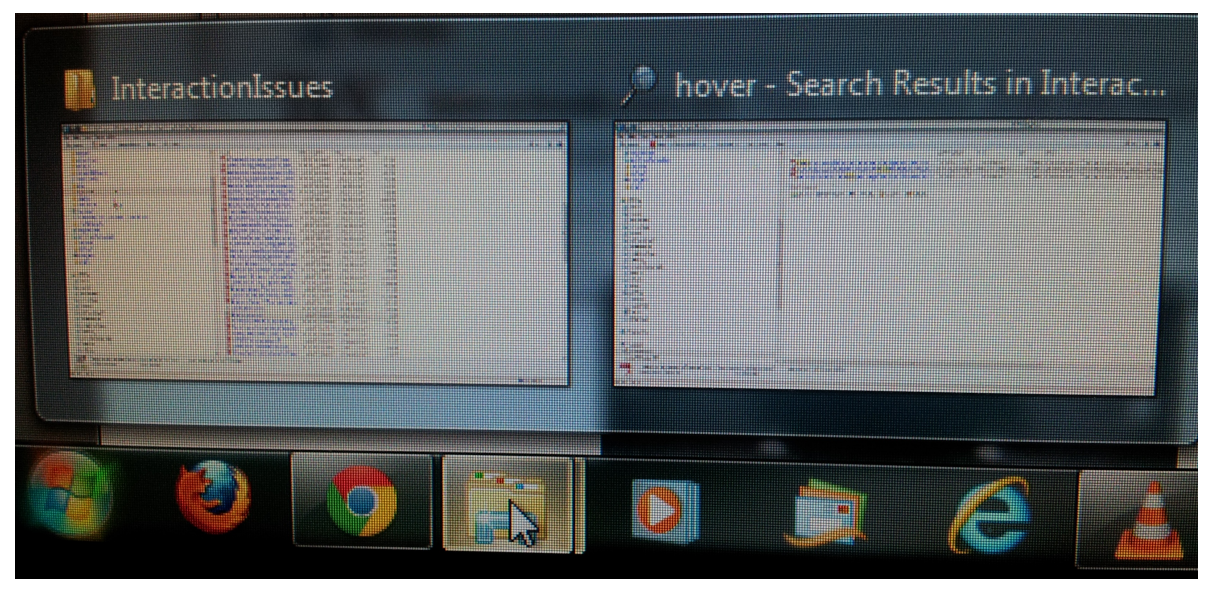

Figure 6.6: Hovering in Windows. Lingering the mouse pointer on top of the folder icon in a Windows taskbar to reveal a quick preview of open folders.

\subsection{Hovering}

Mouse-based systems provide different types of feedback and information about GUI elements when the user performs simple actions like placing the mouse pointer over top of a GUI element without clicking. Hover, or linger as it is sometimes referred to, is a specialized case of the mouseover or mouseout events that have existed since the first GUIs. At first these events were predominately triggered at the system level to change the appearance of the mouse cursor, thus cueing the user that further interaction was possible. Hovering has since been used to trigger tooltips or reveal information about an object. Today the use of hover is widespread and can be seen in all major operating systems from the Windows taskbar to the dock in Apple's OS X. Figures 6.4, 6.5, and 6.6 shows examples of how hovering is used in modern interfaces.

GUI elements often listen for mouseover events and respond by divulging hidden opportunities to interact with the objects. This is a light-weight approach that encourages discovery and loose engagement so that users quickly learn to explore an interface without the need to commit to any particular action.

Modern webpages also leverage hovering as an interaction mechanism. The website dontclick.it was designed specifically for mouse interaction minus the clicks [23]. This system highlights the benefits of hovering for effortless exploration. Recent research has also addressed the importance of hovering to determine users' intents while they interact with 
web search results [30]. This principle was applied by designers of Google's search engine so that hovering to the right of any search result item now reveals a preview of that result without requiring a visit to a web page.

The primary reason that hover, or the more general mouseover events, are difficult on direct-touch, mouseless systems is that direct touch requires physical contact with the surface, and this is a natural way to trigger an action. In general, touch-based systems recognize several types of interactions and different combinations of these interactions, but at the lowest level of the software (i.e., in the operating system), what this is often translated to is touch down, touch move and touch up events. How these events are interpreted and combined is dependent on the framework, the operating system and the application being used.

The seamless integration of touch and the operating system is still a work in progress and touch events are not handled in a consistent manner between systems or even within the same system. Research into how to port hover to direct-touch environments is ongoing and many potential solutions have been suggested.

Moscovich et al. proposed sliding widgets [47] as a replacement for traditional buttons in a touch interface. While they don't specifically address hovering, their design requires that a button is touched and dragged a small distance to trigger a "click". This therefore leaves a simple touch to trigger a hover event, an idea they demonstrated as workable in a word processor where a simple touch on an alignment button invoked an alignment preview. If the user then dragged the button slightly, the alignment setting was applied (and the button popped back into its original place). This solution helps avoid accidental activation and incorrect selection, which can be caused by the difficulties of precise selection on touch surfaces.

Using Bier et al.'s Toolglass and Magic Lenses technique for selection, it is also possible to design a hover lens that would trigger a preview of a target object. Dragging the widget above an object would trigger the hover event, just as if the mouse cursor had been placed above the object.

Another method for enabling hovering was proposed by Benko et al [9] who suggested that pressure could be used to differentiate between a hover and a click. In this research, pressure-sensitive devices were used to trigger a hover event based on the amount of force used when touching the screen. They simulated a pressure sensitive display with SimPress, 


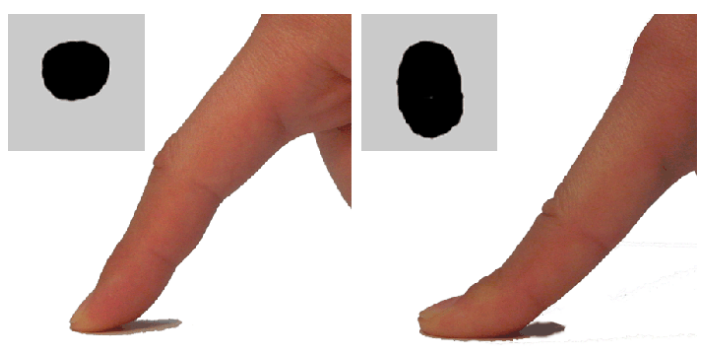

Figure 6.7: A solution to hovering on large displays. On the left a smaller finger blob represents a lighter touch and triggers a hover action. The right side shows a larger finger blob which in dictates a more forceful touch and triggers a click action. (The top left image in both sides show the shape of the blob detected by their system.) Source: [9]

where a rocking motion on a target object with a finger activates a listening state in a widget. Once activated, the system computes the touch area of the finger. A smaller area triggers a hover event, while a larger area triggers a click event, as shown in Figure 6.7.

Other solutions suggest the use of cameras to detect interaction above or in front of the surface. In this case, depth-sensing algorithms can detect an approaching finger or pen [69]. Pyryeskin developed a technique for vision-based multi-touch tables where they leveraged information cameras typically provide, other than the blobs that correspond to touches. This allowed them to detect several layers of hover space above the table [50].

\subsection{Text}

The ability for collaborators to simultaneously enter text while working around a large shared display is important for the efficiency of their workflow. In many scenarios the use of multiple physical keyboards is impractical for a few reasons. On a large horizontal display there is usually no good place to put the physical keyboards - they would potentially take up too much space and could occlude artefacts from view if they were placed on top of the surface. Similarly, on vertical wall-mounted displays, the question of where to put keyboards still exists. Currently there are two dominant areas of investigation to provide alternative solutions to physical keyboards, and both involve the use of virtual or soft keyboards.

One approach is the use of virtual keyboards designed for use on large interactive surfaces. Another is to investigate the use of mobile devices like smart phones and tablets which have 


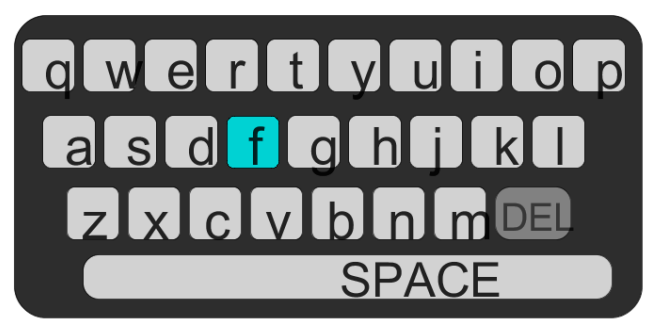

Figure 6.8: A simple QWERTY virtual keyboard. Source: [39]

their own virtual keyboards. They can be used for text entry in a more familiar way, since the use of virtual keyboards on handheld devices is increasingly common, while large interactive displays are only starting to make their way into real work environments.

Ko et al. investigated the size of the virtual keyboard, and whether or not the keyboards used on smart phones could be used equally well when ported to large surfaces [39]. They studied the usability of virtual keyboards in terms of speed and accuracy, but also considered space (how much screen real estate is used to display the virtual keyboard), occlusion (the effects of the user's fingers occluding the actual keys) and robustness (the tolerance for calibration errors as a result of older, lower resolution displays). The results of the study showed that the best performing keyboard was a more traditional QWERTY keyboard as seen in Figure 6.8. They also found that methods developed for mobile devices to help with the occlusion of the keys had little impact on the larger displays, even when the virtual keyboards were quite small compared to the screen.

Gesture Keyboard was introduced as a low cost prototype developed by Murase et al. [49]. With this system any built-in display camera or webcam can be used to detect typing on the surface in front of the display. Anywhere Touchtyping is another low cost alternative and was developed by Mujibiya et al. [48]; it uses an overhead DPL projector in combination with high speed video capture camera to detect typing on surfaces in front of a display using depth sensing algorithms. Figure 6.9 shows an example of both of these techniques.

\subsection{Identity of the interactor}

Often it can be useful for the application to be aware of its users' identities. This knowledge can be leveraged for access control of shared or personal artefacts, the activation of personal 


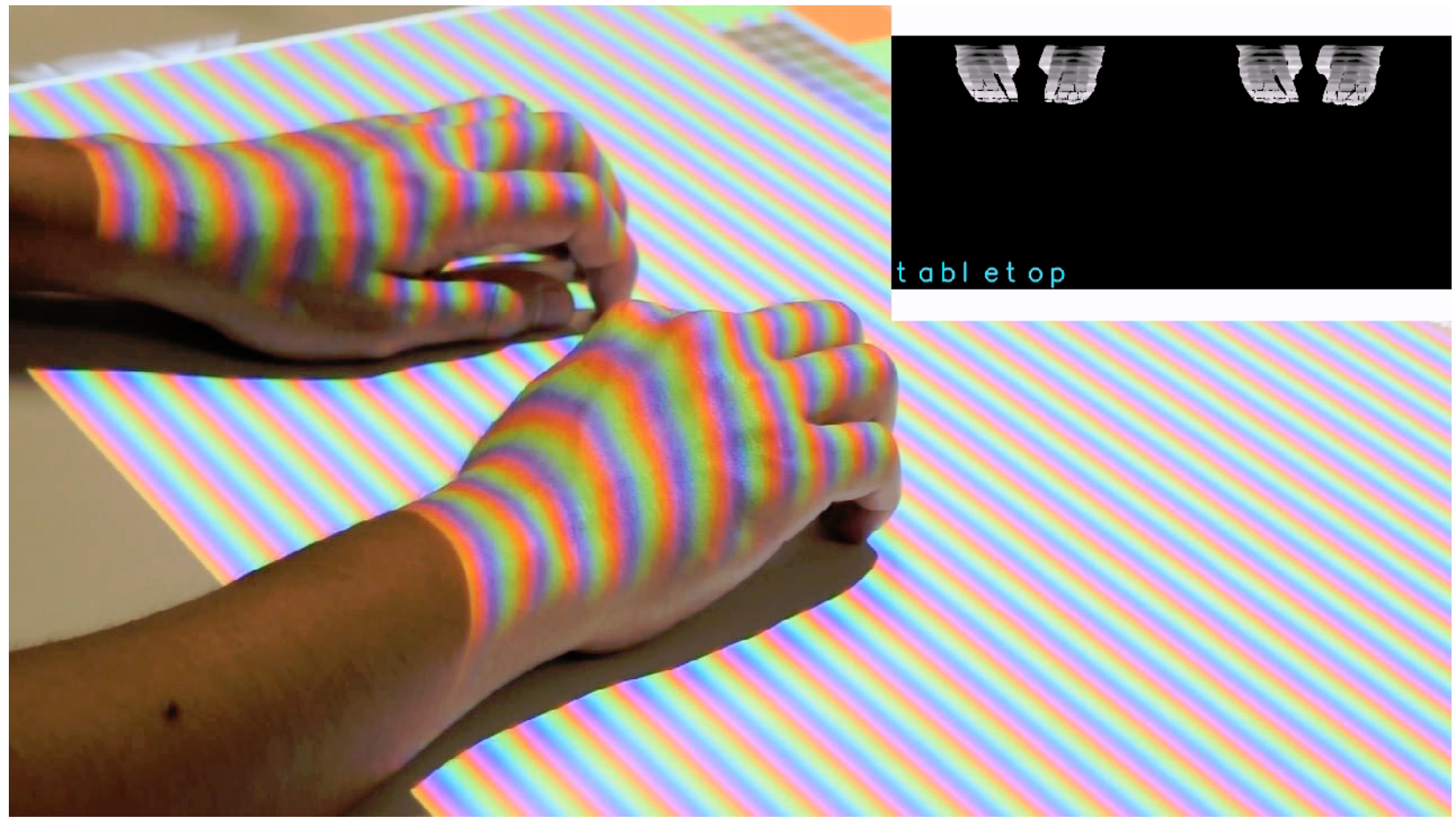

(a) Anywhere touch typing uses an overhead DPL projector and high-speed camera to detect typing on a surface. Source: [48]

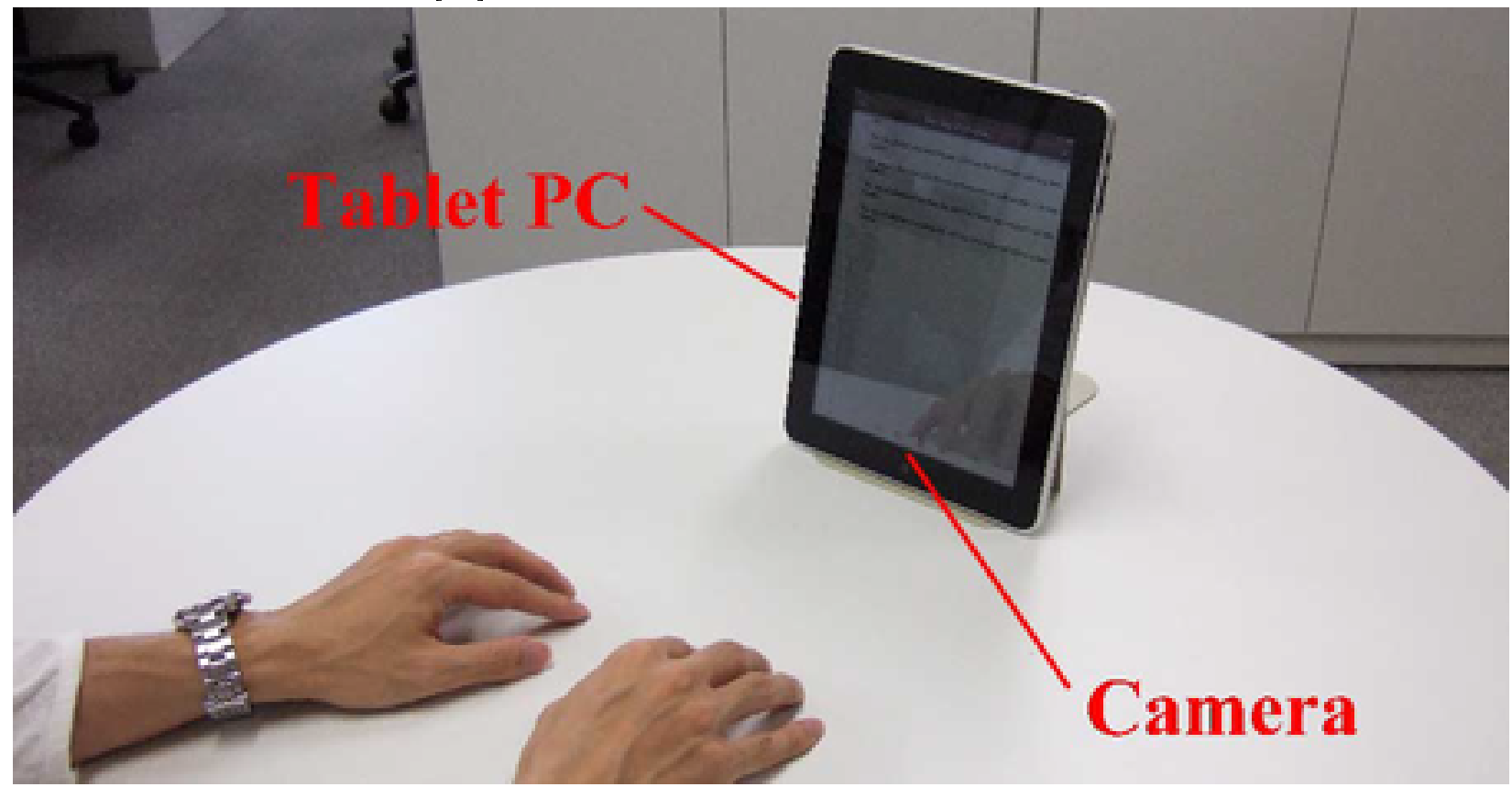

(b) Gesture Keyboard, a simple invisible keyboard, uses the built in camera or webcam to detect typing. Source: [49]

Figure 6.9: Text input on large displays. 
or private work areas, or to better understand the potentially complex interactions of multitouch and multi-user gestural interfaces. To clarify the latter point, imagine two users who are simultaneously interacting with a gestural-based interface. If each touch can be traced back to a particular user then the system's gestural recognition engine will be less likely to confuse the actions of separate users as a single gesture, and therefore it will be able to correctly identify gestures that are performed simultaneously by multiple users.

Recent work by Marquardt et al. developed the TouchID Toolkit, which was designed to facilitate the rapid development of touch aware applications through an event driven API [43]. It used fiduciary gloves to provide the system with user identity as well as hand or finger orientation and traditional touch and gesture recognition. In this system, each fiduciary tag on the glove could be assigned to specific tasks allowing quick access to a number of customizable tasks.

Schmidt introduced IdLenses [55], a widget that is invoked when a user places their hands down on the surface. It incorporated previous work called HandsDown [54], which was a system able to recognize a registered user based on the contour of their hand. Once a user is recognized, a re-sizable lens is used to create a private work area on the shared surface. Figure 6.10 shows a few uses of this novel lens. Information can be automatically entered in fields based on the user's ID. Comments can be seamlessly identified with their authors.

Sometimes hardware can provide partial solutions to the identity problem. The DiamondTouch table by Circle Twelve Inc. is a multi-user, touch- and gesture-activated surface that supports small group collaboration. An array of antennas embedded in the touch surface works in conjunction with a receiver that is connected to individual users (typically through their chair) to allow the table to distinguish between the touches of the various collaborators. This has significant benefits for avoiding misunderstandings by the software when many hands are on the display at once. Gestures can be interpreted easily if the system knows which multi-touches correspond with each other.

Pen-based systems like Anoto also aid with distinguishing between collaborators because each collaborator can have their own Anoto pen. 


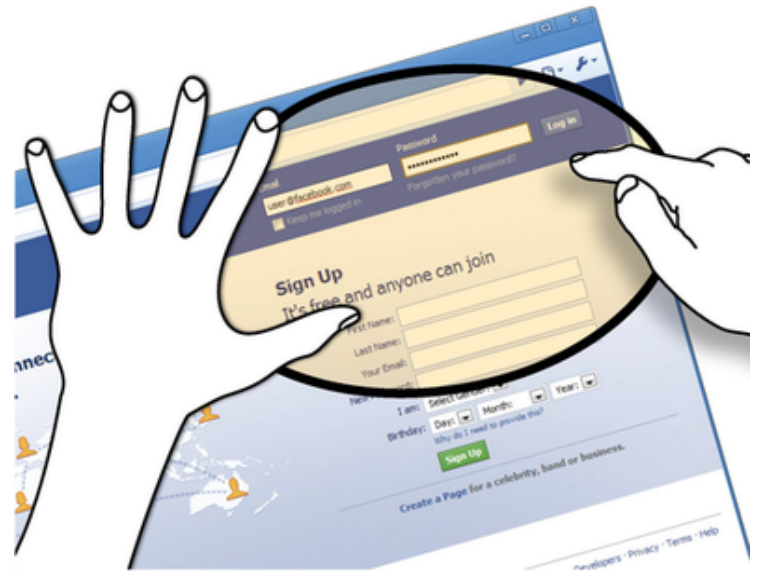

(a) Dragging the lens above a login screen allows automatic entry of login credentials.

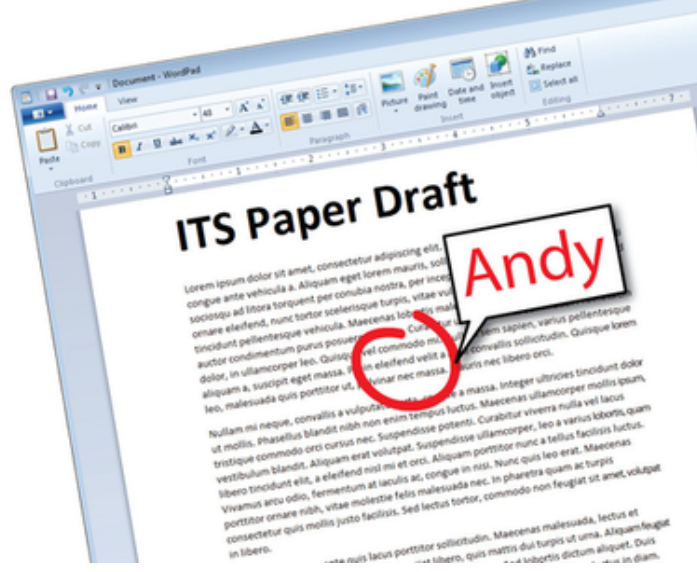

(c) An example of how the annotation looks when other users are viewing the document

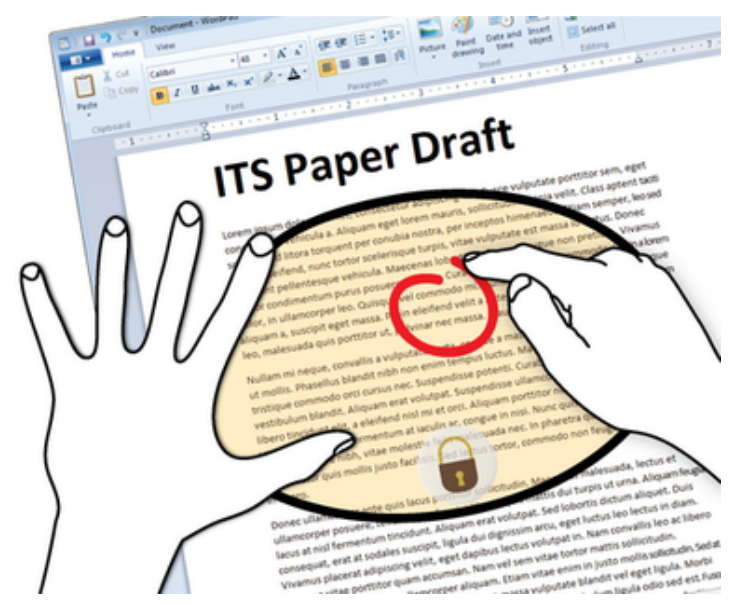

(b) Annotation of documents

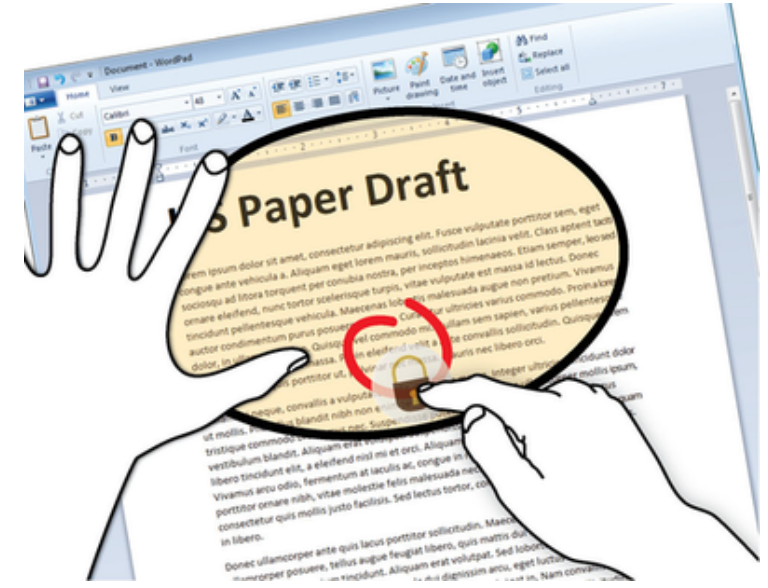

(d) Private annotations that are invisible to others can be made by drawing the annotation through the lock button. Source: [55]

Figure 6.10: A few examples of how IdLenses can be used. 


\subsection{Issues Arising for Digital Cardwalls}

Collaboration: Most multi-touch interfaces for large surfaces have support for simultaneous, multi-user interaction. Agile Cardwalls, generally involve the interaction from several teams members and often the discussion revolves around the details of multiple stories. Current digital Cardwalls do not support this type of interaction. Multi-touch Cardwalls will support this, but the design requires care to match the social process of the team.

Pointing and Selecting: Human communication is rife with indexical references, i.e., pointing, which involves verbally or physically indicating something. In artefact-rich environments such as Cardwalls, pointing behaviour is common and saves much time. Anecdotally it is well known that pointing, which is so easy on a singular small display with a mouse input controlling a cursor, becomes much more difficult when more displays are added, displays are tiled, or when the display becomes much larger.

Gesturing: The exploratory nature of planning iterations and discovering relationships could be ideally supported by a gesture-based query language. However, the design of the gestures and the language would be complex and it would require great care to make the gestures seem natural to the end user.

Hovering: As we discussed in the previous chapter, it is an uncluttered overview that is of primary importance. Hovering, or one of the described alternatives, could be very important and could be used to display details when they are required.

Text: Accomplishing serious work using a virtual keyboard can be frustrating and error prone. However, in the context of the Cardwall there is little need for text, other than for re-labelling columns, searches, and some Storycard annotations. Moreover, on a multi-user system, more significant text can be entered via a conventional keyboard.

Identity: In our study, meeting rooms were used for privacy and this could be enhanced even further with the addition of user identification. The individual identity of each participant can also be important and useful, especially to help increase later situational awareness in terms of who did what. 


\section{Chapter 7}

\section{Conclusions}

\section{$7.1 \quad$ Overview}

In this thesis we explored the topic of digital Cardwalls and Agile software development. We first reviewed the literature about physical Cardwalls and the Agile process, and then discussed existing digital Cardwalls (Chapter 2).

In Chapter 3, we presented a brief pilot study of an Agile team using a physical Cardwall. We used these findings to help design a field study described in Chapter 4 . This field study and the analysis constituted our main work.

Our field study involved the observation of 8 Agile teams using digital Cardwalls, involving 64 participants. From these teams we conducted interviews to delve deeper into what we observed. We used a qualitative thematic analysis approach to analyse our data and identified emergent themes. Our research questions concerned digital Cardwall practice and how well current solutions support Agile teams; we also wanted to identify specific opportunities to improve current solutions. We found that the tools being used by the participants in our study allowed the teams to successfully collaborate with distributed team members, and supported useful flexibility. However, the solutions did not help teams to easily identify or explore story relationships, especially in the backlog during planning sessions.

The identified themes from our field study were used to inform the design guidelines developed in Chapter 5. In the next chapter we presented a survey on principles for touch interaction on large surfaces, which we feel have great potential for digital Cardwalls.

\subsection{Discussion}

We believe that the guidelines developed in this thesis are strong and support for them would add real value to any digital Cardwall implementation. When viewed in isolation, each guideline attempts to address practical issues that address real problems that emerged through 
the analysis of our study data. In particular, we found potential value in the exploration and awareness of 'relationships' and the management of the 'backlog'. These are covered in the 'exploring and filtering' theme and the 'managing the backlog' theme and also in their associated guidelines. These two findings stand out as highly important and awareness of these themes and support for their corresponding guidelines in the design of a digital Cardwall will, in our opinion, bring the most value to the implementation. The next two subsections briefly recapture these themes.

We end the discussion section with the introduction of some limitations and conclude with ideas for future work.

\subsubsection{Relationships}

As the work in this thesis progressed, it became clear that the ability to explore relationships between stories was of critical importance. The work of Sharp et al. [60] foreshadowed the need to better support methods for enabling users to easily identify dependencies. They found from their Cognitive Dimensions analysis issues with hidden dependencies, viscosity and hard mental operations. The need to support the exploration of relationships and filtering of results was perhaps the most important finding from our study. This was especially important for the more experienced participants in our field study. When reviewing the principles for multi-touch interaction design we were struck by the important point made by Lee et al. [40] (discussed in Chapter 6) about the importance of interactive visualisations because they allow users to explore and actually refine the view until ultimately they arrive at the intended result. Moreover, Lee et al. describe this process as critical for facilitating deeper understanding and supporting collaborative discourse, which is exactly the point of the Storycard and the Cardwall in general.

\subsubsection{Backlog}

Traditional Cardwalls focus on the current iteration. For example, they feature columns of stories, where the column specifies the state of the story with regard to its implementation progress. However, the process of planning and selecting the stories for a particular iteration is much more about the backlog and its exploration. In our field study we found our participants frequently needing to explore and discuss stories within the backlog. It was important for 
them to identify dependencies between stories to enable the proper prioritization of stories. We speculate that the importance of the backlog has not been sufficiently recognized, since it is literally the entry point for Cardwall artefacts and a place where important conversations are begun.

\subsubsection{Limitations}

We reviewed the literature on physical Cardwalls and multi-touch enabled large displays. We also conducted a field study of digital Cardwalls, but we see the value in a more extensive study of both physical and digital Cardwalls, as well as further investigations into the design of a natural gesture set for large touch-enable displays. In particular, it would be useful to study a greater variety of Agile processes and software domains, with a larger diversity of teams and roles within them.

\section{Social Context}

Overall, Storycards and Cardwalls are excellent tools to support the planning and development of software projects. They are flexible and allow teams to use a customised process. The work of Sharp et al. [60] describes how the social context and social interactions are essential to the expert use of these artefacts for the overall success of a software product. Effective social interactions are essential since, as their findings suggest, there are no built-in mechanisms in the physical artefacts or the notation that support the avoidance of errors or the inconsistent use of physical artefacts or notation. As Sharp et al. say:

"Notation does not exist in isolation: it is situated in the reality of a social setting and stands in a reflexive relationship to that reality, i.e. the notation creates and sustains that reality and is created and sustained by it."

The idea of Storycards and Cardwalls is to support the human activity of software creation. We must always try to understand the social roles and interactions that reflexively situate

the use of the software. However, Agile teams must recognize that it is their own social process that ultimately leads to success. 


\section{Simultaneous Support for all Guidelines}

When the guidelines are viewed as a whole, the implications of fully supporting all of our guidelines in a single digital Cardwall implementation could possible introduce complexity for the end-user interaction. In particular, support for the guidelines in the 'exploring and filtering' theme must be carefully considered so as to avoid unnecessary interaction complexity. The ultimate goal is a deceptively powerful system, one in which an experienced physical Cardwall user should be able to start using a well designed digital Cardwall without the need to ask "How do I ...?". The interface and interaction should be obvious and feel natural. For new features not possible with a physical Cardwall, like support for the guidelines in the exploring and filtering theme, careful attention to creating awareness is necessary, using a variety of both visual and audio cues, being careful to avoid clutter and excessive distraction. Similar to the importance of constant feedback discussed by Daniel Wigdor in "A Brave NUI World" [68], the support for exploring and filtering, should provide users with the ability to continuously investigate and work with sets of interesting data, but at the same time the system should provide subtle affordances to help maintain the users awareness of where they are and how to return to the detailed general overview.

\subsubsection{Future Work}

It would of course be desirable to implement a digital Cardwall using the guidelines from this thesis. In particular, this would allow us to conduct usability evaluations and ecologically valid user studies. For professional use, any digital Cardwall implementation should support interfacing with existing story management software and issue trackers tools like JIRA.

\subsection{Contributions}

This main contributions of this thesis were:

- A field study of digital Cardwall use with professional Agile teams.

- Identification of design guidelines for improving digital Cardwalls.

- A survey of interaction design possibilities for the implementation of a digital Cardwall using large multi-touch displays. 


\section{Bibliography}

[1] Agile Alliance. Agile alliance 2011. Web, Feb 2014 http://agile2011. agilealliance.org/.

[2] Caroline Appert, Olivier Chapuis, and Emmanuel Pietriga. High-precision magnification lenses. In Proceedings of the 28th international conference on Human factors in computing systems, CHI '10, pages 273-282, New York, NY, USA, 2010. ACM.

[3] Atlassian. Atlassian. Web, Feb 2014. https://www.atlassian.com/company.

[4] Atlassian. Greenhopper. Web, Feb 2014. https://confluence.atlassian.com/ display/AGILE/GreenHopper+6.0+Release+Notes.

[5] Atlassian. Jira. Web, Feb 2014. https://www.atlassian.com/software/jira.

[6] Atlassian. Jira query language. Web, Feb 2014. https://confluence.atlassian.com/ display/JIRA/Advanced+Searching\#AdvancedSearching-FieldsReference.

[7] Amartya Banerjee, Jesse Burstyn, Audrey Girouard, and Roel Vertegaal. Pointable: an in-air pointing technique to manipulate out-of-reach targets on tabletops. In Proceedings of the ACM International Conference on Interactive Tabletops and Surfaces, ITS '11, pages 11-20, New York, NY, USA, 2011. ACM.

[8] K. Beck. Extreme Programming Explained. Addison-Wesley Professional, 1999.

[9] Hrvoje Benko, Andrew D. Wilson, and Patrick Baudisch. Precise selection techniques for multi-touch screens. In Proceedings of the SIGCHI conference on Human Factors in computing systems, CHI '06, pages 1263-1272, New York, NY, USA, 2006. ACM.

[10] H.R. Bernard. Research Methods in Anthropology. AltaMira Press, 2011.

[11] Eric A. Bier, Maureen C. Stone, Ken Pier, William Buxton, and Tony D. DeRose. Toolglass and magic lenses: the see-through interface. In Proceedings of the 20th annual conference on Computer graphics and interactive techniques, SIGGRAPH '93, pages 73-80, New York, NY, USA, 1993. ACM.

[12] U.M. Borghoff and J.H. Schlichter. Computer-Supported Cooperative Work: Introduction to Distributed Applications. Springer, 2000.

[13] Florence Chabanois. Tools used for agile projects: the survey's results. Web, Mar 2014. http://www.devsnotebook.fr/index.php?post/ Tools-used-for-agile-projects-\%3A-the-survey-results-! 
[14] Citrix. Easy web conferencing and online meeting tools. work with anyone, anywhere. Web, May 2014. http://www.gotomeeting.co.uk/.

[15] A. Cockburn. Agile Software Development: The Cooperative Game, Second Edition. Addison-Wesley Professional, 2006.

[16] Andy Cockburn, Amy Karlson, and Benjamin B. Bederson. A review of overview + detail, zooming, and focus+context interfaces. ACM Comput. Surv., 41(1):2:1-2:31, jan 2009.

[17] M. Cohn. User Stories Applied: For Agile Software Development. Addison-Wesley Professional, 2004.

[18] J. Corbin and A. Strauss. Basics of Qualitative Research: Techniques and Procedures for Developing Grounded Theory. SAGE Publications, 2008.

[19] Software Craftsmanship. Software craftsmanship. Web, Feb 2014. http://manifesto. softwarecraftsmanship.org/.

[20] R. Davies. The power of stories. In The Power of Stories. XP 2001, Sardinia, Springer Verlag, 2001.

[21] Rodrigo A. de Almeida, Clément Pillias, Emmanuel Pietriga, and Pierre Cubaud. Looking behind bezels: French windows for wall displays. In Proceedings of the International Working Conference on Advanced Visual Interfaces, AVI '12, pages 124-131, New York, NY, USA, 2012. ACM.

[22] Digital vision touch, from smart technologies. http://www.smarttech.com/us/About+ SMART/About+SMART/Newsroom/Media+releases/Releases+by+year/2009+media+ releases/2009/June+17+2009c, December 2010 .

[23] Don't Click-it. Understand it don't click it. Web, March 2012. dontclick.it.

[24] Alex Endert, Lauren Bradel, Jessica Zeitz, Christopher Andrews, and Chris North. Designing large high-resolution display workspaces. In Proceedings of the International Working Conference on Advanced Visual Interfaces, AVI '12, pages 58-65, New York, NY, USA, 2012. ACM.

[25] Nigel Fielding and Raymond M. Lee. Computer analysis and qualitative research, chapter 2, pages 21-55. SAGE Publications, 1998.

[26] Andrea Fontana and James H. Frey. Interviewing: The Art of Science, pages 361-376. SAGE Publications, 1994.

[27] Frustrated total internal reflection, from from nui group. http://wiki.nuigroup.com/ FTIR, December 2010. 
[28] T.R.G. Green and M. Petre. Usability analysis of visual programming environments: A 'cognitive dimensions' framework. Journal of Visual Languages and Computing, 7(2):131 - 174, 1996.

[29] Jaber F. Gubrium and Andrea Sankar. Qualitative methods in aging research, volume 168, chapter 12, pages 189-210. SAGE Publications, 1994.

[30] Jeff Huang, Ryen W. White, and Susan Dumais. No clicks, no problem: using cursor movements to understand and improve search. In Proceedings of the 2011 annual conference on Human factors in computing systems, CHI '11, pages 1225-1234, New York, NY, USA, 2011. ACM.

[31] Jörn Hurtienne, Christian Stöíel, Christine Sturm, Alexander Maus, Matthias Rötting, Patrick Langdon, and John Clarkson. Physical gestures for abstract concepts: Inclusive design with primary metaphors. Interact. Comput., 22:475-484, November 2010.

[32] Ratko Jagodic. Collaborative Interaction and Display Space Organization in Large Highresolution Environments. PhD thesis, University of Illinois at Chicago, Chicago, IL, USA, 2011. AAI3551257.

[33] Ratko Jagodic, Luc Renambot, Andrew Johnson, Jason Leigh, and Sachin Deshpande. Enabling multi-user interaction in large high-resolution distributed environments. Future Gener. Comput. Syst., 27(7):914-923, July 2011.

[34] M.R. Jakobsen, Y. Sahlemariam Haile, S. Knudsen, and K. Hornbaek. Information visualization and proxemics: Design opportunities and empirical findings. Visualization and Computer Graphics, IEEE Transactions on, 19(12):2386-2395, Dec 2013.

[35] R. Jeffries. Essential XP: Card, conversation, and confirmation. XP Magazine, August 2001.

[36] Dietrich Kammer, Jan Wojdziak, Mandy Keck, Rainer Groh, and Severin Taranko. Towards a formalization of multi-touch gestures. In ACM International Conference on Interactive Tabletops and Surfaces, ITS '10, pages 49-58, New York, NY, USA, 2010. ACM.

[37] Dominik P. Käser, Maneesh Agrawala, and Mark Pauly. Fingerglass: efficient multiscale interaction on multitouch screens. In Proceedings of the 2011 annual conference on Human factors in computing systems, CHI '11, pages 1601-1610, New York, NY, USA, 2011. ACM.

[38] Azam Khan, Justin Matejka, George Fitzmaurice, Gord Kurtenbach, Nicolas Burtnyk, and Bill Buxton. Toward the digital design studio: Large display explorations. HumanComputer Interaction, 24(1-2):9-47, 2009. 
[39] Sungahn Ko, KyungTae Kim, Tejas Kulkarni, and Niklas Elmqvist. Applying mobile device soft keyboards to collaborative multitouch tabletop displays: design and evaluation. In Proceedings of the ACM International Conference on Interactive Tabletops and Surfaces, ITS '11, pages 130-139, New York, NY, USA, 2011. ACM.

[40] Bongshin Lee, Petra. Isenberg, Nathalie Henry Riche, and Sheelagh Carpendale. Beyond mouse and keyboard: Expanding design considerations for information visualization interactions. Transactions on Visualization and Computer Graphics, IEEE, 18(12), 2012.

[41] John Lofland. Analyzing social settings: a guide to qualitative observation and analysis, chapter 5, pages 81-116. Wadsworth/Thomson Learning, 4th edition, 2006.

[42] Nicolai Marquardt, Ricardo Jota, Saul Greenberg, and JoaquimA. Jorge. The continuous interaction space: Interaction techniques unifying touch and gesture on and above a digital surface. In Pedro Campos, Nicholas Graham, Joaquim Jorge, Nuno Nunes, Philippe Palanque, and Marco Winckler, editors, Human-Computer Interaction - INTERACT 2011, volume 6948 of Lecture Notes in Computer Science, pages 461-476. Springer Berlin Heidelberg, 2011.

[43] Nicolai Marquardt, Johannes Kiemer, David Ledo, Sebastian Boring, and Saul Greenberg. Designing user, hand, and handpart-aware tabletop interactions with the touchid toolkit. In Proceedings of the ACM International Conference on Interactive Tabletops and Surfaces, ITS '11, pages 21-30, New York, NY, USA, 2011. ACM.

[44] Christopher McAdam and Stephen Brewster. Using mobile phones to interact with tabletop computers. In Proceedings of the ACM International Conference on Interactive Tabletops and Surfaces, ITS '11, pages 232-241, New York, NY, USA, 2011. ACM.

[45] Microsoft. Group video calling. Web, May 2014. http://www. skype.com/en/.

[46] R. Morgan. Distributed agile planner: A card-based planning environment for agile teams. Master's thesis, University of Calgary, Department of Computer Science, 2008.

[47] Tomer Moscovich. Contact area interaction with sliding widgets. In Proceedings of the 22nd annual ACM symposium on User interface software and technology, UIST'09, pages 13-22, New York, NY, USA, 2009. ACM.

[48] Adiyan Mujibiya, Takashi Miyaki, and Jun Rekimoto. Anywhere touchtyping: text input on arbitrary surface using depth sensing. In Adjunct proceedings of the 23nd annual ACM symposium on User interface software and technology, UIST '10, pages 443-444, New York, NY, USA, 2010. ACM.

[49] Taichi Murase, Atsunori Moteki, Noriaki Ozawa, Nobuyuki Hara, Takehiro Nakai, and Katsuhito Fujimoto. Gesture keyboard requiring only one camera. In Proceedings of the 24th annual ACM symposium adjunct on User interface software and technology, UIST '11 Adjunct, pages 9-10, New York, NY, USA, 2011. ACM. 
[50] Dmitry Pyryeskin, Mark Hancock, and Jesse Hoey. Extending interactions into hoverspace using reflected light. In Proceedings of the ACM International Conference on Interactive Tabletops and Surfaces, ITS '11, pages 262-263, New York, NY, USA, 2011. ACM.

[51] Herbert J. Rubin and Irene Rubin. Qualitative interviewing: the art of hearing data, chapter 5, pages 93-121. Sage Publications, 1995.

[52] Jaime Ruiz, Yang Li, and Edward Lank. User-defined motion gestures for mobile interaction. In Proceedings of the 2011 annual conference on Human factors in computing systems, CHI '11, pages 197-206, New York, NY, USA, 2011. ACM.

[53] Roger Sanjek. Fieldnotes: The makings of anthropology. Journal of the History of the Behavioral Sciences, 28(3):274-276, 1990.

[54] Dominik Schmidt, Ming Ki Chong, and Hans Gellersen. Handsdown: Hand-contourbased user identification for interactive surfaces. In Proceedings of the 6th Nordic Conference on Human-Computer Interaction: Extending Boundaries, NordiCHI '10, pages 432-441, New York, NY, USA, 2010. ACM.

[55] Dominik Schmidt, Ming Ki Chong, and Hans Gellersen. Idlenses: dynamic personal areas on shared surfaces. In ACM International Conference on Interactive Tabletops and Surfaces, ITS '10, pages 131-134, New York, NY, USA, 2010. ACM.

[56] Sebastian Schmidt, Miguel A. Nacenta, Raimund Dachselt, and Sheelagh Carpendale. A set of multi-touch graph interaction techniques. In ACM International Conference on Interactive Tabletops and Surfaces, ITS '10, pages 113-116, New York, NY, USA, 2010. ACM.

[57] K. Schwaber and M. Beedle. Agile Software Development with Scrum (Series in Agile Software Development). Prentice Hall, 2001.

[58] Stacey D. Scott, Karen D. Grant, and Regan L. Mandryk. System guidelines for colocated, collaborative work on a tabletop display. In Proceedings of the Eighth Conference on European Conference on Computer Supported Cooperative Work, ECSCW'03, pages 159-178, Norwell, MA, USA, 2003. Kluwer Academic Publishers.

[59] Stacey D. Scott, Karen D. Grant, and Regan L. Mandryk. System guidelines for colocated, collaborative work on a tabletop display. In Proceedings of the eighth conference on European Conference on Computer Supported Cooperative Work, ECSCW'03, pages 159-178, Norwell, MA, USA, 2003. Kluwer Academic Publishers.

[60] H. Sharp, H. Robinson, and M. Petre. The role of physical artefacts in agile software development. The Interdisciplinary Journal of Human-Computer Interaction, 21, 2009. issues 1-2. 
[61] Ben Shneiderman. The eyes have it: A task by data type taxonomy for information visualizations. In Proceedings of the 1996 IEEE Symposium on Visual Languages, VL '96, pages 336-, Washington, DC, USA, 1996. IEEE Computer Society.

[62] Garth Shoemaker, Takayuki Tsukitani, Yoshifumi Kitamura, and Kellogg S. Booth. Whole body large wall display interfaces. In Proceedings of the 28th of the international conference extended abstracts on Human factors in computing systems, CHI EA '10, pages 4809-4812, New York, NY, USA, 2010. ACM.

[63] Jonathan A Smith. Rethinking methods in psychology, chapter 2, pages 9-26. Thousand Oaks, 1995.

[64] Fog Creek Software. Trello. Web, Feb 2014. https://trello.com/.

[65] Stephen Voida, Matthew Tobiasz, Julie Stromer, Petra Isenberg, and Sheelagh Carpendale. Getting practical with interactive tabletop displays: designing for dense data, "fat fingers," diverse interactions, and face-to-face collaboration. In Proceedings of the ACM International Conference on Interactive tabletops and surfaces, ITS '09, pages 109-116, New York, NY, 2009. ACM.

[66] X. Wang. Using digital tabletops to support agile project planning. Master's thesis, University of Calgary, Department of Computer Science, Calgary, Alberta, Canada, September 2009.

[67] S. Weber, Y. Ghanam, X. Wang, and F. Maurer. Apdt- an agile planning tool for digital tabletops. In Proceedings 9th International Conference on Agile Processes and eXtreme Programming in Software Engineering (XP2008), 2008.

[68] Daniel Wigdor and Dennis Wixon. Brave NUI World: Designing natural user interfaces for touch and gesture. Morgan Kaufmann Publishers, San Francisco, CA, 1st edition, 2011.

[69] Andrew D. Wilson. Using a depth camera as a touch sensor. In ACM International Conference on Interactive Tabletops and Surfaces, ITS '10, pages 69-72, New York, NY, USA, 2010. ACM.

[70] Jacob O. Wobbrock, Meredith Ringel Morris, and Andrew D. Wilson. User-defined gestures for surface computing. In Proceedings of the SIGCHI Conference on Human Factors in Computing Systems, CHI '09, pages 1083-1092, New York, NY, USA, 2009. ACM.

[71] B. Yost and C. North. The perceptual scalability of visualization. Visualization and Computer Graphics, IEEE Transactions on, 12(5):837-844, Sept 2006. 
Appendix A Table of Quotes 


\begin{tabular}{|c|c|c|}
\hline & Experienced & Inexperienced \\
\hline Traceability & $\begin{array}{l}\text { "if we need to reference a story we might look } \\
\text { back at it, but very infrequently do we go } \\
\text { back to old stories ... you look at the software } \\
\text { to see what it actually does." (John) }\end{array}$ & $\begin{array}{l}\text { "Once it goes to QA, if they find one } \\
\text { [a bug], they would just put the card } \\
\text { back up into In Progress (and put) } \\
\text { the bug-sticky on there [the card]. ... } \\
\text { They would also actually log the } \\
\text { bug on an online bug tracker, just } \\
\text { so that those are all centralized ... } \\
\text { Sometimes it [the bug] would be } \\
\text { created as a story, if it was a } \\
\text { significant bug, like enough time } \\
\text { would be needed, or if it was just } \\
\text { high enough priority. But sometimes } \\
\text { it would just be like: we need } \\
\text { someone to get this done now, so, } \\
\text { (someone's) got to take care of } \\
\text { it."(Keith) }\end{array}$ \\
\hline $\begin{array}{l}\text { Awareness- } \\
\text { external to the } \\
\text { team }\end{array}$ & $\begin{array}{l}\text { "My email box-my inbox is sacred. I don't } \\
\text { want to use email to notify me that stuff is } \\
\text { happening [a story has been changed or it } \\
\text { has changed state]. I feel it's the wrong } \\
\text { medium. So I just check the board. The } \\
\text { physical board is awesome because it's just } \\
\text { there. You just look up at it and it's there, } \\
\text { right? I mean, that's the point-you don't } \\
\text { have to be notified, you can just look." (John) } \\
\text {... stories mid-iteration don't happen often. } \\
\text { That's sort of out of cycle, that's sort of the } \\
\text { exception. So I'm not worried about that. If } \\
\text { that were to happen a lot, I (would) want to } \\
\text { be notified. (John) } \\
\text { The customer uses it [the cardwall]. People } \\
\text { are watching projects. So if I'm working on } \\
\text { multiple projects, even if someone else is } \\
\text { leading it, I'll check in. So I mean, our CEO will } \\
\text { check the wall occasionally, I think, just to see } \\
\text { where an iteration's at. Yeah, I mean, it's great } \\
\text { transparency and visibility, so, (that's) always } \\
\text { there. (John) } \\
\text { "At the daily standups [we use the Cardwall], } \\
\text { I'll bring a laptop and the entire team will } \\
\text { look at the Cardwall so we can have a } \\
\text { reference point to have discussions. Myself, as } \\
\text { a manager, I'm constantly looking at the } \\
\text { Cardwall throughout the day" (Michael) }\end{array}$ & $\begin{array}{l}\text { We ended up having like a red, } \\
\text { yellow or green section. Where } \\
\text { green was used to represent what } \\
\text { we were pretty sure we could finish } \\
\text { in one iteration. Yellow represented } \\
\text { [stories] that we think we still can } \\
\text { [get done], but it's possible it won't } \\
\text { get done. And then, red [stories that } \\
\text { were not likely to get to]. So we had } \\
\text { enough [stories] so that we should } \\
\text { have extras. So if we are done with } \\
\text { all the green [stories]-I don't think } \\
\text { we've ever (gone) past the red- } \\
\text { usually we have a pretty healthy } \\
\text { amount. So I guess that's kind of a } \\
\text { backlog, (there)." (Keith) } \\
\text { [We like to] have a full view of } \\
\text { what's in progress (Keith) } \\
\text { We usually met around it [the } \\
\text { physical Cardwall]. So, you'd take a } \\
\text { look at what's up and what we have } \\
\text { [started or finished] (Keith) } \\
\text { We had sticky notes ... [describing] } \\
\text { the tasks for the story. So we'd split } \\
\text { that up a little bit and then put the } \\
\text { stickies on the wall, in whatever } \\
\text { place it is, (then I could take the } \\
\text { card).... and then put the stickies in }\end{array}$ \\
\hline
\end{tabular}




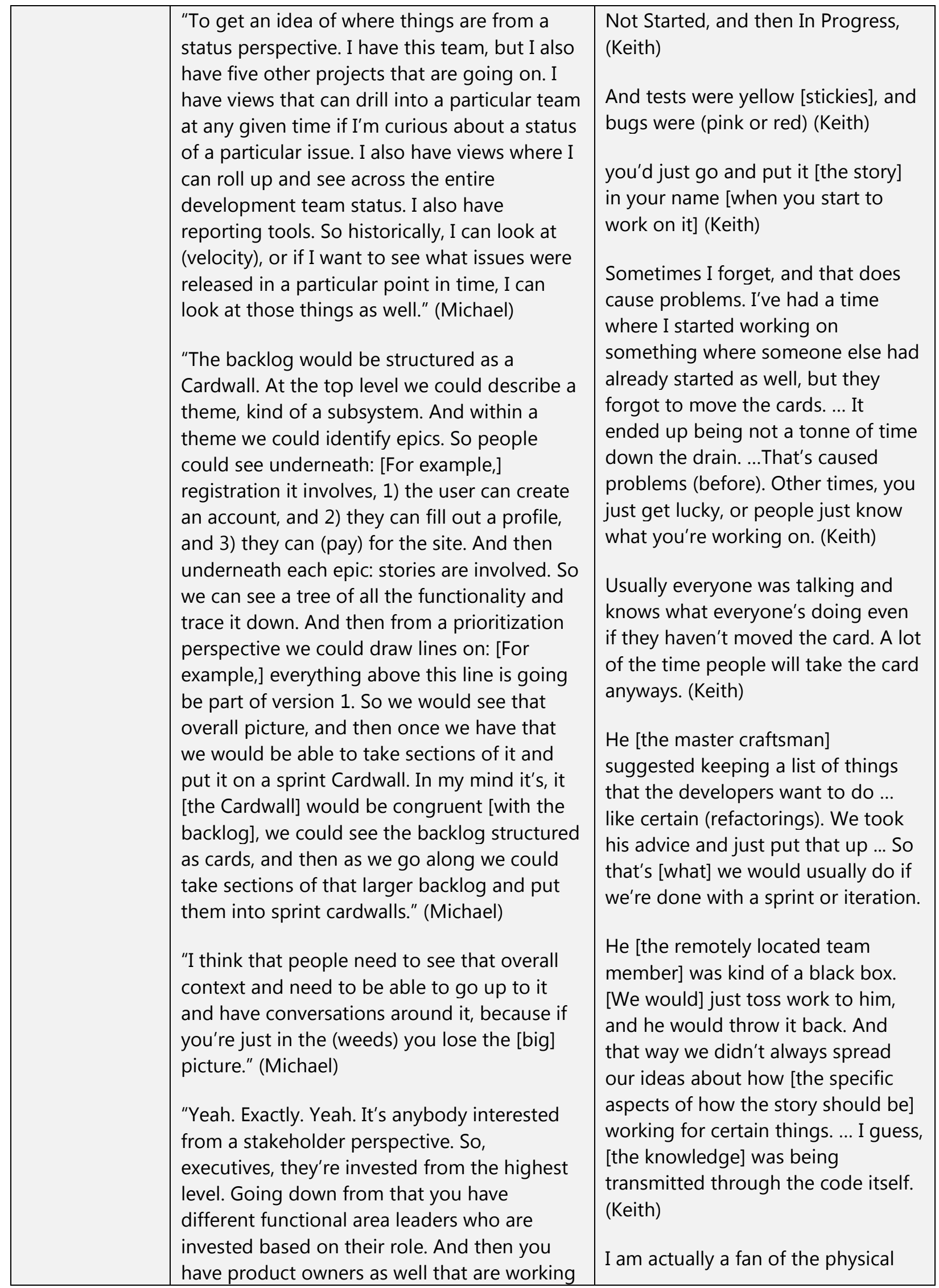




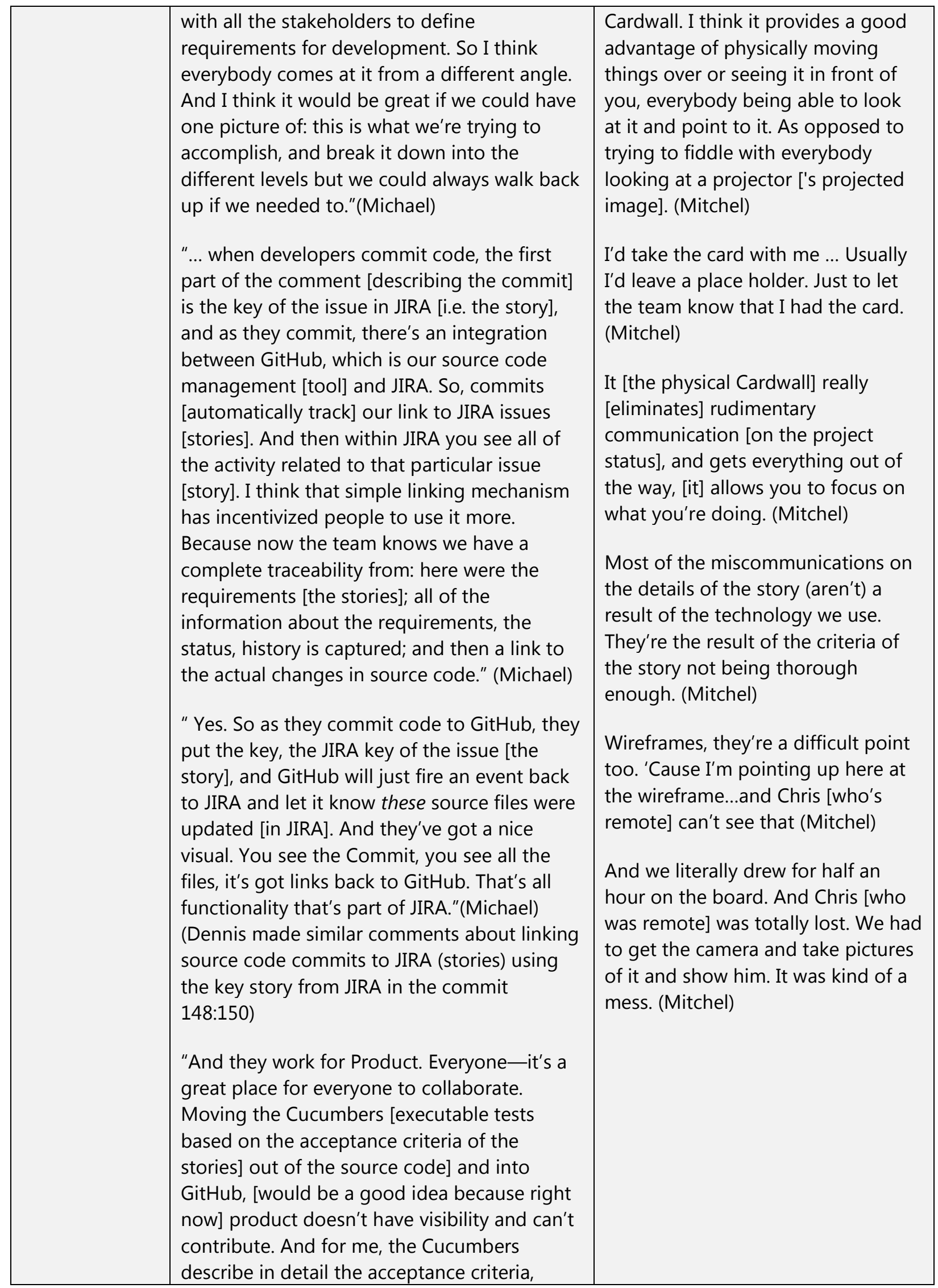




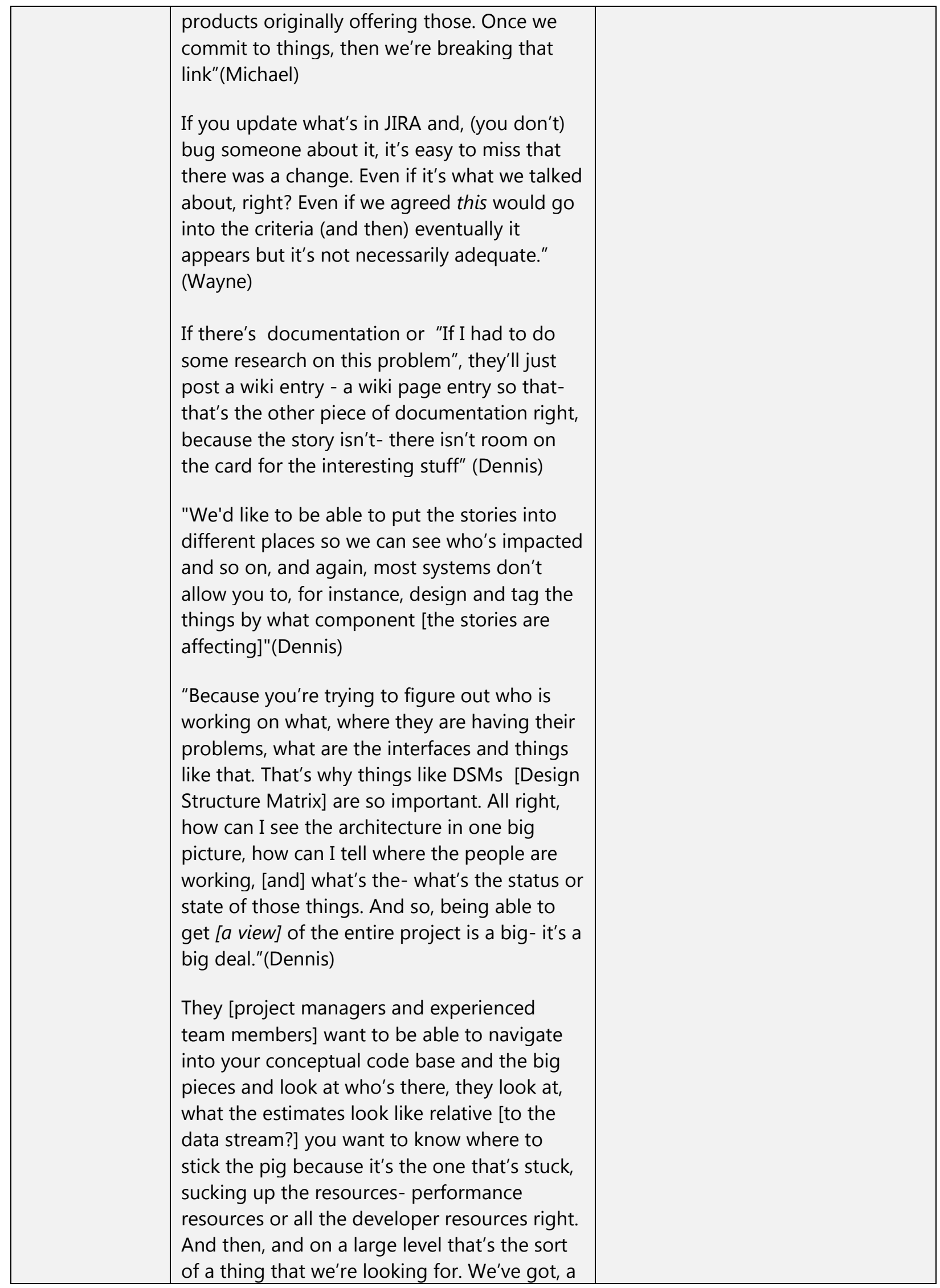




\begin{tabular}{|c|c|c|}
\hline & $\begin{array}{l}\text { company with, } 15 \text { offices all over the world, } \\
\text { [we need] some idea every day of what's } \\
\text { going on. "(Dennis) } \\
\text { People can easily see the progress of a } \\
\text { project by looking at a Cardwall, different } \\
\text { stakeholders will use the Cardwalls to get a } \\
\text { quick status update. } \\
\text { There is a delicate balance wrt to the issue of } \\
\text { notifications. }\end{array}$ & \\
\hline $\begin{array}{l}\text { Awareness - } \\
\text { Internal to the } \\
\text { team }\end{array}$ & & \\
\hline Story grouping & $\begin{array}{l}\text { "and I think the real thing is to be able to } \\
\text { easily break things [down]- to be able to } \\
\text { easily decompose things into stories, group } \\
\text { them together, and then produce information } \\
\text { by just grouping them into story, you know, } \\
\text { just take them and say, "here, I want all these I } \\
\text { want to throw them in a bucket and I want to } \\
\text { ask [some guy? Some body?] how long will } \\
\text { this take you to get better, how much defects } \\
\text { are there, who's working on it, are we behind } \\
\text {... all those sorts of things are things you'd like } \\
\text { to [be able to] do." (Dennis) } \\
\text { "we will actually label something like a } \\
\text { component so we'll have the component ['s] } \\
\text { stories together"(Dennis) } \\
\text { "So, we call those [grouped stories] epics, } \\
\text { often. For estimation purposes, when you } \\
\text { don't want to get too fine a grain of detail, } \\
\text { we'll create an epic." (John) } \\
\text { The other way [of] grouping would be to tie } \\
\text { these stories together as a release maybe, or } \\
\text { a feature set, or even tying it back to an epic } \\
\text { after the fact." (John) } \\
\text { So I think you're asking [about] grouping } \\
\text { stories maybe like tagging them all as...I don't } \\
\text { know...this type of feature, right? ... So ... we } \\
\text { colour-code our stories." (John) } \\
\text { "And there may be different teams working }\end{array}$ & $\begin{array}{l}\text { [we have used colours] for the } \\
\text { stickies. But that included [special } \\
\text { colours for] bugs and tests. It was } \\
\text { really just tests and bugs. And tests } \\
\text { were yellow, and bugs were (pink or } \\
\text { red). (Keith) }\end{array}$ \\
\hline
\end{tabular}




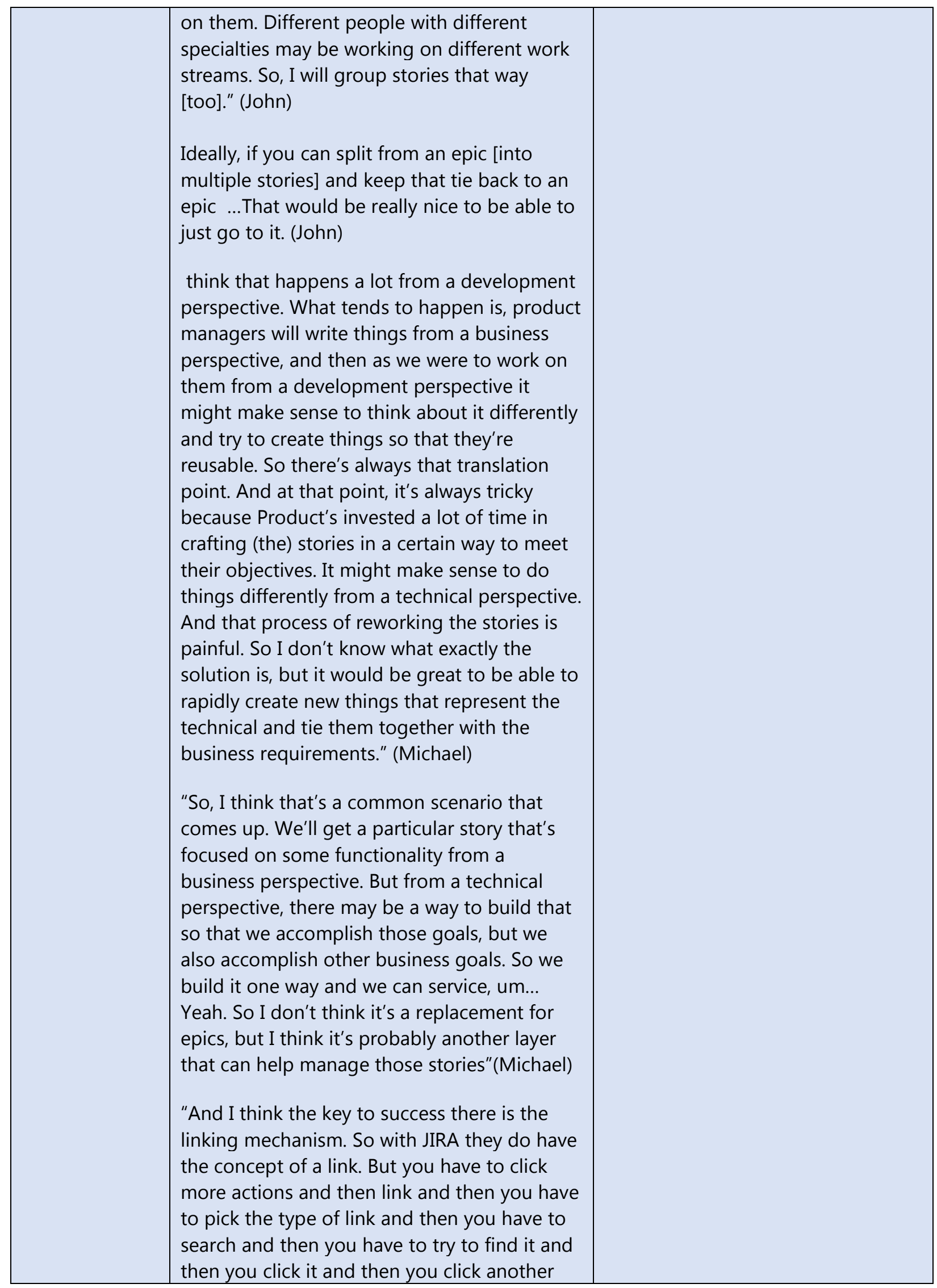




\begin{tabular}{|c|c|c|}
\hline & $\begin{array}{l}\text { thing-and it's too much. It's way to hard to } \\
\text { do, so nobody does it. Or, it's done but it's } \\
\text { not done consistently." (Michael) } \\
\text { Participants identified five different grouping } \\
\text { mechanisms. }\end{array}$ & \\
\hline Communication & $\begin{array}{l}\text { "every week you'll hear: "I thought what we } \\
\text { talked about was..." And, it's on us to read the } \\
\text { AC better. And it's also on management to } \\
\text { notify us, send us emails when they actually } \\
\text { get around to updating what's in JIRA. So if } \\
\text { you update what's in JIRA and, (you don't), } \\
\text { bug someone about it, it's easy to miss that } \\
\text { there was a change. Even if it's what we talked } \\
\text { about, right? Even if we agreed this would go } \\
\text { into the criteria. Eventually it appears but it's } \\
\text { not necessarily adequate." (Wayne) } \\
\text { "[Viewing digital Cardwalls during daily } \\
\text { standups on a shared display] the entire team } \\
\text { will look at the Cardwall so we can have a } \\
\text { reference point to have discussions." } \\
\text { (Michael) } \\
\text { "[With regard to how detailed a story should } \\
\text { be] ... we like to favour verbal conversations. } \\
\text { So, I think: enough to describe what we're } \\
\text { trying to accomplish, but not too much as to } \\
\text { be, you know, some big document that } \\
\text { nobody's gonna read or possibly misinterpret. } \\
\text { I think verbal communication is much more } \\
\text { effective when it comes to specific details." } \\
\text { (Michael) } \\
\text { "[how strong communication between teams } \\
\text { helps refine and prioritize stories] We also } \\
\text { have a process where, before (IPMs), Product } \\
\text { Development and QA will get together and } \\
\text { review stories that are gonna be considered } \\
\text { for the next iteration. So through that we're } \\
\text { collaboratively authoring or refining stories." } \\
\text { (Michael) }\end{array}$ & $\begin{array}{l}\text { Other times, I mean, you just get } \\
\text { lucky, or people just know what } \\
\text { you're working on ... by people } \\
\text { talking [about the stories they're } \\
\text { working on]. (Keith) } \\
\text { If I couldn't talk to Jay about a story } \\
\text { throughout the week, I would be in } \\
\text { a lot of trouble. I need constant } \\
\text { clarification... Interviewer: Do you } \\
\text { think that if he wasn't actually on } \\
\text { site, would you be able to have that } \\
\text { same type of communication?] } \\
\text { That's a question I've been asking } \\
\text { myself too because we've been } \\
\text { considering working remotely out of } \\
\text { our office at Octagon. And I have } \\
\text { been hesitant to make that move } \\
\text { because I feel like I would lose that } \\
\text { communication with Jay, which is } \\
\text { very valuable. So I don't think so. I } \\
\text { think that being in the same office } \\
\text { offers a massive benefit. }\end{array}$ \\
\hline
\end{tabular}


Appendix B Ethics Approval 


\section{Carleton}

U N I V E R S I Y

Canada's Capital University
School of Computer Science

5302 Herzberg Laboratories

1125 Colonel By Drive

Ottawa, ON K1S 5B6 Canada

Tel: 613-520-4333

Fax: 613-520-4334

Web site: www.scs.carleton.ca

\section{Appendix 2: Semi-Structured Interview Script}

The following questions are a general guideline for the interviews. The point of the semistructured interview is to acquire insight into individual team member's roles and practices with respect to user stories and their management via a physical cardwall or software and to try and find commonalities that could lead to a theory about the software development processes. The interviewer will try and explore and delve deeper into some areas depending on the answers given. We are interested in exploring specific data from individual user experiences through all stages of software project.

1. We are interested in any experiences you have had with user stories and their management.

a. How long have you been using user stories to help plan and track your software projects?

b. Have you ever created a user story? Explain.

c. Who creates the user stories? Why?

d. What medium is used for the user stories? Why?

e. How often are the user stories updated? When and Why?

f. Is the creation of a backlog of user stories the first thing your team does when planning a project, release or sprint?

g. Are there stories that don't make it into the backlog? Explain.

h. Are stories ever permanently deleted? Why

i. How is the team made aware of changes in a story?

j. How does the team know when a story is complete?

k. How does the team know when other potential state changes occur like when a story is assigned to a team or developer for implementation?

2. Do all team members physically work in the same location? If no then:

a. How has your team adapted their use of user stories and cardwalls to better support distributed teams?

i. Are there specific aspects of user stories and cardwalls that don't work well with distributed teams?

ii. Are there specific aspects of user stories and cardwalls that are particularly well suited for distributed teams?

b. Can you remember an instance when you had to collaborate with a distributed team member? Explain.

c. Are there some tasks or specific types of meetings where user stories or their management play an important role in the meeting or task and the task or meeting is particularly well suited for collaboration in a distributed environment? 
d. When collaborating are the contribution of the remote team members easily recognized by the other team members.

e. Can you remember a time when you (or someone else) missed something important during a distributed collaborative session because you were not aware of any changes?

3. Do you use a cardwall to manage user stories?
a. Why do use a cardwall?
b. How often?
c. Who participates?
d. What are the roles?
e. What are the goals?
f. What do the columns mean?
g. What do the rows mean?
h. In your experience have you noticed any specific problems that cardwalls resolve?

i. In your experience have you noticed any potential problems created by the use of cardwalls

j. Have you ever used a cardwall in a distributed environment?

$\mathrm{k}$. Is your cardwall on constant display somewhere it can be easily seen by all team members?

I. Is it a physical one? If yes.

i. Have you ever considered using software to manage your user stories (or do you use a combination)?

ii. Where is your cardwall located?

iii. Does everyone have equal access to view the wall?

iv. Does everyone have equal access to manipulate stories on the wall?

v. Does you wall represent a specific level of granularity i.e. one sprint or one release?

vi. How is your backlog managed?

vii. Is there ever a need to see more information than what is presented on your wall?

viii. Would more than one wall be more useful?

ix. Do you remove cards from the wall?

$x$. Can you create new stories and place them on the wall?

xi. Do you have a wall to manage your backlog?

xii. Are stories temporarily removed from the wall?

1. How does that work?

xiii. Within your organization are there other teams who also use physical cardwalls

1. Are there differences in how distinct teams use the cardwalls?

2. Does your team use customized notations?

xiv. Are there any advantages to using a physical cardwall? Explain?

m. Is your cardwall implemented in software? If yes.

i. How do you use the software?

ii. What are the advantages of the software?

iii. Does it support collaboration? How? 
iv. Does it support distributed collaboration? How?

v. Can software adequately replace or a physical cardwall? Why and How?

n. Does the use of your cardwall during collaboration help support your awareness of:

i. The different potential activities that could be simultaneously occurring during a collaborative session

ii. Who was the initiator of a particular activity?

iii. Ownership of a particular artefact or activity.

iv. Session history.

v. Group contributions that lead to an advancement in the overall understanding of the task or activity.

4. Do you use a combination of software and physical cardwall? Why?

5. Do you use software to help manage you user stories that does not have anything similar to a cardwall?

6. Can you describe your most recent planning meeting?

a. How long did it last?

i. Was this a typical meeting?

b. Where was it they held? Why there?

i. Are they always in same place?

c. Who else participates?

d. What are the goals?

i. Do they change?

e. Do you have a specific role in the planning meeting? Explain.

f. Are there specific roles that team members adopt during planning meetings?

i. Does one person act as a scribe? Why

g. Does your team's use of user stories and cardwalls support these roles? How? 
Carleton

U N I V E R S I T Y

Canada's Capital University
School of Computer Science

5302 Herzberg Laboratories

1125 Colonel By Drive

Ottawa, ON K1S 5B6 Canada

Tel: $613-520-4333$

Fax: 613-520-4334

Web site: www.scs.carleton.ca

\section{Appendix 3: Informed Consent for the Interview}

The purpose of an informed consent is to ensure that you understand the purpose of our study and the nature of your involvement. I am glad to answer any questions not answered by this document. Please contact:

Judith Brown, principal researcher and PostDoctoral Fellow, School of Computer Science

Carleton University

1125 Colonel By Drive

Ottawa, Ontario K1S 5B6

Tel: $613-520-2600 \times 1987$

Email: mmjbrown@connect.carleton.ca
Stevenson Gossage, student researcher, School of Computer Science

Carleton University

1125 Colonel By Drive

Ottawa, Ontario K1S 5B6

Tel: $613-520-2600 \times 1987$

Email: sgossage@connect.carleton.ca

Any other concerns, questions or issues can be directed to:

Professor Antonio Gualtieri, Chair Research

Ethics Board

Carleton University Research Office

1125 Colonel By Drive

Ottawa, Ontario K1S 5B6

Tel: 613-520-2517

Email: ethics@carleton.ca

Task Requirements: In this study you will be asked to participate in one semi-structured interview lasting half an hour to one hour. During the interview you will be asked about your team's use of cardwalls and user stories. A semi-structured interview means that each interview will have broad categories of interest like cardwalls and user stories and the specific questions will be based on the interview script provided, however the process is an explorative one and will therefore vary from the provided script based on individual responses.

Duration and locale: The interview will last from one half hour to one hour, and can be conducted at the location of your choice.

Data: The audio from the interviews will be recorded and transcribed. These data will be destroyed upon completion of the analysis or at the latest by 31/Aug/2014. 
Specific Right to Withdraw: You have the right to withdraw at any time during your interview and you may decline to answer any questions in the interview without penalty. You also have the right to drop out of the study at any time up to one month after the date of your interview. If, in the future, you decide that you do not want to be involved in the study, you may contact Judith Brown (613-254-8955

mmjbrown@connect.carleton.ca) or Stevenson Gossage (sgossage@connect.carleton.ca, 613-520-2600 X 1987) to have the information you provided removed. You also have a right to take a break at any time. If you do choose to withdraw from the study your interview data will be destroyed immediately.

I am aware that the reason for informed consent is to make sure that I understand why the study is being done, how I will be involved and what my rights are. I have been told that I will be interviewed in this study. I have seen the script that the interview will be based on. I understand that the researcher needs information about user stories and cardwall usage in order to understand the practices of agile teams and their use of user stories and cardwalls and that this is the main purpose of my interview. I understand that I do not have to answer any question that makes me feel uncomfortable.

My signature below indicates that I have read the above information and that I agree to take part in this study. It also indicates that I agree to having the interview recorded, understanding that the recording will be destroyed upon completion of the study.

Signature (participant):

Signature (researcher): Date: 
Carleton

U N I V E R S I T Y

Canada's Capital University
School of Computer Science

5302 Herzberg Laboratories

1125 Colonel By Drive

Ottawa, ON K1S 5B6 Canada

Tel: $613-520-4333$

Fax: 613-520-4334

Web site: www.scs.carleton.ca

\section{Appendix 4: Introductory Letter to Team Leader}

The purpose of this letter is to inform you about our desire to have your team participate in our study about cardwalls and user stories. Through the observation of real world teams we hope to gain insight and develop theories about how these artifacts are used. This knowledge will inform the design and implementation of our own digital cardwall. We would like to observe your planning meetings while interfering as little as possible with your work. On occasion we may ask for permission to take a photograph of cardwalls or stories.

We are interested in the process and not the product being developed. We will not disclose any sensitive information. We would be happy to sign any non-disclosure or intellectual property agreement you may feel is necessary. Your company, the product and the participants will never be refereed to using anything but pseudonyms in any paper or report we may write. Photographs, taken with your permission, would include no product details or photos of individuals. Any information identifying your product or organization would be removed.

We ask to arrange a short preliminary meeting to introduce myself and my team, explain the study and the underlying methods and finally to get a signed consent form from each team member. Each team member must fill out and sign the form in our presence. We designed the form such that team members can choose to opt in or out of the study in an anonymous fashion and we will only review the forms in private after the conclusion of this preliminary meeting. If any team member prefers not to participate then we will not use your team as part of our study. Information about participation or lack thereof will not be shared with you or any other team member.

We would like to thank you in advance for your initial interest in our study and we appreciate the opportunity to work with and your team.

For any questions or concerns please contact:

Judith Brown, principal researcher and PostDoctoral Fellow,

School of Computer Science

Carleton University

1125 Colonel By Drive

Ottawa, Ontario K1S 5B6

Tel: $613-520-2600 \times 1987$

Email: mmjbrown@connect.carleton.ca

Faculty \& Staff Research Protocol Form July 2010
Stevenson Gossage, student researcher, School of Computer Science

Carleton University

1125 Colonel By Drive

Ottawa, Ontario K1S 5B6

Tel: $613-520-2600 \times 1987$

Email: sgossage@connect.carleton.ca 
Any other concerns, questions or issues can be directed to:

Professor Antonio Gualtieri, Chair Research

Ethics Board

Carleton University Research Office

1125 Colonel By Drive

Ottawa, Ontario K1S 5B6

Tel: 613-520-2517

Email: ethics@carleton.ca 
Carleton

U N I V E R S I T Y

Canada's Capital University
School of Computer Science

5302 Herzberg Laboratories

1125 Colonel By Drive

Ottawa, ON K1S 5B6 Canada

Tel: $613-520-4333$

Fax: 613-520-4334

Web site: www.scs.carleton.ca

\section{Appendix 5: Informed Consent for Observations}

The purpose of this informed consent is to ensure that you understand the nature of your involvement in this part of the study. I am glad to answer any questions not answered by this document. Please contact:

Judith Brown, principal researcher and PostDoctoral Fellow, School of Computer Science

Carleton University

1125 Colonel By Drive

Ottawa, Ontario K1S 5B6

Tel: $613-520-2600 \times 1987$

Email: mmjbrown@connect.carleton.ca
Stevenson Gossage, student researcher, School of Computer Science

Carleton University

1125 Colonel By Drive

Ottawa, Ontario K1S 5B6

Tel: $613-520-2600 \times 1987$

Email: sgossage@connect.carleton.ca

Any other concerns, questions or issues can be directed to:

Professor Antonio Gualtieri, Chair Research

Ethics Board

Carleton University Research Office

1125 Colonel By Drive

Ottawa, Ontario K1S 5B6

Tel: 613-520-2517

Email: ethics@carleton.ca

Task Requirements: This part of the study will require you, as a team member to allow us, the researchers, to observe team planning meetings in which you participate. It will be expected that you conduct your work in your normal manner. I will be taking notes and on occasion may ask permission to take a photograph.

Duration and locale: The observations will last for the duration of the meetings. This will take place in your normal workplace.

Data: The photos and notes will be viewed only by the research team, only for research purposes, and will be stored in a secure location. These data will be destroyed upon completion of the study, at the latest 31/Aug/2014. 
Specific Right to Withdraw: You have the right to withdraw at any time up to one month after the end of the observation period. You may also ask to restrict my observations in any way that suits you.

I am aware that the reason for informed consent is to make sure that I understand why the study is being done and how I will be involved. I have been told that my team will be observed in this study. I understand that the researcher wishes to observe user story and cardwall use to understand how agile teams use user stories and cardwalls and that this is the main purpose of the observation. I understand that I have control over what is observed and that I may drop out of the study at any time up to one month after the end of the observation period, without any consequences.

I understand that under no circumstances will any of this information be used to misrepresent or harm me. I further understand that all names will be removed from data and these data will be pooled to represent all users. As a result, no one will be identifiable or mentioned by name. My signature below indicates that I have read the above information and that I agree to take part in this study.

Signature (subject):

Signature (researcher): Date: 
Carleton

U N I V E S I T Y

Canada's Capital University
School of Computer Science

5302 Herzberg Laboratories

1125 Colonel By Drive

Ottawa, ON K1S 5B6 Canada

Tel: $613-520-4333$

Fax: 613-520-4334

Web site: www.scs.carleton.ca

\section{Appendix 6: Demographic Questionnaire}

The demographic questionnaire provides information about the maturity and experience of the agile team. This type of information is generally very useful in a grounded theory analysis. We are also interested in capturing the roles of individual team members and their background. This is important since agile teams are multidisciplinary and each person plays a different role. Understanding these roles helps us to understand cardwall use and the importance of different aspects of the card wall to the different team members.

\section{General}

1. What is your job title?

2. How many years of experience do you have as a member of an agile team?

3. How many years of post-secondary education do you have?

4. What post-secondary qualifications do you have?

5. Do you consider yourself to be an extrovert or an introvert?

6. How much do you enjoy collaborative work? Answer using a scale of 1 to 10 , where 1 is do not enjoy at all; and 10 is extremely enjoy.

Project-specific

7. What is your role on the agile project team?

8. What are the roles of other individuals on your agile project team?

9. During meetings that involve your team members updating your card wall are there other computing devices in the room? If so, what are these devices?

10. In your experience how effective are the user stories written for your project? Answer using a scale of 1 to 10 , where 1 is not effective at all; and 10 is extremely effective.

11. Do you write or create user stories?

12. What is your primarily involvement with user stories?

13. In your experience how effective is the card wall used by your team? Answer using a scale of 1 to 10 , where 1 is not effective at all; and 10 is extremely effective. 
Carleton

U N I V E R S T Y

Canada's Capital University
School of Computer Science

5302 Herzberg Laboratories

1125 Colonel By Drive

Ottawa, ON K1S 5B6 Canada

Tel: $613-520-4333$

Fax: 613-520-4334

Web site: www.scs.carleton.ca

\section{Appendix 7: Debriefing Form}

The purpose of this study was to learn about the way agile teams use cardwalls and user stories to facilitate project planning. Through the observation of real world teams we hope to gain insight and develop theories about how these artifacts are used. We would also like to understand if there are any less then obvious advantages that these artefacts afford to support the individual roles team members adopt during a software development project. This knowledge will inform the design and implementation of our own multitouch digital cardwall. It is important to note that we are interested in the process and not the product being developed.

For any further questions please contact:

Judith Brown, principal researcher and Postdoctoral fellow, School of Computer Science

Carleton University

1125 Colonel By Drive

Ottawa, Ontario K1S 5B6

Tel: 613-520-2600 X 1987

Email: mmjbrown@connect.carleton.ca
Stevenson Gossage, student researcher, School of Computer Science

Carleton University

1125 Colonel By Drive

Ottawa, Ontario K1S 5B6

Tel: $613-520-2600 \times 1987$

Email: sgossage@connect.carleton.ca

Any other concerns, questions or issues can be directed to:

Professor Antonio Gualtieri, Chair Research

Ethics Board

Carleton University Research Office

1125 Colonel By Drive

Ottawa, Ontario K1S 5B6

Tel: 613-520-2517

Email: ethics@carleton.ca 